\title{
32. CALCAREOUS NANNOFOSSIL BIOSTRATIGRAPHY AND PALEOCLIMATIC INDICES FOR THE LATE QUATERNARY, DEEP SEA DRILLING PROJECT LEG 96, GULF OF MEXICO ${ }^{1}$
}

\author{
R. E. Constans, Chevron USA, Inc. \\ and \\ M. E. Parker, Florida State University²
}

\begin{abstract}
Sediments from holes drilled at 11 sites in the northern Gulf of Mexico during Deep Sea Drilling Project Leg 96 were analyzed for calcareous nannofossil content. All sediments recovered are Holocene and late Pleistocene in age and are within the Emiliania huxleyi Zone. The datum level represented by the lowest stratigraphic occurrence of dominant $E$. huxleyi occurs at two sites (Sites 615 and 619) and can be dated at approximately 84,000 yr. ago at Site 619.

Reworked Cretaceous nannofossils are generally common or abundant and dominate the floral assemblages of the late Wisconsin glacial sediments. When present, indigenous late Quaternary species are rare or few in abundance. Slight increases in the contemporaneous Quaternary component of the floral assemblages can be documented by the use of a calculated in situ/reworked ratio. This ratio, based on the relative abundances of the indigenous Quaternary taxa and reworked taxa, shows potential both for local correlations between drill sites and for correlation with glacio-eustatic fluctuations during the late Pleistocene.
\end{abstract}

\section{INTRODUCTION}

Leg 96 of the Deep Sea Drilling Project (DSDP) was the third cruise of the Glomar Challenger into the Gulf of Mexico, but was the first cruise dedicated to a region of rapid late Pleistocene deposition south of the Mississippi River distributary system. A total of 308 hydraulic piston and rotary cores containing $1302 \mathrm{~m}$ of sediment were taken at 11 sites, 9 on the Mississippi Fan and 2 to the northwest in intraslope basins (Fig. 1).

The majority of the sediment is late Pleistocene in age; only a thin veneer $(<50 \mathrm{~cm})$ of overlying Holocene sediment was recovered at most sites. The horizon marked by the first evolutionary occurrence of Emiliania huxleyi was not penetrated at any of the sites.

As will be demonstrated in this chapter, the typical floral sequence observed in the Mississippi Fan sediments can be characterized by four units. The Holocene (Ericson Zone Z; Ericson and Wollin, 1968) is represented by less than $1 \mathrm{~m}$ of a marly foraminiferal ooze or calcareous clay containing abundant, fair to moderately preserved calcareous nannofossils. Few reworked Cretaceous nannofossils are present in this zone. The underlying terrigenous sand and mud sequences deposited during the late Wisconsin glacial (Ericson Zone Y) are dominated by common to abundant, solution-resistant Late Cretaceous nannofossils. Few indigenous Pleistocene nannofossils are present in this interval. The middle Wisconsin interstadial (Ericson Zone X) contains abundant, moderately preserved Pleistocene species and exhibits a significant reduction in reworked Cretaceous forms. The cool, early Wisconsin glacial stage (Ericson Zone W)

\footnotetext{
${ }^{1}$ Bouma, A. H., Coleman, J. M., Meyer, A. W., et al., Init. Repts. DSDP, 96: Washing. ton (U.S. Govt. Printing Office).

2 Addresses: (Constans) Paleontology Section, Chevron, U.S.A., Inc., 935 Gravier St., New Orleans, LA 70112; (Parker, present address) AMOCO Production Company, P.O. Box 50879, New Orleans, LA 70150 .
}

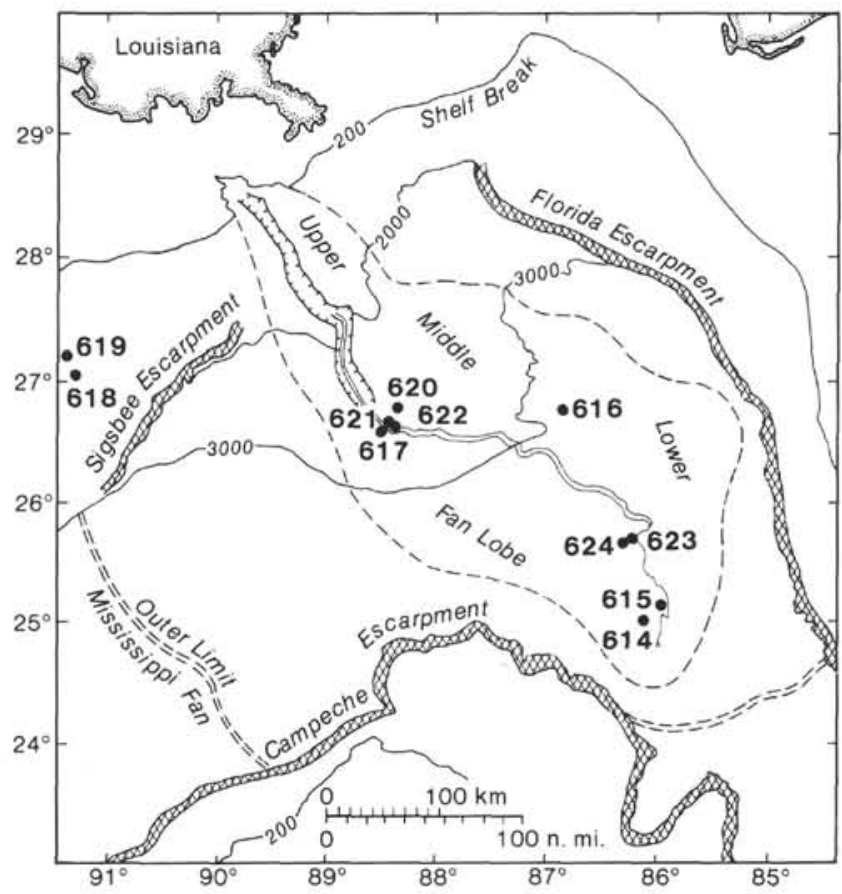

Figure 1. Location map of sites drilled on DSDP Leg 96.

consists of terrigenous clastic sediments dominated by reworked Cretaceous nannofossils. Rare, reworked early Pleistocene, Pliocene, and Miocene species are found throughout all units but are most prevalent in the warmer water intervals.

A similar four-unit floral sequence is encountered in the intraslope basin sites. Greater abundances of indigenous Pleistocene species, however, are present in the Wisconsin glacial intervals.

A number of previous studies have been made of the calcareous nannofossils from Gulf of Mexico sediments. 
Among these are the studies by Bukry and Bramlette (1969) and Hay (1969), which give a preliminary survey of the species recovered from sites drilled during DSDP Leg 1. Although not detailed, the thick Pleistocene section recovered in Hole 1 of DSDP Leg 1 shows a striking resemblance to the sediments recovered on DSDP Leg 96. Ellis et al. (1972) presented a detailed study of the calcareous nannofossils recovered from Site 3 of DSDP Leg 1 in the western Gulf of Mexico. Studies of the nannofossils encountered in DSDP Leg 10 sediments have been made by Hay (1973), Bukry (1973) and by Smith (in Smith and Beard, 1973). The same biostratigraphic framework for the Pleistocene of the Gulf of Mexico used by Smith and Beard in 1973 is presented again in Beard et al. (1982). A calcareous nannofossil biostratigraphy based on a more conventional framework of late Neogene earth history is given by Chen (1978) and Gartner et al. (1983) in their studies of two holes (E67-134 and E67-135) drilled by Shell Oil Company in the De Soto Canyon region of the northeast Gulf of Mexico. The nannofossil floral assemblages from Gulf of Mexico bottom-sediment samples and cores are well documented by Sachs (1970), Sachs and Skinner (1973), and Pierce and Hart (1979).

Paleontologic interpretations of Leg 96 sediments are complicated by extensive dilution from terrigenous detritus resulting from the high sedimentation rates of up to $12 \mathrm{~m} / 1,000$ yr. that are characteristic of the Mississippi Fan (site chapters, this volume; Wetzel and Kohl, this volume). A majority of the section is dominated by reworked Cretaceous taxa. Bukry (1974) showed that the abundance and type of reworked assemblages can be used to interpret the geologic history of somewhat similar sediment sequences in the Black Sea. Everett (1982) demonstrated that estimated counts of indigenous and reworked nannofossil species can be used as an aid in the subsurface interpretation of both ancient and recent Mississippi River deltas. A possible correlation between high sedimentation rates and high amounts of reworked nannofossil species was reported by Ellis and Lohman (1979) in the eastern Mediterranean DSDP Sites 375 and 376.

The main purpose of this chapter is to document the abundance and biostratigraphic distribution of the calcareous nannofossils. No attempt is made to present a complete taxonomic study.

\section{METHODS AND PROCEDURES}

Smear slides of 780 processed samples were examined for the calcareous nannofossil content with a light microscope in both cross-polarized and partial-polarized light. In order to maintain a semiquantitative control for the abundance of nannofossils recorded, the samples were processed in the following manner: $500 \mathrm{mg}$ of unprocessed sample were placed in a 2-fluid-dram bottle and $70 \mathrm{ml}$ of water were added. The sample was ground with a metal stirring rod, shaken, and allowed to settle for $1.5 \mathrm{~min}$. Nannofossil solution was then extracted with a capillary tube placed approximately $1.5 \mathrm{~cm}$ below the surface of the solution. Slides were mounted employing commonly accepted techniques using 22-mm square cover slips.

Relative abundance estimates were based on the method introduced by Hay for DSDP Leg 4 (1970). Letters on the range charts (Tables 111) denote nannofossil abundances and are keyed to the ${ }^{10} \log$ of the number of specimens of a particular taxon likely to be observed in any one field of view of the microscope at a magnification of $\times 1250$. These and the corresponding logs are designated as follows:
$\mathrm{H}=$ Highly abundant, +2 (more than 100 specimens per field of view)

$\mathrm{V}=$ Very abundant, +1 (more than 10 specimens per field of view)

$A=$ Abundant, $O$ ( 1 to 10 specimens per field of view)

$\mathrm{C}=$ Common,-1 ( 1 specimen per 2 to 10 fields of view $)$

$\mathrm{F}=\mathrm{Few},-2$ (1 specimen per 11 to 100 fields of view)

$\mathrm{R}=\mathrm{Rare},-3$ (1 specimen per 101 to 1000 fields of view)

Tabulations of reworked Cenozoic species and reworked Mesozoic species recorded on the range charts are also included employing the abundance criteria shown above. No attempt was made to subdivide the reworked Mesozoic taxa into a biozonational scheme.

The in situ/reworked ratio was calculated by dividing the relative abundance of indigenous taxa by the relative abundance of the reworked taxa. This ratio has theoretical limits from zero (all reworked taxa) to infinity (all indigenous taxa). One advantage of the in situ/reworked ratio is that it normalizes for differences in the density of the nannofossil solution dispersed on the cover slip. Although the relative abundances of the species will vary, the in situ/reworked ratio remains constant for both thick and thin nannofossil density dispersions. A control test made on 20 slides of varying densities processed from the same sample confirms this relationship. The total abundances observed in these slides ranged from less than 1 specimen per field of view to over 100 specimens per field of view. Calculations of the in situ/reworked ratio, however, consistently yielded values of approximately 100 .

In calculating the in situ/reworked ratio, the relative abundance estimates of very rare indigenous Pleistocene taxa were made on the average number of specimens likely to be observed in one traverse across a 22 -mm cover slip. For more abundant samples, relative abundances based on the number of specimens likely to be observed in any one field of view were converted to abundance per one traverse by multiplying by a factor of 150 (the approximate number of fields of view per traverse at a magnification of $\times 1250$ ). Although the precision of the ratios would be greatly improved by using data based on direct counts, the method outlined above is adequate to document the variations of the relative abundances of indigenous and reworked taxa observed during routine microscope examinations.

Selected samples near the datum level thought to represent deepest stratigraphic occurrence of dominant Emiliania huxleyi and the total depths of the holes were studied by scanning electron microscopy (SEM) to confirm the abundance and presence of E. huxleyi. Samples processed for SEM photography were concentrated and cleaned with a centrifuge settling technique.

The overall preservation of all samples examined is considered to be moderate. A majority of the specimens are slightly etched with the fine structures missing. No diagnostic morphological changes are visible under light microscopy.

\section{CALCAREOUS NANNOFOSSIL ZONATION}

The Gulf Coast and Caribbean-Antillean Zonation for the latest Pliocene through Recent of Boudreaux and Hay (1967) was used to make zonal age assignments. Modifications made to this zonation by Gartner and Emiliani (1976) were employed in the sites in which the datum marked by the lowest dominant occurrence of Emiliania huxleyi could be identified. Gartner and Emiliani subdivided the E. huxleyi Zone of Boudreaux and Hay into two zones: (1) the E. huxleyi Zone defined from the lowest occurrence of $E$. huxleyi to the lowest level of dominant $E$. huxleyi and (2) the E. huxleyi Acme Zone defined as the interval of dominant E. huxleyi (Fig. 2).

The identification of $E$. huxleyi specimens under the light microscope is provisional because of their small size and the difficulty in distinguishing between them and a similar species, Gephyrocapsa protohuxleyi. These factors, in conjunction with the rarity of indigenous Pleistocene species in most of the late Wisconsin glacial sediments, necessitates the use of the broader zonation of Boudreaux and Hay at most sites. 


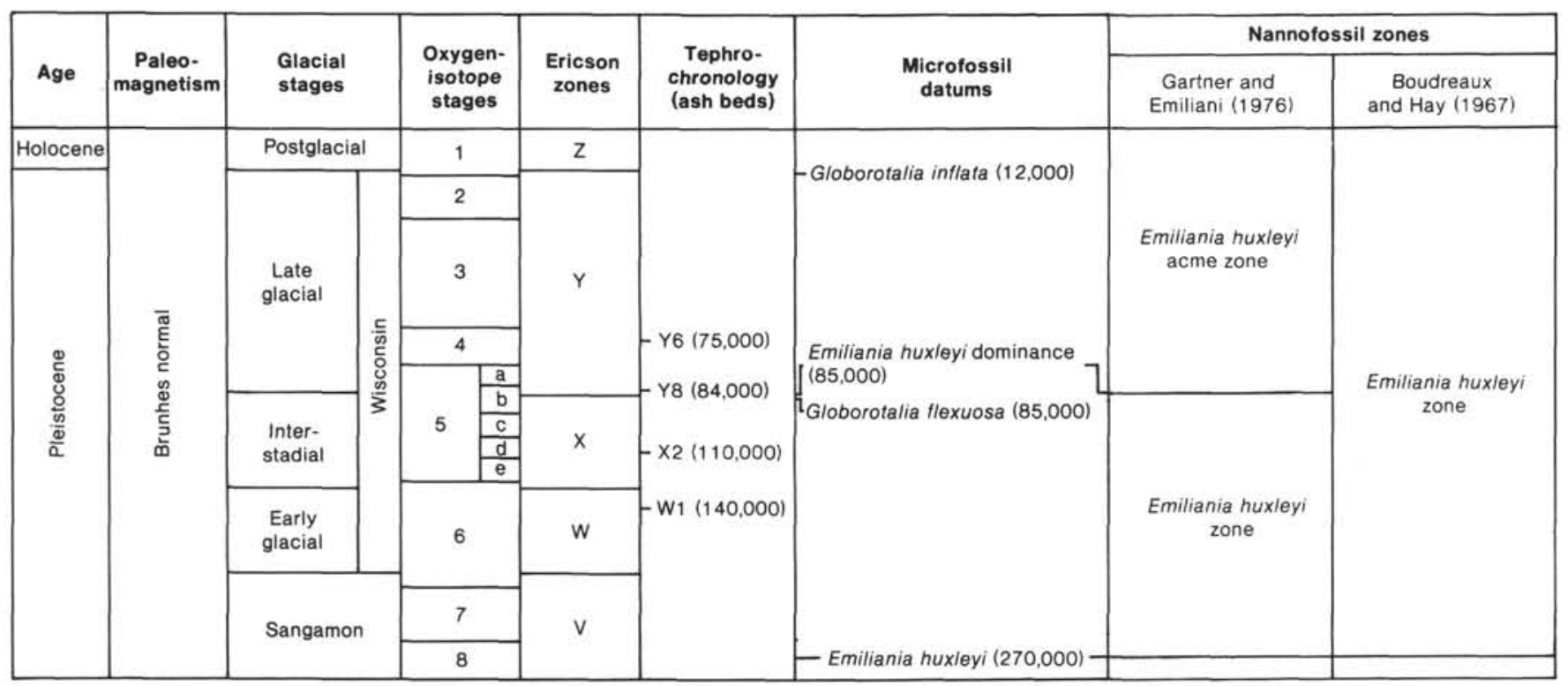

Figure 2. Late Quaternary zonation for the Gulf of Mexico. (From Emiliani, 1966, 1971; Boudreaux and Hay, 1967; Ericson and Wollin, 1968; Blow, 1969; Kennett and Huddleston, 1972; Rögl and Bolli, 1973; Gartner and Emiliani, 1976; Thierstein et al., 1977; Ledbetter, 1984; Williams, 1984.) The dates shown in tephrochronology and microfossil datums columns are in years ago.

In tropical and subtropical waters, the event marked by the reversal in dominance of $G$. caribbeanica and $E$. huxleyi has been associated with oxygen-isotope Substages $5 \mathrm{~b}-5 \mathrm{a}$ at approximately 85,000 yr. ago by Thierstein et al. (1977). Williams (1984) also correlated the top of the Ericson Zone X, marked by the last occurrence of the planktonic foraminifer Globorotalia flexuosa, with isotope Substage $5 \mathrm{~b}$. The Y8 ash of Kennett and Huddleston (1972) occurs just above this event and has been dated at 84,000 yr. ago in association with Substage 5 b in Gulf of Mexico sediments (Ledbetter, 1984). These correlations are shown in Figure 2.

\section{SITE SUMMARIES}

Leg 96 investigated the Mississippi Fan and two intraslope basinal depressions between diapirically intruded salt masses. The Mississippi Fan is a broad, thick arcuate deposit of displaced Pleistocene sediments on the abyssal floor of the Gulf of Mexico. The youngest fan lobe has a large sinuous central channel that decreases in width and depth in the lower fan regions (introductory chapter, this volume). Although the site discussions are grouped by depositional province, each site is considered separately. Distribution charts are presented for all sites (Tables 1-11).

\section{Middle Fan Sites}

The middle fan region of the present Mississippi Fan is generally located between water depths of 2100 and $3100 \mathrm{~m}$. The four sites cored in this area (Sites 617,620 , 621 , and 622 ) provide a transit from the western levee, across the channel, and onto an overbank region (Fig. 1). In this region, located approximately $300 \mathrm{~km}$ from the present Mississippi River Delta, the channel is 1.5 to $2.5 \mathrm{~km}$ wide and bounded by prominent ridges or levees (Middle Fan Introduction and Summary, this volume).

\section{Site 621}

Site $621\left(26^{\circ} 43.86^{\prime} \mathrm{N}, 88^{\circ} 29.76^{\prime} \mathrm{W}\right.$; water depth, $2485 \mathrm{~m}$ ) was hydraulic piston cored to a sub-bottom depth of $214.8 \mathrm{~m}$ to investigate an outer convex meander bend (thalweg) of the present channel. The recovered channelfill sediments show a fining-upward trend from a basal gravel through sand, silt-mud laminates, into thick-bedded muds. The upper $50 \mathrm{~m}$ consist primarily of homogeneous muds (Site 621 chapter, this volume).

The $0.25 \mathrm{~m}$ of a marly foraminiferal ooze recovered at the top of Core 621-1 was the only interval containing abundant, moderately well-preserved Quaternary nannofossils (Table 1). Most common of these are Emiliania huxleyi, Crenalithus doronicoides, and Gephyrocapsa spp. (small). Reworked Cretaceous nannofossils are very rare.

In the sediments below this upper Holocene ooze, the nannofossil assemblages are dominated by common reworked Cretaceous species. Pleistocene taxa, when present, are rare. SEM identification of small coccoliths in Core $621-33$ as $E$. huxleyi dictates that all cores recovered at this site be placed in the E. huxleyi Zone.

The values of the in situ/reworked ratio generally increase uphole from low values of 0.01 to a high of 100 in the Holocene veneer (Fig. 3). This suggests that sedimentation rates at this site were decreasing throughout the late Wisconsin glacial stage.

\section{Site 622}

Site $622\left(26^{\circ} 41.41^{\prime} \mathrm{N}, 88^{\circ} 28.82^{\prime} \mathrm{W}\right.$; water depth, $2495 \mathrm{~m}$ ) was hydraulic piston cored in the inner concave meander bend ("point bar") of the present channel. The total depth of penetration was $208.0 \mathrm{~m}$ sub-bottom. The lithologic sequence recovered, with the exception of a thicker, sandy basal section, is similar to that at Site 621 (Site 622 chapter, this volume). 
Table 1. Distribution of calcareous nannofossils, Site 621 .

\begin{tabular}{|c|c|c|c|c|c|c|c|c|c|c|c|c|c|c|c|c|c|c|c|c|c|c|c|c|c|c|c|}
\hline $\begin{array}{c}\text { Core- } \\
\text { Section } \\
\text { (interval } \\
\text { in } \mathrm{cm} \text { ) }\end{array}$ & $\begin{array}{c}\text { Sub- } \\
\text { bottom } \\
\text { depth } \\
\text { (m) }\end{array}$ & 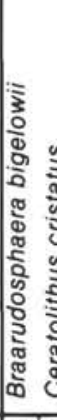 & 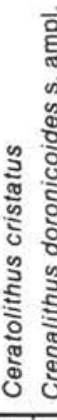 & & & 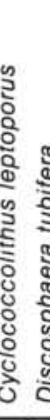 & 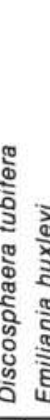 & 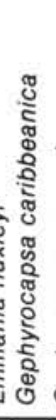 & & & & & & & 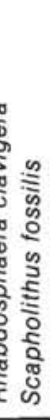 & & 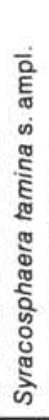 & & & 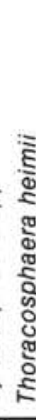 & & & & 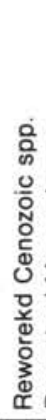 & 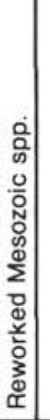 & 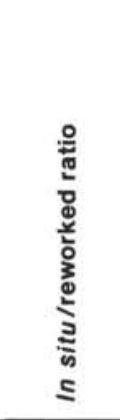 & $\begin{array}{l}\text { Zone or } \\
\text { subzone }\end{array}$ \\
\hline $1-1.21-22$ & 22 & $\begin{array}{lll}R & F \\
\end{array}$ & \begin{tabular}{l|l}
$R$ & $F$ \\
\end{tabular} & $R$ & \begin{tabular}{|l|l|l|}
$R$ \\
\end{tabular} & $\begin{array}{ll}R & R \\
\end{array}$ & $\begin{array}{ll}R & C \\
\end{array}$ & $R$ & \begin{tabular}{|l|l|}
$R$ & $F$ \\
\end{tabular} & $\bar{F} \mathrm{R}$ & $R$ & $R$ & & \begin{tabular}{l|l}
$R$ & $R$ \\
\end{tabular} & $R$ & & & F & $R$ & $R$ & & $\mathrm{R}$ & $R$ & & $\mathrm{R}$ & 100 & \multirow{48}{*}{$\begin{array}{c}\text { Emiliania } \\
\text { huxleyi }\end{array}$} \\
\hline $1-1,55-56$ & .56 & & $\mathrm{~F}$ & & & & $F$ & $R$ & $R$ & $\begin{array}{l}\mathrm{R} \\
\end{array}$ & & & & & & & & & & & & & & & $F$ & .1 & \\
\hline $1-2.45-46$ & 1. 96 & & F & & & & $\mathrm{F}$ & $R$ & \begin{tabular}{l|l}
$R$ & $F$ \\
\end{tabular} & \begin{tabular}{l|l}
$R$ & $R$ \\
\end{tabular} & $\mathrm{R}$ & & & & $R$ & & $R$ & F & $R$ & & $R$ & F & & & $F$ & 2 & \\
\hline $1-3,17-18$ & 3. 18 & & F & & & $\mathrm{R}$ & F & $R$ & $\begin{array}{ll}R \\
\end{array}$ & R & $\mathrm{R}$ & & & $R$ & $R$ & & & & & $\mathrm{R}$ & & $F$ & & & $F$ & 1. & \\
\hline $2-1, \quad 38-39$ & 3. 88 & & $F$ & & & $R$ & $F$ & $R$ & & $R$ & & & & & & & & $F$ & & & & & & & $F$ & .25 & \\
\hline $2-3 . \quad 38-39$ & 6. 89 & & $\mathrm{R}$ & & $R$ & \begin{tabular}{l|l}
$R$ \\
\end{tabular} & $\mathrm{~F}$ & $R$ & & $R$ & & & & & 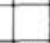 & & & & & & & & & & $F$ & 1 & \\
\hline $2-5, \quad 38-39$ & 9.89 & & $\mathrm{~F}$ & & $R$ & F & \begin{tabular}{l|l}
$R$ & $F$ \\
\end{tabular} & $R$ & $R F$ & $R$ & & & & & $R$ & & & & & & & & $R$ & & $F$ & 5. & \\
\hline $2-6, \quad 38-39$ & 11.39 & & $\mathrm{R}$ & & $R$ & $R$ & $F$ & $R$ & R $R$ & $R$ & & & & & & & $R$ & & $R$ & & & & & & $F$ & 4. & \\
\hline $3-1,16-17$ & 13.27 & & $\mathrm{F}$ & $R$ & $R$ & & $\mathrm{~F}$ & $R$ & $P_{F}$ & $R$ & & $R$ & & & & & & & & & & & & & $F$ & .25 & \\
\hline $3-3,40-41$ & 16.51 & $R$ & $\mathrm{R}$ & & & & $\mathrm{F}$ & $R$ & $R$ & & & & & & $t$ & & & & & & & & & & $F$ & 1. & \\
\hline $3-5,40-41$ & 19.51 & $\mathrm{R}$ & $R$ & & . & & $\mathrm{F}$ & $R$ & $R$ & 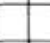 & & & & & & & & & & & & & & & $F$ & 2. & \\
\hline $3-6.40-41$ & 21.01 & & $\mathrm{R}$ & & & & $\mathrm{F}$ & & $R F$ & $R$ & & & & & & & & & & & & & & & C & 1. & \\
\hline $4-1,40-41$ & 23. 11 & & $\mathrm{R}$ & & 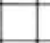 & & $\mathrm{F}$ & & $\begin{array}{lll} & F \\
\end{array}$ & \begin{tabular}{l|l}
$R$ & $R$ \\
\end{tabular} & $\mathrm{R}$ & & & & & & & & & & & & & & $F$ & 1 & \\
\hline $4-3,40-41$ & 26. 11 & & $\mathrm{R}$ & & & & $\mathrm{F}$ & & \begin{tabular}{l|l}
$R$ & $F$ \\
\end{tabular} & \begin{tabular}{l|l}
$R$ & $F$ \\
\end{tabular} & R & & & & & & & & & & & & & & $F$ & .2 & \\
\hline $4-5, \quad 40-41$ & 29. 11 & & & & & & $\mathrm{~F}$ & $R$ & & $R$ & & & & & & & & $\mathrm{~F}$ & & & & & & & $F$ & 1.5 & \\
\hline $5-1,40-41$ & 32.71 & & $\mathrm{R}$ & & & & $\mathrm{F}$ & & F & $R$ & & & & & 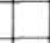 & $R$ & $R$ & F & & $R$ & & & . & & $F$ & 1. & \\
\hline $5-3,40-41$ & 35.71 & & $\mathrm{R}$ & & & & $\mathrm{F}$ & $R$ & $R$ & & & & & & 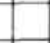 & & & & & & & & $R$ & & C & 2 & \\
\hline $5-5,40-41$ & 38.71 & & & & & & $\mathrm{~F}$ & $R$ & & $\mathrm{R}$ & & & & & 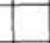 & & & & & & & & & & $F$ & 5 & \\
\hline $6-1,40-43$ & 42.31 & & $\mathrm{R}$ & & & & $\mathrm{F}$ & $R$ & F & $R$ & & & & & $R$ & & & 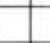 & & $R$ & & & . & & C & 2 & \\
\hline $6-3,40-41$ & 45.31 & & $\mathrm{~F}$ & & & & $\mathrm{~F}$ & $R$ & $R$ & & & & & & $R$ & & & & & & & & $R$ & $R$ & C & .2 & \\
\hline $7-1,40-41$ & 46.61 & & $R$ & & & & c & $R$ & $R$ & & $\mathrm{R}$ & & & $R$ & $R$ & & & $\mathrm{~F}$ & & & & $R$ & $R$ & $R$ & C & 5 & \\
\hline $7-3,40-41$ & 49.61 & & $\mathrm{~F}$ & & & & $\mathrm{~F}$ & & & $R$ & & & & & & & & & & & & & & & C & 1 & \\
\hline $7-5, \quad 40-41$ & 52.61 & & & & & & $\mathrm{~F}$ & & & $\mathrm{R}$ & & & & & $R$ & & & & & & & & & & c & 2 & \\
\hline $8-1,40-41$ & 56.11 & & \begin{tabular}{l|l}
$R$ & $R$ \\
\end{tabular} & & & & $\mathrm{~F}$ & $R$ & & $R$ & & & & & & & & & & & & & & & C & 1 & \\
\hline $8-3,40-41$ & 59.11 & & $\mathrm{R}$ & & & & F & & $R F$ & $\mathrm{R}$ & & & & & & & & & & & & $\mathrm{R}$ & & & C & 2 & \\
\hline $9-1,40-41$ & 60.61 & & $R$ & & & & $\mathrm{~F}$ & & & $R$ & & & & & & & & & & & & & & & $F$ & -1 & \\
\hline $9-3.40-41$ & 63.61 & & $\mathrm{R}$ & & & $R$ & $F$ & & \begin{tabular}{|l|l|l}
$R$ & $F$ \\
\end{tabular} & $R$ & & & & $R$ & $R$ & & & & & & & & $\mathrm{R}$ & & $F$ & 5 & \\
\hline $10-1,40-41$ & 65.61 & & $\mathrm{R}$ & & & & $\mathrm{F}$ & & & $R$ & & & & & & & & $F$ & & & & & $R$ & & $F$ & .25 & \\
\hline $10-3, \quad 40-41$ & 68.61 & & $R$ & & & & $\mathrm{~F}$ & $R$ & & $R$ & & & & & & & & & & & & & & & $\mathrm{~F}$ & .1 & \\
\hline $11-1.40-41$ & 70.81 & & $\mathrm{R}$ & & $R$ & & $\mathrm{R}$ & $R$ & & $\mathrm{R}$ & $\mathrm{R}$ & & & & & & & & & & & & & & $F$ & 2 & \\
\hline $11-3,40-41$ & 73. 81 & & $R$ & & & & $\mathrm{~F}$ & & & $\mathrm{R}$ & & & & & & & & & & & & & & & $F$ & 15 & \\
\hline $11-5,40-41$ & 76.81 & & $\mathrm{~F}$ & & $R$ & & \begin{tabular}{l|l}
$R$ & $C$ \\
\end{tabular} & $R$ & & $R$ & $R$ & & $R$ & & & & & & & $R$ & & & & & C & 2 & \\
\hline $12-1.40-41$ & 79.21 & & $\mathrm{~F}$ & & & & $\mathrm{R}$ & & & $\mathrm{R}$ & & & & & & & & & & & & & & & $F$ & .1 & \\
\hline $12-2, \quad 40-41$ & 80.71 & & $R$ & & & & $\mathrm{R}$ & $R$ & & $\mathrm{~F}$ & \begin{tabular}{l|l}
$R$ & $R$ \\
\end{tabular} & & & & & & & $R$ & & & & & & & C & .05 & \\
\hline $13-1,40-41$ & 81.81 & & $\mathrm{R}$ & & & & $R$ & $R$ & & \begin{tabular}{l|l}
$\mathrm{R}$ & $\mathrm{R}$ \\
\end{tabular} & R & & & & & & & & & & & & & & $F$ & $\therefore 1$ & \\
\hline $13-3,40-41$ & 84. 81 & & $R$ & & & & $R$ & $R$ & & $\begin{array}{ll}\mathrm{R} \\
\end{array}$ & & & & & & & & & & & & & & & $F$ & 1 & \\
\hline $14-1,40-41$ & 87. 11 & & & & & & $\mathrm{~F}$ & $R$ & $R$ & & & & & & & & & & & & & & & & $F$ & .1 & \\
\hline $14-3,40-41$ & 90.11 & & $R$ & & & & $\mathrm{~F}$ & $R$ & & $\mathrm{R}$ & & & & & & & & & & & & & & & c & .05 & \\
\hline $14-5, \quad 40-41$ & 93.11 & & & & & & F & $R$ & $R$ & & & & & & & & & & & & & & & & $c$ & .05 & \\
\hline $15-1,40-41$ & 94.61 & $R$ & $\mathrm{R}$ & & & & $R$ & $R$ & & & & & & & R & & & & & & & & & & C & .05 & \\
\hline $15-3,40-41$ & 97.61 & & $\mathrm{R}$ & & & & $\mathrm{R}$ & & & & & & & & & & & F & & & & & $\mathrm{R}$ & & C & .05 & \\
\hline $16-1.40-41$ & 100.11 & & $\mathrm{R}$ & & & & $\mathrm{F}$ & $R$ & & $\mathrm{R}$ & & & & & & & & F & & & & & & & C & .05 & \\
\hline $16-3,40-41$ & 103.11 & & $\mathrm{R}$ & & $R$ & & $\mathrm{R}$ & $R$ & $R$ & & & & & & & & & & & & & & & & $F$ & .05 & \\
\hline $17-1.40-41$ & 105.11 & & & & $R$ & & $\mathrm{R}$ & $R$ & & $\mathrm{R}$ & & & & & & & & & R & & & & & & C & .05 & \\
\hline $17-2, \quad 40-41$ & 106.61 & & $\mathrm{R}$ & & & & $\mathrm{F}$ & $R$ & & $\mathrm{R}$ & & & & & & & & & & & & & & & C & .1 & \\
\hline $18-1 . \quad 40-41$ & 114.61 & & $R$ & & & & $\mathrm{R}$ & & & & & & & & & & & $\mathrm{F}$ & & & & & & & $R$ & .1 & \\
\hline $19-1,40-41$ & 124.11 & & \begin{tabular}{l|l}
$R$ & $F$ \\
\end{tabular} & & & & $R$ & $R$ & & $R$ & & & & & $R$ & $R$ & & & & $R$ & $R$ & & & & $F$ & 15 & \\
\hline $19-3 . \quad 40-41$ & 127.11 & & \begin{tabular}{l|l}
$R$ & $R$ \\
\end{tabular} & & $R$ & & $F$ & $R$ & \begin{tabular}{|l|l|l|}
$R$ & \\
\end{tabular} & R & 8.7 & $R$ & $R$ & \begin{tabular}{l|l}
$R$ & $R$ \\
\end{tabular} & & & & $\mathrm{R}$ & $\mathrm{R}$ & & & & & & $F$ & .2 & \\
\hline
\end{tabular}


Table 1 (continued).

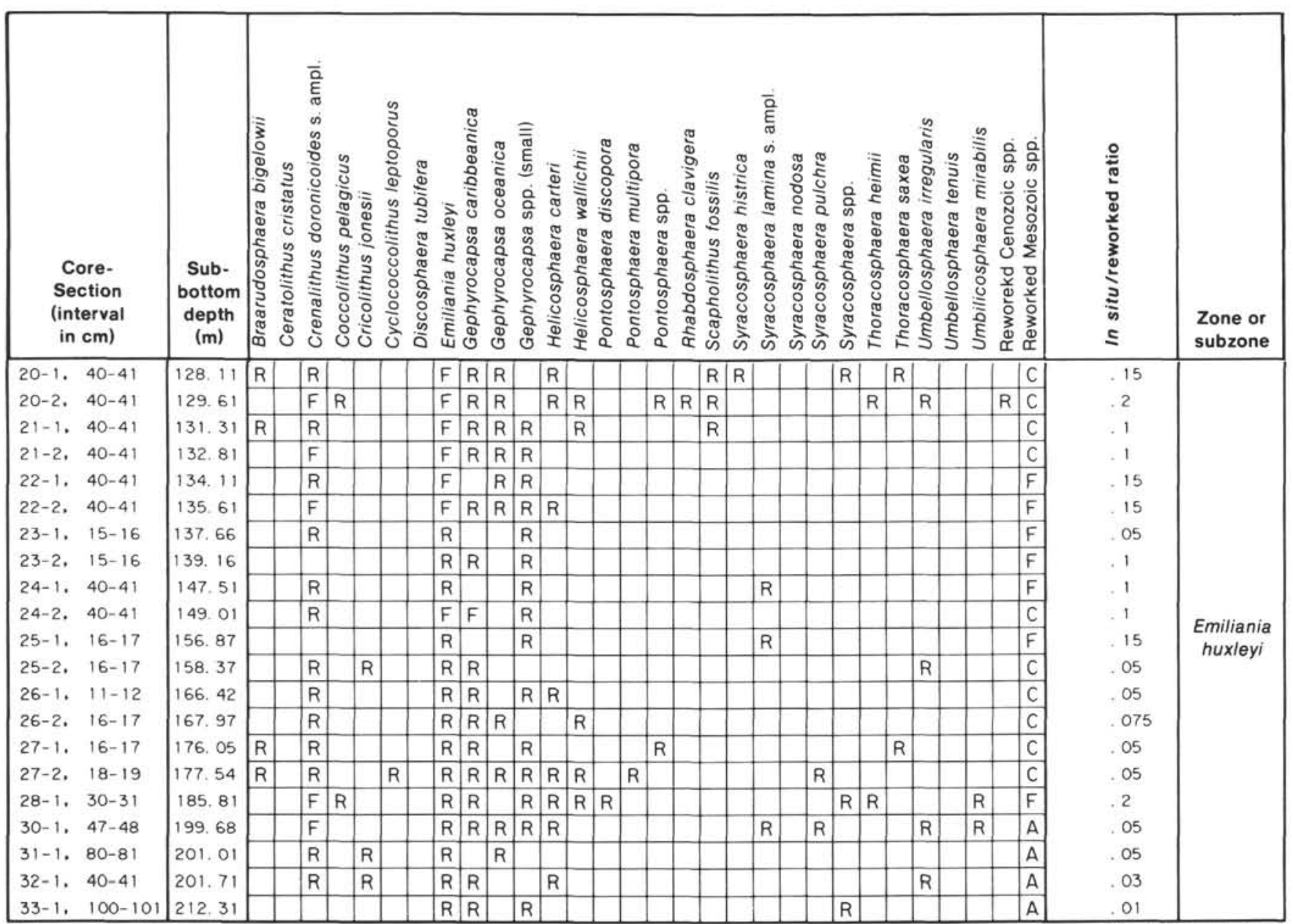

Note: For abundance, $A=$ abundant, $1-10$ specimens/field; $C=$ common, 1 specimen $/ 2-10$ fields; $F=$ few, 1 specimen $/ 11-100$ fields; $R=$ rare, 1 specimen $/ 101-1000$ fields (when viewed at a magnification of $\times 1250$ ).

Nannofossils common in the marly foraminiferal ooze in the top few centimeters of Section 622-1-1 include most taxa that are typical of Holocene assemblages in the Gulf of Mexico (Pierce and Hart, 1979) (Table 2). Below this ooze, the floral assemblage is dominated by reworked Cretaceous species. Gephyrocapsa oceanica, G. caribbeanica, and Emiliania huxleyi are the most frequently occurring Pleistocene nannofossils.

The in situ/reworked ratio curve for this site is very similar to Site 621 (Fig. 3). Slight increases in the ratio to values of 0.3 to 0.4 in Cores 622-12 through 16 (105$135 \mathrm{~m}$ sub-bottom) suggest a minor temporary decrease in the sedimentation rate for this interval. The mud and clay sediments above this zone probably represent deposition during the waning phase of the late Wisconsin glacial.

\section{Site 617}

Site $617\left(26^{\circ} 41.93^{\prime} \mathrm{N}, 88^{\circ} 31.67^{\prime} \mathrm{W}\right.$; water depth $2478 \mathrm{~m}$ ) was hydraulic piston cored in a ridge and swale topography immediately adjacent to the channel and penetrated $191.2 \mathrm{~m}$ of overbank deposits. The vertical sequence of muds with silt laminations initially increases in grain size, then exhibits a fining-upward trend into homogeneous mud (Site 617 chapter, this volume).

The surficial foraminiferal ooze was not recovered in Hole 617. Hole 617A, however, did contain a well-developed ooze, containing abundant, well-preserved calcareous nannofossils (Table 3). Reworked Cretaceous nannofossils are the major floral constituent in the remainder of Holes 617 and 617A.

Values of the in situ/reworked ratio remain low throughout the samples examined, suggesting a rapid sedimentation rate for all the fine-grained sediments deposited at this site. Calculations of the sedimentation rate based on seismic projections to seismic Horizon " 30 " (introductory chapter, this volume) indicate a rate in excess of $10 \mathrm{~m} / 1000 \mathrm{yr}$. for the late Wisconsin glacial period. Only one very slight increase in the abundance of Pleistocene nannofossils could be identified. This was found in the muddy interval sampled by Core 617-15 (132.5-135.5 m sub-bottom).

\section{Site 620}

Site $620\left(26^{\circ} 50.12^{\prime} \mathrm{N}, 88^{\circ} 22.25^{\prime} \mathrm{W}\right.$; water depth, $2612 \mathrm{~m}$ ), located in overbank deposits about $18 \mathrm{~km}$ from 


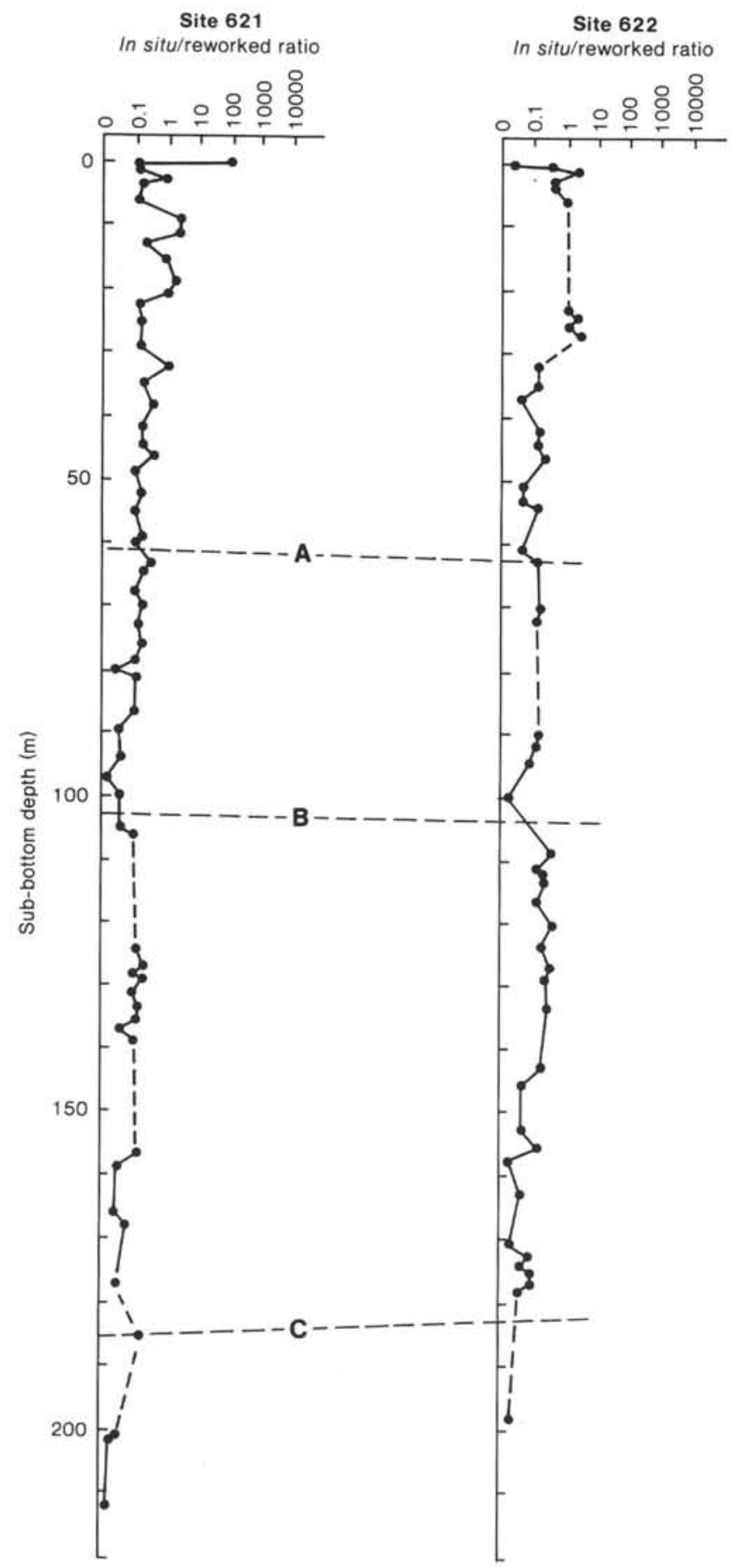

Figure 3. Correlation of the in situ/reworked ratio curves for Sites 621 and 622 . Lines A, B, and C indicate probable correlations between sites.

the channel-levee complex, was rotary cored with relatively poor recovery to a depth of $422.7 \mathrm{~m}$. These overbank deposits consist primarily of fine grained clay and silty clay arranged in alternating coarsening-upward sequences (Site 620 chapter, this volume). The overall clay percentage at this site is estimated at approximately $73 \%$.

All cores recovered from this site are interpreted to be in the Emiliania huxleyi Zone (Table 4). Well-preserved Quaternary nannofossils are abundant only in the thin
Holocene marly calcareous ooze. Below this ooze, the nannofossil assemblages are dominated by reworked Cretaceous species. Pleistocene nannofossils are rare, resulting in low in situ/reworked ratios.

Slight increases in the abundance of indigenous Pleistocene taxa are present. The most significant increase occurs near the bottom of the hole, between Cores 620-40 and $620-45$ (365-410 $\mathrm{m}$ sub-bottom). This increase coincides with the projection of seismic Reflector " 20 " at a sub-bottom depth of $371 \mathrm{~m}$ (introductory chapter, this volume). This interval, in which the in situ/reworked ratios may reach 10 , could represent a reduction of the sedimentation rate in response to a warmer pulse during oxygen-isotope Stage 3.

\section{Lower Fan Sites}

On the lower fan, the wide single channel-levee complex narrows to less than $500 \mathrm{~m}$ and changes to a less sinuous and channel-switching mode. Two sites (623 and $624)$ were drilled on the margins of this lower fan channel and two sites (615 and 614) were positioned near the distal end of the channel terminations (introductory chapter, this volume; Fig. 1).

\section{Site 623}

Site $623\left(25^{\circ} 46.09^{\prime} \mathrm{N}, 86^{\circ} 13.84^{\prime} \mathrm{W}\right.$; water depth, $3188 \mathrm{~m}$ ) was hydraulic piston cored to a total penetration of $202.2 \mathrm{~m}$ to evaluate the switching nature of the channel in the lower fan region. The recovered sediments consist of both fining-upward channel fill sequences and coarsening-upward overbank intervals consisting of thin sands, silts, and muds (Site 623 chapter, this volume).

The warm-water Holocene planktonic ooze unit is absent. Core 623-1 probably did not sample the sediment/ water interface. Pleistocene calcareous nannofossils are generally few in number (Table 5). The floral assemblages are dominated by moderately preserved reworked Cretaceous nannofossils. Increases in the abundance of indigenous Pleistocene taxa occur sporadically. One slight increase is noted in the muddy interval of Cores 621-13 through 621-14 (112-125 m sub-bottom). The in situ/reworked ratio values are generally low throughout all recovered sediments.

\section{Site 624}

Site $624\left(25^{\circ} 45.24^{\prime} \mathrm{N}, 86^{\circ} 16.63^{\prime} \mathrm{W}\right.$; water depth, $3198 \mathrm{~m}$ ), located $4.8 \mathrm{~km}$ from the channel, was cored with the advanced piston corer/extended core barrel (APC/ $\mathrm{XCB}$ ) (Explanatory Notes, this volume) to a depth of $199.9 \mathrm{~m}$ (Hole 624A). All cores recovered at this hole are assigned to the Emiliania huxleyi Zone (Table 6). Except for the presence of a thin $(6 \mathrm{~cm})$ Holocene marly foraminiferal ooze, the nature of the sediment recovered is very similar to Site 623 (Site 624 chapter, this volume). The marly ooze in the top of Core 624-1 contains a moderately well-preserved nannofossil assemblage dominated by $E$. huxleyi; in situ/reworked ratios can reach 6,000 (Table 6).

As at Site 623, the interval below the Holocene ooze is dominated by reworked Cretaceous species. The generally low in situ/reworked ratios are a function of the 
Table 2. Distribution of calcareous nannofossils, Site 622.

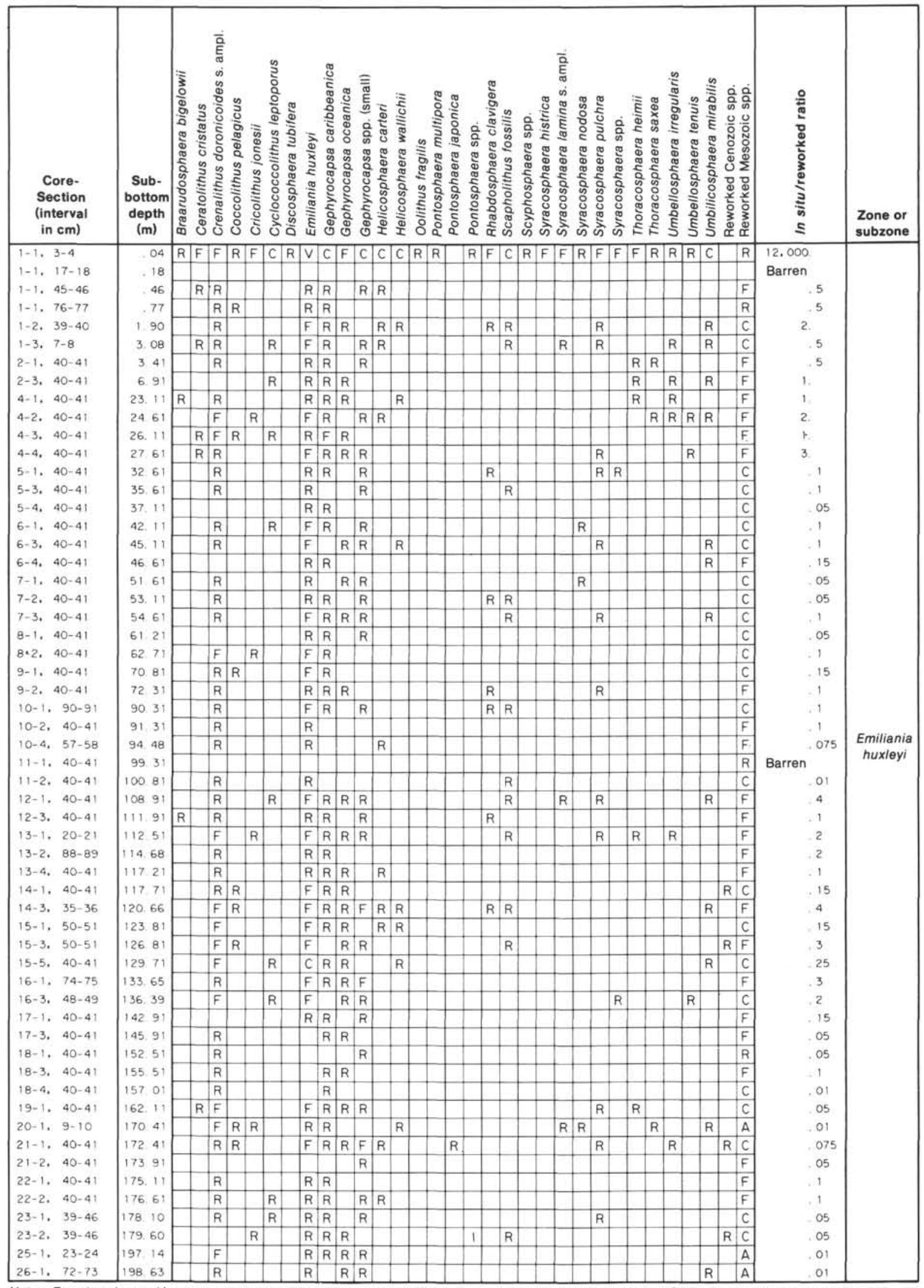

Note: For abundance, $V=$ very abundant, 10-100 specimens/field; $A=$ abundant, $1-10$ specimens $/$ field; $C=$ common, 1 specimen $/ 2-10$ fields; $F=$ few. 1 specimen $/ 11-100$ fields; $R=$ rare, 1 specimen $/ 101-1000$ fields (when viewed at a magnification of $\times 1250$ ). 


\section{R. E. CONSTANS, M. E. PARKER}

Table 3. Distribution of calcareous nannofossils, Site 617.

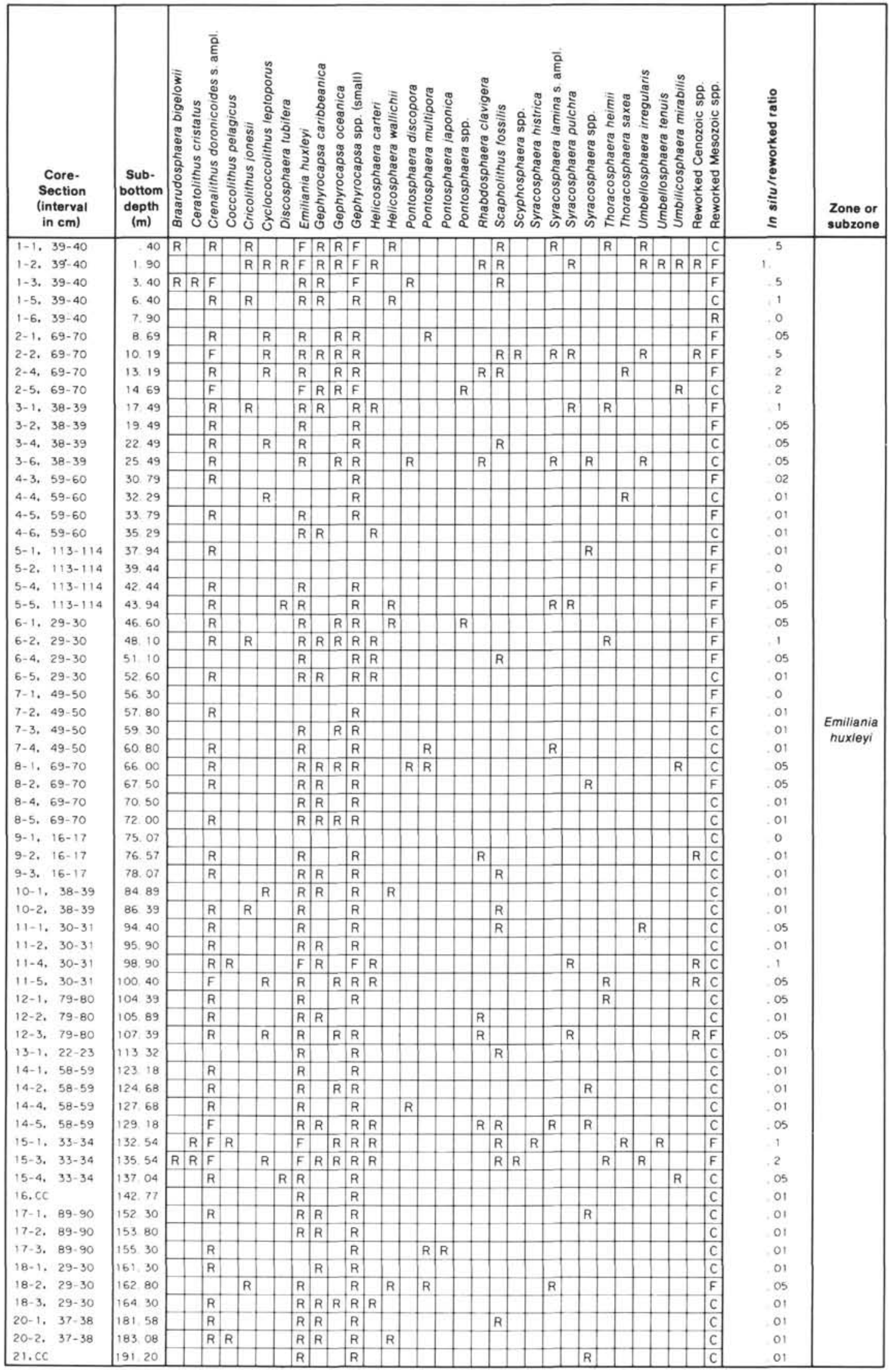

Note: For abundance, $\mathrm{A}=$ abundant, $1-10$ specimens/field: $\mathrm{C}=$ common, 1 specimen $/ 2-10$ fields; $\mathrm{F}=1$ few, 1 specimen $/ 11-100$ fields, $\mathrm{R}=$ rare, 1 specimen $/ 101-1000$ fields (when viewed at a magnification of $\times 1250$ ). 
Table 4. Distribution of calcareous nannofossils, Site 620 .

\begin{tabular}{|c|c|c|c|c|c|c|c|c|c|c|c|c|c|c|c|c|c|c|c|c|c|c|c|}
\hline $\begin{array}{l}\text { Core- } \\
\text { Section } \\
\text { (interval } \\
\text { in } \mathrm{cm} \text { ) }\end{array}$ & $\begin{array}{l}\text { Sub- } \\
\text { bottom } \\
\text { depth } \\
(\mathrm{m})\end{array}$ & 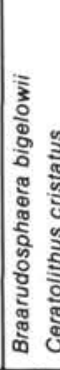 & 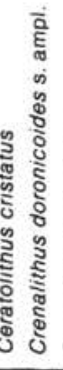 & 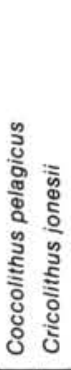 & 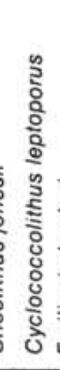 & & 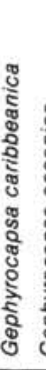 & 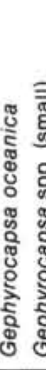 & 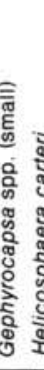 & 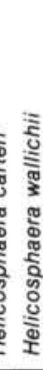 & & 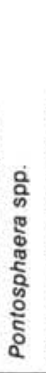 & 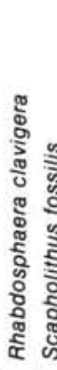 & 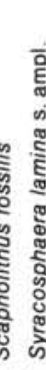 & 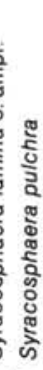 & 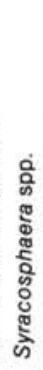 & 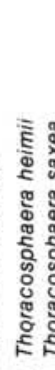 & 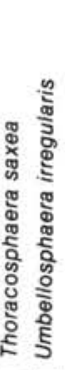 & 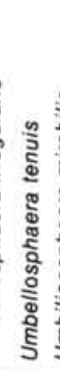 & 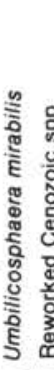 & 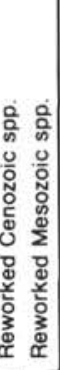 & 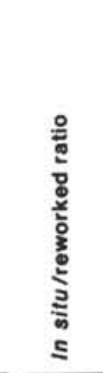 & $\begin{array}{l}\text { Zone or } \\
\text { subzone }\end{array}$ \\
\hline $1-1 \cdot 21-22$ & .22 & R & \begin{tabular}{l|l|}
$R$ & $F$ \\
\end{tabular} & $F$ & $F$ & \begin{tabular}{|l|l}
$C$ & $P$ \\
\end{tabular} & \begin{tabular}{|l|l|}
$F$ \\
\end{tabular} & \begin{tabular}{l|l}
$F$ & $F$ \\
\end{tabular} & \begin{tabular}{l|l}
$R$ & $F$ \\
\end{tabular} & \begin{tabular}{l|l}
$F$ \\
\end{tabular} & $R$ & & \begin{tabular}{|l|l}
$R$ & $F$ \\
\end{tabular} & \begin{tabular}{l|l}
$F$ & $R$ \\
\end{tabular} & & & & $R$ & & $F$ & $F$ & 8. & \multirow{51}{*}{$\begin{array}{l}\text { Emiliania } \\
\text { huxieyi }\end{array}$} \\
\hline $1-2,43-44$ & 1. 94 & & $F$ & $R$ & & $\mathrm{FP}$ & $\mathrm{F}$ & & & & & & \begin{tabular}{l|l} 
\\
\end{tabular} & & $R$ & & & & & & C & 1 & \\
\hline $2-1.39-40$ & 3. 40 & & $R$ & & & \begin{tabular}{|l|l|l}
$F$ & $P$ \\
\end{tabular} & $\mathrm{~F}$ & & $F$ & $\mathrm{R}$ & & & & & & & & & & & A & .05 & \\
\hline $2-3,39-40$ & 6. 40 & & & & & $\mathrm{R}$ & & \begin{tabular}{l|l}
$\mathrm{R}$ & $\mathrm{F}$ \\
\end{tabular} & $R$ & & & & & & 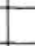 & & & & & & A & 01 & \\
\hline $2-5, \quad 39-40$ & 9. 40 & & $F$ & & & $\mathrm{~F} / \mathrm{F}$ & $\mathrm{F}$ & & $F$ & R & & & & & $R$ & & & & & & A & .05 & \\
\hline $3-1,40-41$ & 13.01 & & $R$ & & & $R$ & & $R$ & & & & & & & & & & 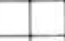 & & & A & 01 & \\
\hline 3-3. $40-41$ & 16. 01 & & $R$ & $R$ & & & $\mathrm{R}$ & & & & & & & & & & & $R$ & & & C & 01 & \\
\hline $4-2.40-41$ & 24. 11 & $\mathrm{R}$ & $R$ & & & $\mathrm{RF}$ & $\mathrm{R}$ & $R$ & $F$ & $R$ & & & & & & & & $R$ & $R$ & & C & .05 & \\
\hline $4-3 \cdot 40-41$ & 25.61 & & & & & $R$ & & $R F$ & \begin{tabular}{l|l}
$R$ \\
\end{tabular} & & & & & & & & & & $R$ & & C & .01 & \\
\hline $5-1.110-111$ & 32.91 & & $\mathrm{R}$ & & & $\mathrm{R}$ & & & & & & & 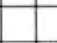 & & & & & & & & A & .025 & \\
\hline $5-3 \cdot 40-41$ & 33.71 & & $\mathrm{R}$ & & & f & $R$ & & & & & & F & & & & & & & & A & .025 & \\
\hline $6-1.40-41$ & 41.81 & & E & & & $\mathrm{R} / \mathrm{H}$ & $\mathrm{R}$ & & & & & & & & & & & & & & A & 01 & \\
\hline $6-3,40-41$ & 44. 81 & & $R$ & & & $R$ & & $R F$ & $R$ & & & & & & & & & & & & C & 02 & \\
\hline $7-1.40-41$ & 51,41 & & $R$ & $R$ & & $\mathrm{~F} / \mathrm{F}$ & $R$ & $R$ & & & & & & & & & & & & $R$ & C & .03 & \\
\hline $7-3,40-41$ & 54.41 & & $\mathrm{~F}$ & & & $\mathrm{~F} F$ & $R$ & \begin{tabular}{l|l}
$R$ & $F$ \\
\end{tabular} & $R$ & & & & & & & & & & & & C & .05 & \\
\hline $8-1.40-41$ & 61.01 & & & & & $\mathrm{R}$ & $R$ & & & & & & & & & & & & & & A & .01 & \\
\hline $9-2,40-41$ & 72.11 & & $R$ & & & $R$ & & $\rightarrow$ & & & & & & & & & & & & & A & .01 & \\
\hline $9-5,40-41$ & 76.61 & & $\mathrm{~F}$ & & & $R$ & $R$ & $R$ & & & & & & & & & & & & & C & .05 & \\
\hline $9-6,40-41$ & 78. 11 & & + & & & $R$ & \begin{tabular}{l|l}
$R$ \\
\end{tabular} & & $\mathrm{~F}$ & $\mathrm{R}$ & & & & & & & & & & & A & .025 & \\
\hline $10-1,40-41$ & 80.21 & & $R$ & & & $R$ & \begin{tabular}{|l|l}
$\mathrm{R}$ \\
\end{tabular} & & $\mathrm{R}$ & & & & & & & & & & & & C & 025 & \\
\hline $10-3, \quad 40-41$ & 83.21 & & $R$ & & & $R$ & & \begin{tabular}{l|l}
$\mathrm{R}$ & $\mathrm{R}$ \\
\end{tabular} & & & & & & & & & & & & & A & 025 & \\
\hline $11-1,40-41$ & 89.81 & & $R$ & & & $R$ & $R$ & $\mathrm{R}$ & ti & \begin{tabular}{l|l} 
\\
\end{tabular} & & & & & & & & & & $R$ & A & .025 & \\
\hline $12-1,110-111$ & 99.71 & & $\mathrm{~F}$ & & & $R$ & $\mathrm{~F}$ & & $\mathrm{R}$ & $R$ & & $R$ & & R & & $R$ & & & & $R$ & A & .01 & \\
\hline $12-4, \quad 40-41$ & 10351 & & $\mathrm{~F}$ & $R$ & $R$ & $F$ & $\mathrm{R}$ & \begin{tabular}{l|l}
$R$ & $F$ \\
\end{tabular} & $R$ & & & & & & $R$ & & & & & & C & 1 & \\
\hline $12-6.40-41$ & 106.51 & & $R$ & & & $F$ & $\mathrm{R}$ & & $R$ & & & & & & & & & & & & A & 05 & \\
\hline $13-1, \quad 40-41$ & 108.21 & & $R$ & & & $R$ & & & & & & & & & T & & & & & & A & 01 & \\
\hline $13-3, \quad 40-41$ & 111.21 & & $R$ & & & $R$ & $R$ & & 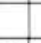 & & & & & & $\mathrm{R}$ & & & $\mathrm{R}$ & & & A & 02 & \\
\hline $14-1,40-41$ & 117.41 & & $R$ & & & $\mathrm{~F}$ & $\mathrm{R}$ & \begin{tabular}{l|l}
$R$ & \\
\end{tabular} & $R$ & & & & & & & & & & & & A & .025 & \\
\hline $14-3, \quad 40-41$ & 120.41 & & $\mathrm{~F}$ & & & $\mathrm{~F}$ & $R$. & $R /$ & $R$ & & & & & & & & & & & & A & 05 & \\
\hline 15-2. $40-41$ & 128.51 & & & & & $\mathrm{R}$ & $\mathrm{R}$ & & & & & & & & & & & & & & A & .025 & \\
\hline $15-4, \quad 40-41$ & 131.51 & & $R$ & & & 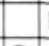 & $R$ & & $R$ & & & & F & $R$ & & & & & & & A & 02 & \\
\hline $15-5, \quad 40-41$ & 133,01 & & $R$ & & & $R$ & & $R \mid R$ & $R$ & & & & $\mathrm{~F}$ & $R$ & & & & & & & A & 02 & \\
\hline $16-1,40-41$ & 136.61 & & & & & $\mathrm{~F}$ & $\mathrm{R}$ & $R$ & & & & & & & & & & & & & C & 05 & \\
\hline $16-2.40-41$ & 138.11 & & $\square$ & & & $R$ & $R$ & $R$ & 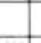 & & & & & & & & & $\mathrm{R}$ & & & A & 025 & \\
\hline $16-4, \quad 40-41$ & 141,11 & & $R$ & & & & $R$ & & $R$ & & & & & & & & & & & & A & 02 & \\
\hline $17-1, \quad 40-41$ & 146.21 & & 1 & & & $R$ & & & $R$ & & & & & & & & & & & & C & .075 & \\
\hline $17-3, \quad 40-41$ & 149.21 & B & \begin{tabular}{l|l}
$R$ & $F$ \\
\end{tabular} & $R$ & & $\mathrm{~F}$ & $R$ & $R$ & $R$ & & & & $R$ & & & & & & & & \begin{tabular}{l|l}
$R$ & $C$ \\
\end{tabular} & 1 & \\
\hline $17-6, \quad 40-41$ & 153. 71 & & $R$ & & & $R$ & & \begin{tabular}{l|l}
$\mathrm{R}$ & $\mathrm{H}$ \\
\end{tabular} & $R$ & & & & & & & & & & & & A & 02 & \\
\hline $18-1,40-41$ & 156.51 & & $R$ & & & $\mathrm{R}$ & $\mathrm{R}$ & & & & & & & F & & & & & & & C & 05 & \\
\hline $18-2, \quad 40-41$ & 157.31 & & $R$ & & & \begin{tabular}{|l|} 
\\
\end{tabular} & $R$ & & & & & & & & & & & & & & A & 02 & \\
\hline $18-5 . \quad 40-41$ & 161.81 & & $R$ & & & $\mathrm{R}$ & $\mathrm{R}$ & $R$ & & & & & & & & & & & & & A & .02 & \\
\hline $19-1.40-41$ & 165.41 & & & & & $R$ & & & $R$ & & & & & & & & & & & & A & .02 & \\
\hline $20-1,40-41$ & 175.01 & & $R$ & & & $F$ & $\mathrm{R}$ & $R$ & & & & & & & & & & & & & C & 05 & \\
\hline $20-4 \cdot \quad 40-41$ & 179.51 & & + & & & $R$ & $R$ & & & & & & & & & & & & & & A & 02 & \\
\hline $20-5 . \quad 40-41$ & 181.01 & & $F$ & & & $F$ & & $R$ & $R$ & & & & & & & & $R$ & & & & C & 05 & \\
\hline $21-1,40-41$ & 184.41 & & $R$ & & & $F$ & $R$ & $R$ & & & & & & & $R$ & & & & & & C & 1 & \\
\hline $21-2 . \quad 40-41$ & 185.91 & & $\mathrm{~F}$ & & & $F$ & $\mathrm{R}$ & \begin{tabular}{l|l} 
\\
$R$
\end{tabular} & $\mathrm{R}$ & & & & & & & & & & & & C & 15 & \\
\hline $22-1, \quad 40-41$ & 193. 81 & & $F$ & & & $F$ & $R$ & & $R$ & & & & & & & & & & & & A & 05 & \\
\hline $22-2 . \quad 40-41$ & 195.31 & & $R$ & & & $F$ & $R$ & & $R$ & & & & & F & & & & & & & A & 05 & \\
\hline $22-2, \quad 40-41$ & 204.71 & & $R$ & & & $R$ & & & & & & & & & & & & & & & A & $0 ?$ & \\
\hline $22-3, \quad 40-41$ & 206.21 & & $R$ & & & $R$ & & & $\mathrm{R}$ & & & & & & & & & & & & C & 02 & \\
\hline
\end{tabular}


Table 4 (continued).

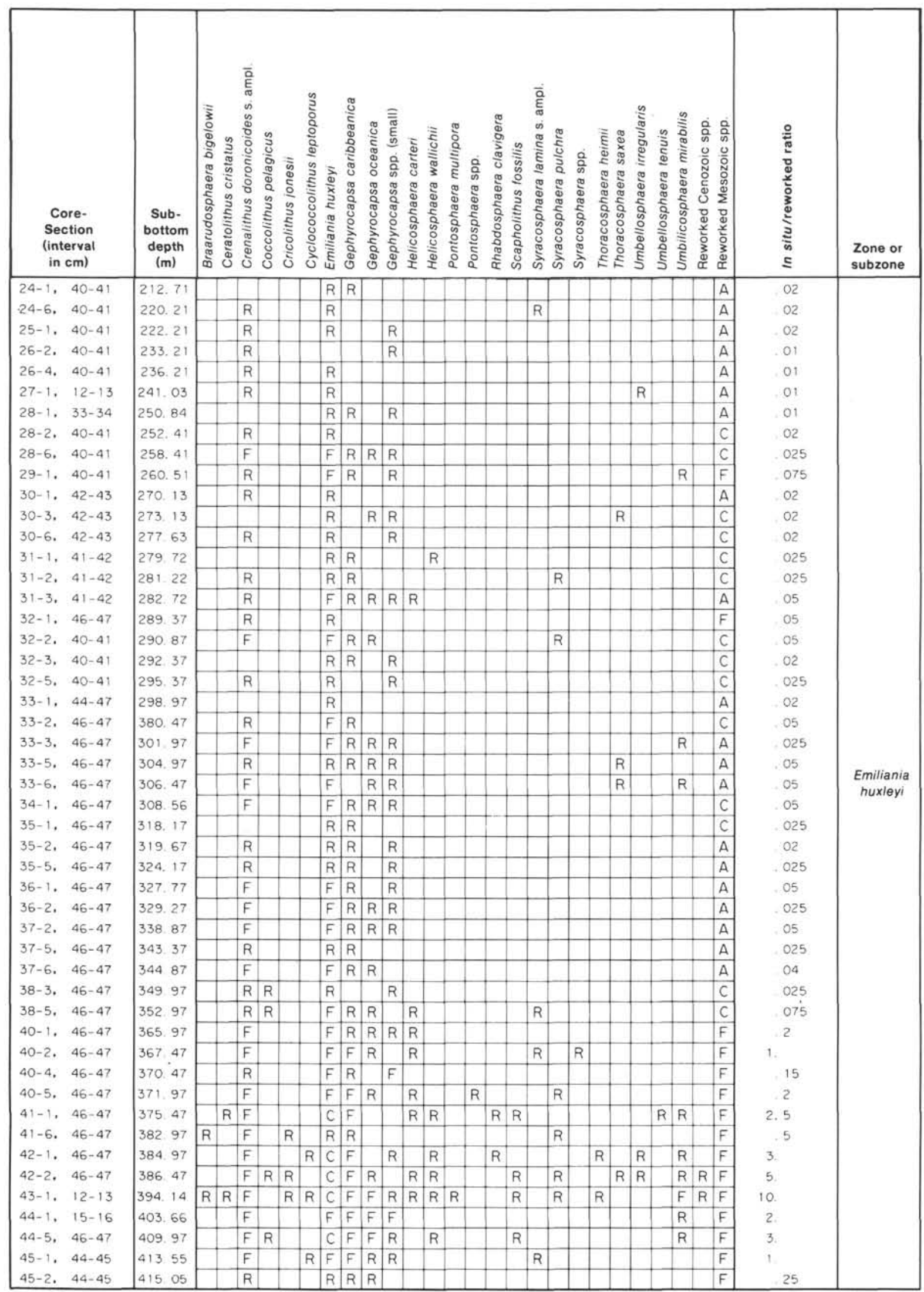

Note: For abundance, $\mathrm{A}=$ abundant, $1-10$ specimens $/$ field: $\mathrm{C}=$ common, 1 specimen $/ 2-10$ fields: $\mathrm{F}=$ few, 1 specimen $/ 11-100$ fields; $\mathrm{R}=$ rare, 1 specimen/101-1000 fields (when viewed at a magnification of $\times 1250$ ). 
Table 5. Distribution of calcareous nannofossils, Site 623.

\begin{tabular}{|c|c|c|c|c|c|c|c|c|c|c|c|c|c|c|c|c|c|c|c|c|c|c|c|c|c|c|c|}
\hline $\begin{array}{c}\text { Core- } \\
\text { Section } \\
\text { (interval } \\
\text { in cm) }\end{array}$ & $\begin{array}{c}\text { Sub- } \\
\text { bottom } \\
\text { depth } \\
(\mathrm{m})\end{array}$ & 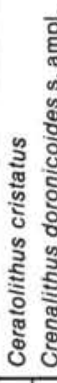 & 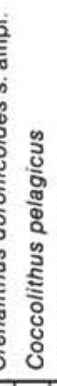 & & 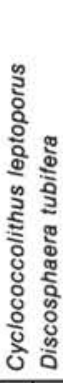 & 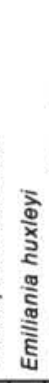 & 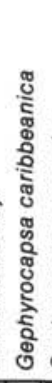 & & & & & 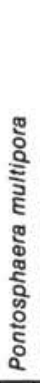 & 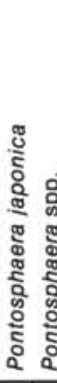 & 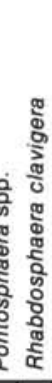 & 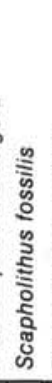 & 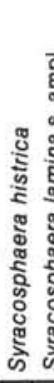 & 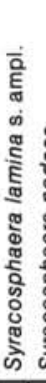 & 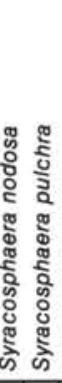 & & 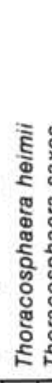 & & 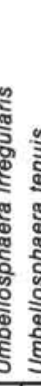 & 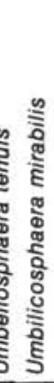 & 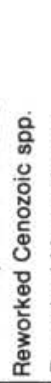 & 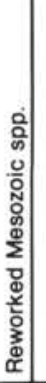 & 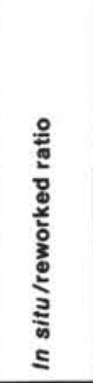 & $\begin{array}{l}\text { Zone or } \\
\text { subzone }\end{array}$ \\
\hline $1-1,40-41$ & 41 & \begin{tabular}{l|l} 
& $R$ \\
\end{tabular} & & & & $R$ & $R$ & & $R$ & \begin{tabular}{l|l}
$R$ \\
\end{tabular} & & & & & & & & $\mathrm{R}$ & $R$ & & $R$ & F & R & & $F$ & .075 & \multirow{49}{*}{$\begin{array}{c}\text { Emiliania } \\
\text { huxleyi }\end{array}$} \\
\hline $1-1,110-111$ & 1. 11 & $R$ & & & & $\mathrm{~F}$ & $\mathrm{R}$ & $R$ & & & $R$ & & & $R$ & & & & & & & & & & & $\mathrm{~F}$ & 1 & \\
\hline $1-2.40-41$ & 1. 91 & $R$ & & & & $R$ & & $R$ & $R$ & & & & & & $R$ & & & & & & & & & & $\mathrm{~F}$ & 1 & \\
\hline $1-3.40-41$ & 3. 41 & R & & & & $\mathrm{R}$ & & & & & & & & & $R$ & & & & & & & & & & C & 05 & \\
\hline 1-4. $40-41$ & 4. 91 & $R$ & & & & $\mathrm{R}$ & $\mathrm{R}$ & $\mathrm{R}$ & & & & & & & & & & & & $\mathrm{R}$ & & & $R$ & & $F$ & 1 & \\
\hline $2-1,40-41$ & 7.01 & $R$ & & & & $\mathrm{~F}$ & $R$ & & & & $R$ & $\mathrm{R}$ & & $R$ & & & $R$ & $R$ & & & $\mathrm{R}$ & & & & $\mathrm{F}$ & 1 & \\
\hline $2-3,40-41$ & 10. 01 & & & & & $\mathrm{R}$ & & & $\mathrm{R}$ & & & & & & & & & & & & & & $R$ & & $R$ & 1. & \\
\hline 2-4. $40-41$ & 11.51 & $\mathrm{~F}$ & & & & $\mathrm{~F}$ & $R$ & & & & & & & & & & & & & & & & $R$ & & C & 05 & \\
\hline 2-6. $40-41$ & 14. 51 & $R$ & & $R$ & & $F$ & & $\mathrm{R}$ & $R$ & & 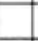 & & & & & & $\mathrm{R}$ & $\mathrm{R}$ & & & & & & & $\mathrm{C}$ & 075 & \\
\hline $3-1,40-41$ & 16. 61 & $\mathrm{R}$ & & $R$ & & $R$ & $R$ & & - & & $R$ & & & & & & & & & & & & & & C & .075 & \\
\hline 3-3. $40-41$ & 19.61 & $R$ & & & & $\mathrm{R}$ & & $\mathrm{R}$ & $R$ & & & & & & & & & & & & & & & & C & .05 & \\
\hline $3-5,40-41$ & 22. 61 & $R$ & & & & $F$ & $R$ & & $R$ & & & & & & & & & & & & & & & & C & .05 & \\
\hline $4-1.40-41$ & 26. 21 & $R$ & & $R$ & & $R$ & $\mathrm{R}$ & $\mathrm{R}$ & & & & & & & & & & & & & & & & & $\mathrm{F}$ & .1 & \\
\hline $4-3,40-41$ & 29.21 & \begin{tabular}{|l|l}
$R$ & $F$ \\
\end{tabular} & $R$ & & & $\mathrm{~F}$ & $\mathrm{~F}$ & $R$ & $R$ & $\mathrm{R}$ & & & & & & & & & & & $\mathrm{R}$ & $2 \mathrm{~F}$ & $\begin{array}{ll}R \\
R\end{array}$ & & $F$ & .4 & \\
\hline $4-5,40-41$ & 32.21 & \begin{tabular}{|l|l} 
& $F$ \\
\end{tabular} & & & & $R$ & $\mathrm{R}$ & & $\mathrm{R}$ & & . & & & & $R$ & & & & & & & F & 2 & & C & .05 & \\
\hline $5-1,40-41$ & 35. 81 & $F$ & & & & $\mathrm{~F}$ & $\mathrm{R}$ & $R$ & & $\mathrm{R}$ & R & & & $R$ & $R$ & & & & & & & & & & C & 25 & \\
\hline $5-3.40-41$ & 38.81 & $R$ & & & & $R$ & $R$ & & 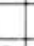 & & & & & $R$ & & & & & & & & & & & $\mathrm{~F}$ & 1 & \\
\hline $5-5,40-41$ & 41.81 & & & & & $R$ & & $\mathrm{R}$ & $R$ & $R$ & & & & & & & & & & & & & & & $F$ & 05 & \\
\hline $6-1.40-41$ & 45.81 & R & & & & $R$ & $R$ & & $R$ & & & & & & & & & & & & & & $R$ & & $\mathrm{C}$ & .05 & \\
\hline $6-3.40-41$ & 48. 41 & $\mathrm{R}$ & & & & $\mathrm{F}$ & $R$ & $\mathrm{R}$ & & $\mathrm{R}$ & & & $\mathrm{R}$ & & & & $R$ & $R$ & $R$ & & & & & & $C$ & 075 & \\
\hline $6-5.40-41$ & 51.41 & $F$ & & & & $R$ & $R$ & & & & & & & & & & & & & & & & & & C & 025 & \\
\hline $7-1,40-41$ & 55. 01 & & & & & $\mathrm{~F}$ & & $\mathrm{R}$ & & & & & & & & & & & & & & & $R$ & & $\mathrm{C}$ & 05 & \\
\hline 7-2. $40-41$ & 56.51 & $\mathrm{R}$ & & & $R$ & $\mathrm{~F}$ & $\mathrm{R}$ & & $R$ & & $\mathrm{R}$ & & & & & & & & & & & & & & C & 1 & \\
\hline $7-3.40-41$ & 58. 01 & R & & $\mathrm{R}$ & $R$ & $\mathrm{~F}$ & $\mathrm{R}$ & & & & & & & & & & & & & & & & & & $\mathrm{C}$ & .05 & \\
\hline $8-1.52-53$ & 64.73 & & & & & $\mathrm{~F}$ & & $\mathrm{R}$ & $R$ & & & & & & & & & & & & & & & & $\mathrm{C}$ & .05 & \\
\hline $8-2,78-79$ & 66. 49 & & & & & $R$ & $\mathrm{R}$ & & & & & & & & & & & $R$ & & & & & $\mathrm{R}$ & $R$ & A & .025 & \\
\hline $8-3,50-51$ & 67.71 & $R$ & & & & $R$ & $\mathrm{R}$ & & $R$ & & & & & & & & & & & & & & $\mathrm{R}$ & & C & .025 & \\
\hline $9-2.49-50$ & 75.80 & R & & & & & $R$ & & & $\mathrm{R}$ & & & & & & & & & & & & & & & C & .025 & \\
\hline $9-3.49-50$ & 77.30 & & & & & $R$ & $R$ & & $R$ & & & & & & & & & $\mathrm{R}$ & & & & & & $R$ & $C$ & 05 & \\
\hline 9-4. $49-50$ & 78.80 & $R$ & & & & $R$ & $\mathrm{R}$ & $R$ & & & & & & & $R$ & & & & & & & & & & C & .05 & \\
\hline $10-1.55-56$ & 83. 96 & & & & & $\mathrm{R}$ & & $\mathrm{R}$ & & & & & & & & & & & & & & & & & C & .025 & \\
\hline $10-3,60-61$ & 87.01 & $R$ & & & & $\mathrm{R}$ & & & $R$ & & & & & & & & & & & & & & & & C & .05 & \\
\hline $10-4, \quad 10-11$ & 88.01 & $R$ & & & & & $\mathrm{R}$ & & & & & & & & & & & & & & & & & & $\mathrm{C}$ & 05 & \\
\hline $11-1,26-27$ & 93. 26 & & & & & $\mathrm{~F}$ & \begin{tabular}{|l|l}
$R$ \\
\end{tabular} & $\mathrm{R}$ & & & & & & & & $R$ & & & & & & & & & $\mathrm{~F}$ & -1 & \\
\hline $11-3.40-41$ & 96.41 & & & & & $\mathrm{R}$ & $R$ & & $R$ & & & & & & & & & $R$ & & & & & & & $\mathrm{C}$ & 05 & \\
\hline $11-4,28-29$ & 97.79 & F & & & & $R$ & & $\mathrm{R}$ & & & & & & & & & & & & & & & & & $\mathrm{C}$ & 025 & \\
\hline $12-1,45-46$ & 103,06 & \begin{tabular}{l|l}
$R$ & $F$ \\
\end{tabular} & & $\mathrm{R}$ & \begin{tabular}{l|l}
$R$ & $R$ \\
\end{tabular} & $\mathrm{C}$ & $R$ & $\mathrm{~F}$ & $R$ & $R$ & $R$ & $R$ & \begin{tabular}{l|l}
$R$ & $R$ \\
\end{tabular} & $R$ & $R$ & & & & & & R & & $R$ & & $\mathrm{C}$ & 1. & \\
\hline $12-3.29-30$ & 105.89 & \begin{tabular}{l|l}
$R$ & $F$ \\
\end{tabular} & & & & $F$ & $\mathrm{R}$ & & $R$ & & & & & $R$ & $R$ & & & & & $\mathrm{R}$ & $R$ & & & & C & .25 & \\
\hline $12-4,21-22$ & 107.31 & $F$ & & & & $\mathrm{R}$ & $R$ & $R$ & & $R$ & $R$ & & & & & & & & & $R$ & & & & & C & .05 & \\
\hline $13-1,26-27$ & 112.26 & $F$ & & & & C & $R$ & $R$ & & & $R$ & & $R$ & & & & & & & $R$ & & & $\mathrm{R}$ & & C & 2 & \\
\hline $14-1.41-42$ & 121.82 & c & $R$ & & & C & $\mathrm{F}$ & $\mathrm{R}$ & $R$ & $\mathrm{R}$ & $R$ & & & $R$ & $R$ & & $R$ & & $\mathrm{R}$ & & & & $R$ & $R$ & A & 2 & \\
\hline $14-2, \quad 41-42$ & 123.32 & $F$ & & & & C & $R$ & $\mathrm{R}$ & $R$ & $\mathrm{R}$ & & & & & $R$ & & & $R$ & & & & & $R$ & & $\mathrm{C}$ & 2 & \\
\hline $14-3,48-49$ & 124.89 & $F$ & & & & C & & $R$ & & & & & & $R$ & & & $R$ & & & & R & $R / F$ & \begin{tabular}{l|l}
$\mathrm{R}$ & $\mathrm{B}$ \\
\end{tabular} & & A & 15 & \\
\hline $15-1,26-27$ & 131.06 & F & & & & $F$ & $R$ & & $R$ & & & & & & & & & & & & & & & & C & 05 & \\
\hline $16-1,40-41$ & 140.51 & F & & & & $R$ & $R$ & & & & & & & & & & & & & & & & & & C & .05 & \\
\hline $16-2 . \quad 40-41$ & 142.01 & F & & & & $F$ & $R$ & $R$ & & $R$ & & & & & & & & & & & & & & & A & 05 & \\
\hline $16-3,40-41$ & 143.51 & F & & & & $\mathrm{R}$ & & & $R$ & $\mathrm{R}$ & & & & & & & & & & & & f & $R$ & & A & 02 & \\
\hline $17-1,40-41$ & 149. 81 & $F$ & & & & $F$ & $R$ & $R$ & & $R$ & $\mathrm{R}$ & & & & $R$ & & & & & $\mathrm{R}$ & $R$ & & $R$ & & C & 2 & \\
\hline $17-2, \quad 40-41$ & 151.31 & F & & & & $\mathrm{F}$ & & $\mathrm{R}$ & R & $R$ & & & & & & & & & & $\mathrm{R}$ & & & & & C & 1 & \\
\hline
\end{tabular}

Note: For abundance, $A=$ abundant. $1-10$ specimens/field: $C=$ common, 1 specimen $/ 2-10$ fields: $F=$ few. 1 specimen $/ 11-100$ fields: $R=$ rare, 1 specimen/101-1000 fields (when viewed at a magnification of $\times 1250$ ). 
Table 6. Distribution of calcareous nannofossils, Site 624 .

\begin{tabular}{|c|c|c|c|c|c|c|c|c|c|c|c|c|c|c|c|c|c|c|c|c|c|c|c|c|c|c|c|c|c|c|c|c|c|c|c|}
\hline $\begin{array}{l}\text { Core- } \\
\text { Section } \\
\text { (interval } \\
\text { in } \mathrm{cm} \text { ) }\end{array}$ & \begin{tabular}{|c|}
$\begin{array}{c}\text { Sub- } \\
\text { bottom } \\
\text { depth } \\
\text { (m) }\end{array}$ \\
\end{tabular} & 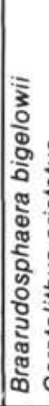 & & 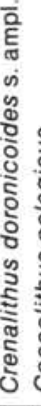 & 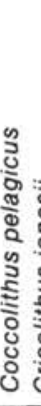 & & 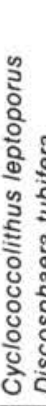 & & & 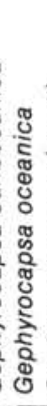 & & 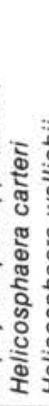 & & & 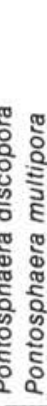 & 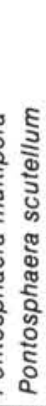 & & & 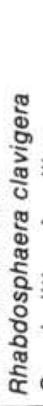 & & 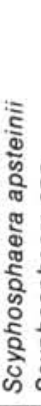 & & & 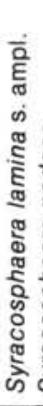 & & & & 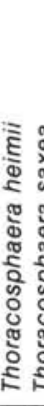 & & 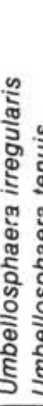 & & 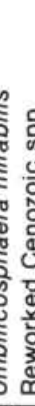 & & 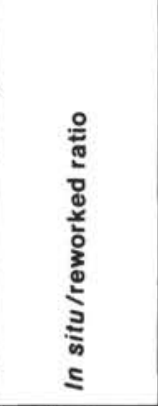 & $\begin{array}{l}\text { Zone or } \\
\text { subzone }\end{array}$ \\
\hline $1-1,6-7$ & .07 & & F & $\overline{F F}$ & \begin{tabular}{l|l}
$R$ & $F$ \\
\end{tabular} & $\bar{F}$ & \begin{tabular}{l|l}
$C$ & $R$ \\
$C$
\end{tabular} & $2 v$ & $F$ & C & $F$ & C & \begin{tabular}{l|l}
$C$ & $R$ \\
\end{tabular} & $\begin{array}{ll}R & R \\
\end{array}$ & $\begin{array}{l}\mathrm{R} \\
\mathrm{R}\end{array}$ & $\mathrm{R}$ & & $\mathrm{R}$ & $F$ & $F$ & $\mathrm{R}$ & \begin{tabular}{|l|l|}
$R$ \\
\end{tabular} & F & $F$ & F & $\overline{F P}$ & $F \mid F$ & $\overline{F F}$ & $F$ & \begin{tabular}{l|l}
$F$ & $F$
\end{tabular} & 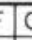 & 7 & $R$ & 6000. & \multirow{47}{*}{$\begin{array}{c}\text { Emiliania } \\
\text { huxleyi }\end{array}$} \\
\hline $1-1,14-15$ & 15 & \begin{tabular}{|l|l}
$R$ & $R$ \\
\end{tabular} & $\mathrm{~F} F$ & $\mathrm{FF}$ & $R F$ & $\mathrm{~F}$ & $C R$ & A & c & c & $\mathrm{F}$ & $\mathrm{F}$ & $\mathrm{F} R$ & 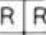 & $\begin{array}{l}\mathrm{R} \\
\mathrm{R}\end{array}$ & & & & $\mathrm{F}$ & $F$ & & $R$ & $\mathrm{~F}$ & $\mathrm{~F}$ & $\mathrm{~F}$ & $\mathrm{~F} F$ & $\mathrm{~F}$ & $\mathrm{~F}$ & $\mathrm{~F}$ & $R / F$ & 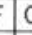 & F & $R$ & 4000 & \\
\hline $1-1,25-26$ & .25 & & & & & & & & & & & & & & & & & & & & & & & & & & & & & & & & $\mathrm{R}$ & Barren & \\
\hline $1-1.43-44$ & 44 & & & $\mathrm{R}$ & & & & $\mathrm{F}$ & $\mathrm{F}$ & $R$ & $R$ & & & & & & & & & & & & & . & & . & & & & & & & c & .05 & \\
\hline $1-2.43-44$ & 1. 94 & & R $\mathrm{F}$ & $\mathrm{F}$ & & R F $F$ & R & C & $F$ & $R$ & $R$ & $R$ & $\mathrm{R}$ & & & & & & $R$ & $R$ & & & & $R$ & & R $P$ & $R F$ & $R F$ & R & F & F & & $\mathrm{F}$ & 5 & \\
\hline $1-3,43-44$ & 3. 44 & & & $R$ & & $\mathrm{R}$ & & $F$ & $F$ & O & $R$ & $R$ & & & & & & & & $R$ & & & & $R$ & & & $R$ & & & & F & & $\mathrm{F}$ & 5 & \\
\hline $1-5,12-13$ & 6. 13 & & & $\mathrm{~F}$ & & & & C & $F$ & $R$ & $R$ & $R$ & $R$ & & & & & & . & & & & $R$ & & & & & & & & & & C & 25 & \\
\hline $2-1,40-41$ & $\begin{array}{ll}731 \\
\end{array}$ & & $\mathrm{R} F$ & $F$ & & & & C & $R$ & $\mathrm{~F}$ & $R$ & $R$ & $R$ & & & & & $R$ & $R$ & & & & & & & & & & & & & & $C$ & 5 & \\
\hline 2-2. $40-41$ & 881 & & & $\mathrm{R}$ & & & & $F$ & $R$ & $R$ & $R$ & if & $R$ & & & & & & & & & & & & & & & & & $R$ & F & 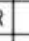 & $C$ & 15 & \\
\hline $2-3.40-41$ & 1031 & & & $F \mid F$ & $\mathrm{R}$ & & & $\mathrm{F}$ & & $R$ & $R$ & $R$ & & & & & & & & & & & & & & & & $R$ & & $R$ & & & $\mathrm{~F}$ & 2 & \\
\hline $2-5,40-41$ & 1331 & & & $R$ & & & & $\mathrm{~F}$ & $R$ & $R$ & & & . & & & & & & & & & & & & & & & & & & & & $C$ & .05 & \\
\hline $3-1,40-41$ & 16.91 & & & $\mathrm{~F}$ & & $\mathrm{R}$ & & $F$ & $R$ & $R$ & $R$ & & . & & & & & & $R$ & $R$ & & & & & & $\mathrm{R}$ & & & & & F & ? & $\mathrm{F}$ & 15 & \\
\hline $3-2,40-41$ & $18 \quad 41$ & & & $\mathrm{R}$ & & & $R$ & $F$ & $R$ & $\mathrm{R}$ & & . & - & & & & & & $R$ & & & & & & & $R$ & & & & & F & s. & C & 5 & \\
\hline $3-4,40-41$ & 21.41 & & $\mathrm{R} F$ & $\mathrm{~F}$ & & R $\mathrm{F}$ & $\mathrm{R}$ & C & $F$ & $R$ & & $R$ & $R$ & & & & $R$ & & & & & & & & $\mathrm{R}$ & $R$ & & & & & & & $\mathrm{~F}$ & .1 & \\
\hline $3-5,40-41$ & 2291 & $R$ & & R F $F$ & $\mathrm{R}$ & & & $\mathrm{F}$ & & $R$ & $R$ & & & & & & & & & & & & & & & & & & & & & & C & .1 & \\
\hline $4-1,40-41$ & 2651 & $R$ & & $R$ & & & & $\mathrm{~F}$ & $R$ & $R$ & & & $R$ & & & & & & & & & & & & & & & & & & & & C & 1 & \\
\hline $4-2,40-41$ & 2801 & & & $R$ & & & & $\mathrm{~F}$ & $R$ & $R$ & $R$ & $R$ & & & & & & & & $R$ & & & & & & & & & & & f & & $\mathrm{F}$ & 15 & \\
\hline $4-4,40-41$ & 3101 & & & $R$ & & & & $\mathrm{~F}$ & $R$ & $R$ & & & & & & & & & & $R$ & & & & $R$ & & & & & & & & & C & .05 & \\
\hline $4-5, \quad 40-41$ & 32.51 & & & $R$ & & & & $F$ & $R$ & $R$ & & $R$ & & & & & & & & & & & & & & & & & & & & & C & .05 & \\
\hline $5-1,40-41$ & 36.11 & & & $\mathrm{R}$ & & & & $R$ & & & & $R$ & $\mathrm{R}$ & & & & & & & & & & & & & & & & & & & & C & .025 & \\
\hline $5-2.40-41$ & 37.51 & & & & & & & $R$ & & $R$ & $R$ & & $R$ & & & & & & & $R$ & & & & & & & & & & & & & C & .025 & \\
\hline $5-4,40-41$ & 40.61 & & & $\mathrm{R}$ & & $\mathrm{R}$ & & $\mathrm{F}$ & & & & & & & $R$ & & & & & & & & & & & & & & $\mathrm{R}$ & & & & A & .02 & \\
\hline $5-5,40-41$ & 42.11 & & & $\mathrm{R}$ & & & & $R$ & $R$ & $R$ & & & & & & & & & & & & & & & & & & & $R$ & & F & & C & .025 & \\
\hline $6-1,40-41$ & 45.71 & & & $\mathrm{R}$ & & & & $R$ & & $R$ & & & & & & & & & & & & & & & & & $\mathrm{R}$ & & & & & & $C$ & .05 & \\
\hline $6-2, \quad 40-41$ & 47.21 & & & $\mathrm{R}$ & & & & $R$ & $R$ & & $R$ & & & & & & & & $R$ & & & & & & & & & & & & & & C & .05 & \\
\hline $6-4,40-41$ & $\begin{array}{lll}50 & 21\end{array}$ & & & $R$ & & & & $\mathrm{~F}$ & $R$ & $R$ & & $R$ & & & & & & & & & & & & & & & & $\mathrm{R}$ & & F & 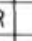 & & A & .05 & \\
\hline $7-1,40-41$ & $\begin{array}{lll}55 & 31\end{array}$ & & & $R$ & & & & $R$ & & & & & & & & & & & & & & & & & & & & & & & & & A & .02 & \\
\hline $7-2,40-41$ & 56.81 & & & $\mathrm{R}$ & & & & $\mathrm{R}$ & & & $R$ & & $R$ & & & & & & & & & & & & & $R$ & $\mathrm{R}$ & & & F & R F & ? & C & .05 & \\
\hline $8-1,40-41$ & 6491 & & & . & & & & $R$ & $R$ & & $\mathrm{R}$ & $R$ & & & & & & & & & & & & & & & & & & & & & $\mathrm{~F}$ & .05 & \\
\hline $8-2 \cdot 40-41$ & 66.41 & & & $R$ & & & & & $R$ & & $\mathrm{R}$ & & & & & & & & & & & & & & & & & & & & & & C & .025 & \\
\hline $9-1,40-41$ & 7451 & & & $\mathrm{R}$ & & & & $\mathrm{R}$ & & $R$ & & & & & & & & & & $R$ & & & & & $R$ & & & & & & & & C & .05 & \\
\hline $9-2,40-41$ & $\begin{array}{lll}76 & 01\end{array}$ & & & $\mathrm{R}$ & & & & $\mathrm{R}$ & & & & & & & & & & & & & & & & & & & & & & & & & A & .02 & \\
\hline $9-4, \quad 40-41$ & 7901 & & & . & & $R$ & & & $R$ & & & & & & & & & & & & & & & & & & & & & $R F$ & 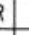 & & C & .04 & \\
\hline $10-1,40-41$ & 84.11 & & & $R$ & & & & R & & & & & & & & & & & & & & & & & & & $R$ & & & & & & $F$ & .025 & \\
\hline $11-1,56-57$ & 93. 87 & & & $R$ & & & & $R$ & & $R$ & & & & & & & & & & & & & & & & & & & & & & & C & .02 & \\
\hline $11-2.20-21$ & 9501 & & & $R$ & & $R$ & & $R$ & & & $R$ & & & & & & & & & & & & & & & & & & & & & & C & .025 & \\
\hline $12-1,40-41$ & 103.11 & & & $\mathrm{R}$ & & $\mathrm{R}$ & & $R$ & & $R$ & & & & & & & & & & & & & $R$ & & $R$ & & & & & $R$ & & & A & .04 & \\
\hline $12-2 \cdot \quad 35-36$ & 104.56 & & & $\mathrm{R}$ & & & & $\mathrm{F}$ & & $R$ & $R$ & & & & & & $\mathrm{R}$ & & & & & & & $R$ & & & & & & & F & & $C$ & .1 & \\
\hline $12-3.40-41$ & 106.11 & & & $R$ & & & $\mathrm{R}$ & $\mathrm{F}$ & $R$ & $R$ & & & & $\mathrm{R}$ & & & & $R$ & & & & & & & & & & & & $R$ & & & C & .1 & \\
\hline $14-1 . \quad 43-44$ & 118.23 & & & & & & & $R$ & $R$ & & $R$ & $R$ & & & & $\mathrm{R}$ & & & & & & & & & & & & & & & & F & A & .025 & \\
\hline $15-1,45-46$ & 127.56 & & & & $\mathrm{R}$ & & & $R$ & $R$ & & & & $R$ & & & & & & & & & & & & & & & & & & & & C & 1 & \\
\hline $15-2 . \quad 45-46$ & 129.06 & & & 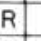 & & & & $R$ & $R$ & & & & & & & & & & & & & & & & & & & & & & & & A & .01 & \\
\hline $15-3 \cdot 34-35$ & 130.44 & & & $\mathrm{R}$ & & & & $\mathrm{R}$ & & $R$ & $R$ & & & & & & & & $R$ & & & & & & & & & & $R$ & & & & C & .025 & \\
\hline $16-1, \quad 43-44$ & 136.84 & & & & & & & $\mathrm{R}$ & $R$ & & & & & & & & & & & & & & & & & & & & & & & & A & .01 & \\
\hline $16-2, \quad 43-44$ & 138.39 & & & $\mathrm{~F}$ & & & & $\mathrm{R}$ & $R$ & $R$ & & & & & & & & & & & & & & & & & & $\mathrm{R}$ & & & & & C & .025 & \\
\hline $17-1.41-42$ & 146.12 & & & $R$ & & $\mathrm{R}$ & & $\mathrm{F}$ & $R$ & & $R$ & & & & & & & & $R$ & & & & & & & & & & & F & & & c & .05 & \\
\hline $18-1,5-6$ & 155.15 & & & & & & & $R$ & $R$ & & $\mathrm{R}$ & & & & & & & & & & & & & & & & & & & & & & C & .025 & \\
\hline
\end{tabular}

Note: For abundance, $V=$ very abundant, $10-100$ specimens/field; $A=$ abundant, $1-10$ specimens $/$ field; $C=$ common, 1 specimen $/ 2-10$ fields; $F=$ few, 1 specimen/11-100 fields; $R=$ rare, 1 specimen/101-1000 fields (when viewed at a magnification of $\times 1250$ ).

high deposition rates in this region. A sedimentation rate of $2.1 \mathrm{~cm} / 1000 \mathrm{yr}$. is calculated for the Holocene; a rate of nearly $7.5 \mathrm{~m} / 1000 \mathrm{yr}$. for the late Wisconsin glacial is based on projections to seismic Horizon " 30 " (introductory chapter, this volume).

\section{Site 615}

Site $615\left(25^{\circ} 13.34^{\prime} \mathrm{N}, 85^{\circ} 59.53^{\prime} \mathrm{W}\right.$; water depth, $3284 \mathrm{~m}$ ) is located approximately $40 \mathrm{~km}$ south of Sites 623 and 624 . It is positioned adjacent to the channel on 
the distal part of the lower fan. This site, the deepest drilled on Leg 96, was cored to a depth of $523.2 \mathrm{~m}$ subbottom. Thick sands and silt-mud sequences as old as the early Wisconsin glacial (Ericson Zone W) were recovered (Site 615 chapter, this volume). The Wisconsin interstadial (Ericson Zone X) is represented by a $29-\mathrm{m}$ thick carbonate sequence, thought to have originated on the West Florida platform (Brooks et al., this volume).

The upper $17 \mathrm{~cm}$ of sediment recovered in Section 615-1-1 consists of a marly foraminiferal ooze containing abundant, moderately well-preserved calcareous nannofossils typical of pelagic Holocene sediments in the Gulf of Mexico (Table 7). Emiliania huxleyi is the dominant taxon.

The interval below this ooze, from Samples 615-1-1, $32 \mathrm{~cm}$ through $615-48, \mathrm{CC}$, is a late Wisconsin glacial interbedded sand-mud sequence in which reworked Cretaceous nannofossils are the major component. Only very slight to slight increases in the indigenous Pleistocene flora are encountered in this interval. The increase found in Core 615-18 (153 m sub-bottom) may be correlative with the increase in the bottom sample of Hole 614A (shown in Fig. 4 as line B). Although rare, $E$. huxleyi is clearly the dominant Quaternary taxon, placing the entire upper interval from sea bottom through Sample 615$48, C C$ in the E. huxleyi Acme Zone of Gartner and Emiliani. SEM examinations of Samples $615-47, C C, 615-$ $46, \mathrm{CC}, 615-42-1,3-4 \mathrm{~cm}$, and other samples near the bottom of this interval confirm that within the indigenous component, E. huxleyi is dominant over Gephyrocapsa spp.

A calcareous nannofossil-foraminiferal ooze deposited during the middle Wisconsin interstadial occurs in Samples $615-48, C C$ through $615-51, C C$. This interval appears to grade from a nannofossil ooze in Core 615-49 into a foraminiferal ooze in Core $615-51$ and is terminated by a gravelly conglomerate in Sample 615-51,CC. Reworked Eocene to early Pleistocene taxa are common in the flora in this interval, including important biostratigraphic marker species such as Pseudoemiliania lacunosa, Cyclococcolithus macintyrei, Discoaster brouweri, D. pentaradiatus, Sphenolithus abies, Reticulofenestra pseudoumbilica, D. quinqueramus, and $D$. barbadiensis. Within the highly abundant late Pleistocene flora in this interval, Gephyrocapsa spp. appears dominant over $E$. huxleyi, placing these and remaining sediments in the $E$. huxleyi Zone of Gartner and Emiliani. Although E. huxleyi is abundant, SEM studies of samples from this carbonate ooze show that $E$. huxleyi is a relatively minor component of the Pleistocene assemblage. Gephyrocap$s a$ spp. is clearly the dominant taxa. The last occurrence of the planktonic foraminifer Globorotalia flexuosa also occurs in this interval (Site 615 chapter, this volume). This gives secondary support for the placement of the boundary between the $E$. huxleyi Zone and the $E$. huxleyi Acme Zone in Sample 615-48,CC. The lowest dominant occurrence of $E$. huxleyi has been shown to occur at 85,000 yr. ago in tropical and subtropical waters (Thierstein et al., 1977). At Site 619, this event has been dated at approximately 84,000 yr. ago (see Site 619 , this chapter).
Below the conglomerate in Sample 615-51,CC, a pelagic foraminiferal ooze containing an abundant late Pleistocene assemblage was recovered in Section 615-52-1. This ooze contains relatively few reworked Pliocene taxa and appears to have been deposited by hemipelagic sedimentation. Seismic Horizon "30" (introductory chapter, this volume) is thought to be associated with this carbonate unit. The regional distribution of Horizon " 30 " may be the result of widespread hemipelagic sedimentation in response to the rise of sea level during the middle Wisconsin interstadial.

A dark brown mud containing a sparse nannofossil assemblage is encountered in Sample 615-52,CC. This interval is again dominated by reworked Cretaceous taxa indicating a return to terrigenous sedimentation.

\section{Site 614}

Site $614\left(25^{\circ} 04.08^{\prime} \mathrm{N}, 86^{\circ} 08.21^{\prime} \mathrm{W}\right.$; water depth, $3314 \mathrm{~m}$ ) was drilled $23 \mathrm{~km}$ southwest of Site 615 in an area near the termination of the lower fan channel. Holes 614 and 614A together penetrated to a total depth of $150.3 \mathrm{~m}$ sub-bottom. The sand, silt, and mud recovered are similar to the sediments recovered in the upper portions of Site 615 (Site 614 chapter, this volume) and are interpreted to be in the Emiliania huxleyi Acme Zone of Gartner and Emiliani.

The marly foraminiferal ooze recovered in Section 614 $1-1$ is the only interval that contains abundant Quaternary nannofossils (Table 8). The dominant taxon in this assemblage is $E$. huxleyi.

Reworked Cretaceous nannofossils are the major constituent of the remainder of Holes 614 and 614A. Because of the rapid sedimentation rate (calculated at $6.4 \mathrm{~m} /$ 1000 yr. based on correlations with seismic Horizon “30"), only rare Pleistocene nannofossils are present in the samples. A slight increase in the indigenous Pleistocene taxa is observed in Sample 614A-13,CC (Fig. 4).

\section{Fan Margin Site}

One site was selected on the fan margin to core the feature identified as a slump by Walker and Massingill (1970; Fan Margin Introduction and Summary, this volume).

\section{Site 616}

Site $616\left(26^{\circ} 48.67^{\prime} \mathrm{N}, 86^{\circ} 52.83^{\prime} \mathrm{W}\right.$; water depth $2999 \mathrm{~m}$ ) was cored to a total sub-bottom depth of $371 \mathrm{~m}$. This site is located $55 \mathrm{~km}$ northeast of the midfan channel (Fig. 1). Muds, sands, and fining-upward sand-siltmud sequences were recovered. The upper $96 \mathrm{~m}$ consist of a mud and silt unit displaying dips of up to $65^{\circ}$ that is interpreted to be a series of discrete slides (Site 616 chapter, this volume). Excluding this interval, sedimentation rates of the late Wisconsin glacial sediments based on correlations with seismic Horizon " 30 " are as high as $5.6 \mathrm{~m} / 1000 \mathrm{yr}$.

This rapid deposition rate extensively diluted the indigenous Quaternary nannofossil assemblage with reworked Cretaceous taxa (Table 9). The rarity of indigenous taxa makes the determination of the presence of an Emiliania huxleyi/Gephyrocapsa dominance reversal dif- 
Table 7. Distribution of calcareous nannofossils, Site 615.

\begin{tabular}{|c|c|c|c|c|c|c|c|c|c|c|c|c|c|c|c|c|c|c|c|c|c|c|c|c|c|c|c|c|c|c|c|c|c|}
\hline $\begin{array}{l}\text { Core- } \\
\text { Section } \\
\text { (interval } \\
\text { in cm) }\end{array}$ & $\begin{array}{c}\text { Sub- } \\
\text { bottom } \\
\text { depth } \\
\text { (m) }\end{array}$ & 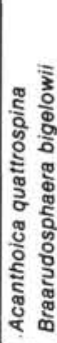 & & 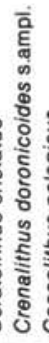 & & 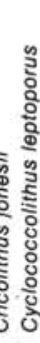 & & & 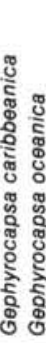 & & & & & & & & 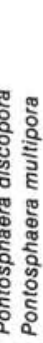 & 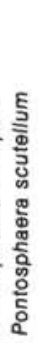 & & & & & & & & & & & 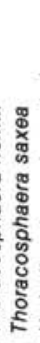 & & & & 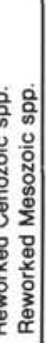 \\
\hline $1-1,23-24$ & 24 & \begin{tabular}{l|l}
$R$ & $R$ \\
\end{tabular} & $F$ & $\mathrm{R} / \mathrm{F}$ & $\begin{array}{l}R \\
R\end{array}$ & $=\mathrm{C}$ & \begin{tabular}{|l|l}
$R$ & \\
\end{tabular} & $A \mid F$ & \begin{tabular}{l|l|l}
$F$ & $F$
\end{tabular} & & $F$ & \begin{tabular}{l|l|}
$R$ &
\end{tabular} & $\mathrm{R}^{\mathrm{R}}$ & & $\mathrm{R}$ & $R$ & & $R$ & & $|F|$ & C & & $\mathrm{R} / \mathrm{R}$ & $\begin{array}{l}R \\
R\end{array}$ & R & $\mathrm{F}$ & & $R$ & $F$ & \begin{tabular}{|l|l}
$R$ & $F$
\end{tabular} & 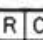 & \begin{tabular}{l|l}
$C$ & $R$
\end{tabular} & 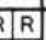 \\
\hline $1-1,50-51$ & .51 & & & $\mathrm{R}$ & $\mathrm{R}$ & ( & & $\mathrm{F}$ & $\mathrm{R}$ & s & $\mathrm{R}$ & & & $R$ & & & & & & $R$ & $\mathrm{R}$ & & & & & R & & R & $R$ & & $\mathrm{R}$ & & c \\
\hline $1-1,74-75$ & .75 & & & $\mathrm{R}$ & & $R$ & & $F$ & & & $R$ & $R$ & & & & & & & & & & & & & & & $\mathrm{R}$ & & $\mathrm{R}$ & & $\mathrm{R}$ & & C \\
\hline $1-2.71-72$ & 2. 22 & & & $\mathrm{R}$ & $R$ & (. & & \begin{tabular}{l|l}
$R$ & $F$ \\
\end{tabular} & $\mathrm{R}$ & & $R$ & & & & & & & & & $R$ & 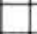 & & & 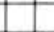 & t & 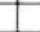 & 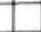 & 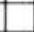 & O & 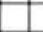 & 1 & & $F$ \\
\hline $2-1,47-48$ & 3. 08 & & & $R$ & & & & \begin{tabular}{l|l} 
& $F$ \\
\end{tabular} & \begin{tabular}{l|l}
$R$ & $R$ \\
$R$
\end{tabular} & s & $R$ & & & & & & & & & & 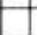 & & & & & & R & & $R$ & & 1 & & c \\
\hline $2-3 . \quad 15-16$ & 5,76 & & & $R$ & $R$ & $R$ & & $\mathrm{~F}$ & & & $R$ & & & & & & & & & & $R$ & & & & $\mathrm{R}$ & $R$ & & & & & $\mathrm{R}$ & & $A$ \\
\hline 2-4. $39-40$ & 750 & & & $F$ & & $R$ & & $F$ & $R$ & q & O & $R$ & & & & & & & & $R$ & & & & & & & $R$ & & & & 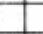 & & A \\
\hline $2-5,9-10$ & 8. 69 & & & \begin{tabular}{l|l}
$F$ & $F$ \\
\end{tabular} & \begin{tabular}{l|l}
$R$ & $R$ \\
\end{tabular} & | & & $R$ & & & $R$ & & & & & & & & $R$ & $R$ & & & & $R$ & & & $R$ & & & & \begin{tabular}{l|l}
$R$ & $R$ \\
\end{tabular} & R & $A$ \\
\hline $2-7 . \quad 16-17$ & 11.77 & & & \begin{tabular}{|l|l|}
$F$ \\
\end{tabular} & & & & $F$ & & & $R$ & & & & & & & & & & & & & & $R$ & & $R$ & & & T & 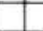 & & c \\
\hline $3-1.34-35$ & 12. 44 & & & \begin{tabular}{l|l}
$F$ \\
$F$
\end{tabular} & $\mathrm{R}$ & 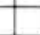 & & $\mathrm{F} / \mathrm{F}$ & R & & $R$ & $R$ & & & & & & & & 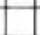 & & & & & $R$ & & $R$ & & & \begin{tabular}{|l|l|}
$R$ & \\
\end{tabular} & $\mathrm{R}$ & R & c \\
\hline $3-3, \quad 42-43$ & 15.53 & & & \begin{tabular}{|l|l|}
$R$ \\
\end{tabular} & & $R$ & & $\begin{array}{ll}R & F \\
\end{array}$ & R & & & & & & & & & & & & & & & & & & & & & & $\begin{array}{ll}\mathrm{R} & \mathrm{R} \\
\end{array}$ & R & $F$ \\
\hline $3-5.42-43$ & 18.53 & & & & & & & & & & 7 & & & & & & & & & 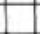 & & & & & & & & & & & & & $R$ \\
\hline $4-1,77-78$ & 20.88 & & & $\mathrm{~A}$ & & & & $F$ & & & $R$ & & & & & & & & & 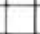 & & & & & $R$ & & $R$ & & & & 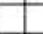 & & c \\
\hline 4-3. $20-21$ & 23. 30 & & & $\mathrm{R}$ & & & & $R$ & & & & & & & & & & & & 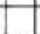 & & & & & & 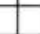 & & & & . & 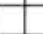 & & $\mathrm{R}$ \\
\hline 4-4. $20-21$ & 2480 & & & $R$ & & & & \begin{tabular}{l|l}
$R$ & $F$ \\
\end{tabular} & $\mathrm{R}$ & & $\square$ & & & & & & & & & $R$ & & & & 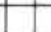 & & 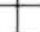 & , & & & 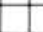 & & 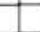 & $F$ \\
\hline $5-1,117-118$ & 30.78 & & & $\mathrm{R}$ & & & & F $F$ & $R$ & & $R$ & & & & & & & & & & & & & & & 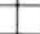 & 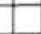 & & & 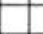 & $\mathrm{A}$ & $R$ & $F$ \\
\hline $5-2,77-78$ & 31.88 & & & $F$ & & & & F F & $R$ & & $R$ & & & & & & & & & D & D & & & & & $\mathrm{R}$ & ? & & & 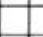 & 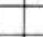 & & $c$ \\
\hline $5-4, \quad 117-118$ & 35.28 & & $R$ & $F$ & $R$ & 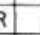 & & $\mathrm{F} / \mathrm{F}$ & $\begin{array}{ll}R & R \\
\end{array}$ & & $F$ & $R$ & & & & & & & & 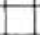 & t & & & & . & & $\mathrm{R}$ & & & & $\mathrm{R}$ & & C \\
\hline $5-6 . \quad 136-137$ & 38.47 & & $R$ & $\mathrm{~F}$ & & $R$ & & \begin{tabular}{l|l}
$F$ & $F$
\end{tabular} & \begin{tabular}{l|l}
$R$ & $R$ \\
\end{tabular} & q & $R$ & $R$ & & & & & & & & 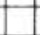 & $R$ & & & & F & \begin{tabular}{|l|l}
$R$ & $R$ \\
\end{tabular} & & & & 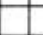 & 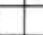 & & C \\
\hline $6-2,42-43$ & 41.03 & & & $F$ & R & | & & \begin{tabular}{|l|l|l}
$F$ & $F$
\end{tabular} & \begin{tabular}{l|l} 
R \\
\end{tabular} & & $R$ & & & & & & & & & Ot & $\mathrm{R}$ & & & & $F$ & a & 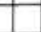 & & & & $\begin{array}{l}\mathbf{R} \\
\end{array}$ & & c \\
\hline 6-4. $42-43$ & 44.03 & & & $F$ & & & & $\mathrm{~F} F$ & $\begin{array}{ll}R & R \\
R\end{array}$ & & & & & & & & & & & $R$ & $R$ & & & & R & & $R$ & $R$ & & 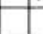 & 1 & 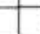 & A \\
\hline $6-6, \quad 117-118$ & 47.78 & & & $F$ & R & 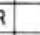 & & F F & \begin{tabular}{l|l} 
\\
$R$
\end{tabular} & & $R$ & $R$ & & & & & & & & & & & & & & R & & & & & R & & C \\
\hline $7-2, \quad 42-43$ & 50. 33 & & & $R$ & & & & $\begin{array}{lll}R & F \\
\end{array}$ & $R$ & & $R$ & & & & & & & & & 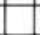 & $R$ & & & & 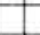 & & & & & 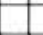 & 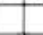 & & $F$ \\
\hline $7-4, \quad 42-43$ & 53.33 & & & $R$ & & & & $\mathrm{R}$ & & & & & & & & & & & & & & & & & & & & & & & 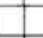 & & $F$ \\
\hline $7-6, \quad 42-43$ & 56.33 & & & $R$ & & & & & & & & & & & & & & & & & 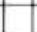 & & & & & & & & & & 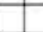 & & $F$ \\
\hline $8-1,42-43$ & 58.13 & & & $R$ & & & & RF & $R$ & & 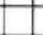 & & & & & & & & & & 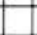 & & & & 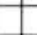 & & $\mathrm{R}$ & & & & 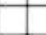 & 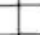 & c \\
\hline $8-2, \quad 42-43$ & 59.63 & & & $\mathrm{R}$ & I & & & $R$ & & & $R$ & 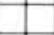 & & & & & & & & 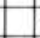 & $R$ & & & & & & & & & & $\mathrm{~F}$ & $\mathrm{R}$ & C \\
\hline $9-1,41-42$ & 67.62 & & & $\mathrm{R}$ & & & & $R$ & & & $R$ & & & & & & & & & R & & & & & R & & $R$ & & & & T & & $A$ \\
\hline $9-2 . \quad 7-8$ & 68.79 & & & $F$ & $R$ & & & F $F$ & R & & $R$ & 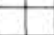 & & & & & $R$ & & & & Ot & & & & R & & & & & & $R$ & & C \\
\hline 9. $\mathrm{Cc}$ & 71.45 & $R$ & & $F$ & $R$ & q & & \begin{tabular}{l|l}
$C$ & $F$
\end{tabular} & R & & $R$ & & & $R$ & & & & & & 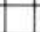 & $R$ & & & & R $F$ & g) & $R$ & & & \begin{tabular}{l|l}
$R$ & \\
\end{tabular} & \begin{tabular}{l|l}
$R$ & $R$ \\
\end{tabular} & R & c \\
\hline 10-2. $\quad 119-120$ & 79.40 & & & & & & & & & & & & & & & & & & & & & & & & & & & & & & & & $F$ \\
\hline 10-5. $\quad 119-120$ & 83,90 & & & $R$ & 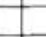 & & & $R$ & $R$ & & $R$ & & & & & & & & & 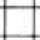 & & & & & & & & $R$ & & & & & $F$ \\
\hline 10-6. $\quad 119-120$ & 85.40 & & & $R$ & $R$ & & & $R$ & & & $R$ & $R$ & & & & & & & & $R$ & & & & & & & & & & & & & $F$ \\
\hline $11-2, \quad 98-99$ & 8840 & & & $R$ & & & & $R$ & & & & & & & & & & & & & & & & & & & $R$ & & & & 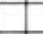 & & c \\
\hline $11-4,88-89$ & 91.59 & & & $R$ & & & & $R$ & & & R & & & & & & & & & & & & & & & & & & & & 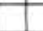 & & A \\
\hline $11-5.40-41$ & 92.61 & & & $F$ & & & & $F$ & $R$ & & $F$ & 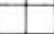 & & $\mathrm{R}$ & $R$ & & & & & & $R$ & & & & & & & $R$ & & T & $\mathrm{R}$ & & C \\
\hline $12-2 . \quad 39-40$ & 97.60 & & & $R$ & & & & $R$ & & & $R$ & 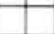 & & & & & & & & & & & & & & & & $R$ & & $R$ & 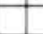 & & c \\
\hline $12-3, \quad 39-40$ & 99.10 & & & & & & & $R$ & & & $R$ & $R$ & & & & & & & & & & & & & & & & & & & $R$ & & $F$ \\
\hline $12-5, \quad 39-40$ & 102.10 & & & F F & R & & & & & & $R$ & & & & & & & & & 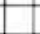 & $\square$ & & & & & & 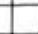 & & & & $R$ & & c \\
\hline $13-1.38-39$ & 105.59 & & & $R$ & & & & $R F$ & $\mathrm{R}$ & & $R$ & & & & & & & & & & $\mathrm{R}$ & & & & & & 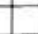 & & & & $\mathrm{R}$ & R & $F$ \\
\hline $14 . \mathrm{CC}$ & 114.70 & & & $F$ & & $R$ & & \begin{tabular}{l|l}
$R$ & $F$ \\
\end{tabular} & \begin{tabular}{l|l}
$R$ & $R$
\end{tabular} & & $F$ & & & & & & & & & & & & & & & & $R$ & & & & & & C \\
\hline $15 . \mathrm{CC}$ & 124.30 & & & $R$ & & & & & & & 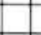 & & & & & & & & & & & & & & & & & & & $\mathrm{R}$ & & & $\mathrm{v}$ \\
\hline $16-1.42-43$ & 134.13 & $R$ & & $\mathrm{R}$ & & & & R & & & $R$ & & & & & & & & & & & & & & & & & & & & 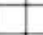 & & $F$ \\
\hline $16-2 . \quad 51-52$ & $\begin{array}{lll}135 & 72\end{array}$ & & & $R$ & & & & R F F & $R$ & & $R$ & 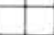 & & & & & & & & & $R$ & & & & & & & & & 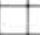 & & & $A$ \\
\hline $17 . \mathrm{Cc}$ & 143.40 & & & $R$ & & & & R & & & $R$ & & & & & & & & & & $R$ & & & & & & & & & & $R$ & & $A$ \\
\hline $18-1,32-33$ & 153.03 & & & $F$ & $R$ & & & F F & $\mathrm{R}$ & & R & $\mathrm{R}$ & & $R$ & & & & & & & & & & & & 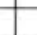 & $R$ & & & & R & & $\mathrm{F}$ \\
\hline $18-1, \quad 106-107$ & 153.77 & & & $F$ & $R$ & $R$ & & R & & & $R$ & & & $\mathrm{R}$ & & & & & & & & & & & & & & & & & & & C \\
\hline $19-1 . \quad 19-20$ & 162.37 & & & $R$ & & & & R & & & $\mathrm{R}$ & & & & & & & & & $R$ & & & & & & & & & & & $R$ & $R$ & c \\
\hline $19-2, \quad 10-11$ & 163.81 & & & $\mathrm{R}$ & & & & RF & \begin{tabular}{l|l}
$R$ & $R$ \\
\end{tabular} & & $R$ & & & & & & & & & & & & & & & & & & & & & & c \\
\hline $20-1,41-42$ & 172.12 & & & $R$ & & & & R & & & $R$ & & & & & & & & & & & & & & & $\mathrm{R}$ & & & & & $R$ & & c \\
\hline $20.0 \mathrm{c}$ & 173.25 & & & $R$ & & & & $R$ & & & $R$ & & & & & & & & & & $R$ & & & & & & $R$ & & & & R & & $\mathrm{F}$ \\
\hline $21-1,15-16$ & 181.36 & & & $R$ & $R$ & ? & & $R$ & & & $R$ & & & & & & & & & & & & & & & & & & & RF & \begin{tabular}{l|l}
$R$ & $R$ \\
\end{tabular} & 8 & c \\
\hline $21-1.91-92$ & 182.12 & & & $R$ & & & & R & & & 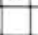 & & & & & & & & & & & & & & & & & & & & $\square$ & & A \\
\hline $22-1, \quad 46-47$ & 191.17 & & & $F$ & & & & RF & $\mathrm{R}$ & & $\mathrm{R}$ & $R$ & & & & & & & & & & & & & & & & & & & $R$ & & $A$ \\
\hline $22-3 . \quad 46-47$ & 194.17 & & & $F$ & & & & RF & R & & $R$ & & & & & & & & & & & & & & & & $R$ & & & & $R$ & & $A$ \\
\hline $22-5, \quad 38-39$ & 19709 & & & $F$ & R. & $R$ & & $\mathrm{FF}$ & $\mathrm{R}$ & & $\mathrm{A}$ & $R$ & & & & & & & & $R$ & $\mathrm{R}$ & & & & & & & & & & $\mathrm{R}$ & & c \\
\hline $22-6 . \quad 46-47$ & 19867 & & & $R$ & & & & R F F & $\begin{array}{ll}R & R \\
\end{array}$ & & & $R$ & & & & & & & & & $R$ & & & & & & & & & $R$ & & & c \\
\hline $22 . \mathrm{CC}$ & 199. 99 & & & $R$ & & & & R & & & $R$ & & & & & & & & & & & & & & & & & R & & & & $R$ & $A$ \\
\hline $23-1.95-96$ & 201.16 & & & $F$ & R & & & $\mathrm{FF}$ & \begin{tabular}{l|l}
$R$ & $R$ \\
\end{tabular} & & $R$ & & & & & & & & & & & & & & & & & & & & & & $A$ \\
\hline $23-2 . \quad 143-144$ & 203.14 & & & $R$ & & & & $\mathrm{~F}$ & $R$ & & $R$ & & & & & & & & & & & & & & & & & & & & & & $A$ \\
\hline $24 . \mathrm{CC}$ & 20972 & & & $F$ & $R$ & s & & $\mathrm{FF}$ & \begin{tabular}{l|l}
$R$ & $R$ \\
\end{tabular} & & $R$ & $R$ & & & & & & & & & & & & & & & & & & & $R$ & & $A$ \\
\hline $25 . \mathrm{cc}$ & 21930 & & & $R$ & & & & $\mathrm{FPF}$ & $\mathrm{R}$ & & $R$ & & & & & & & & & & $\mathrm{R}$ & & & & & & & & & $R$ & & & c \\
\hline $26 . \mathrm{cc}$ & 228.72 & $R$ & $R$ & $\mathrm{~F}$ & & $\mathrm{R}$ & & $\mathrm{F} F \mathrm{~F}$ & $\begin{array}{l}R \\
R\end{array}$ & & $F$ & & & & & & & & & & & & & & & R & & & & & & $R$ & C \\
\hline $27-1,20-21$ & 238.41 & & & $R$ & & $R$ & & R & & & $R$ & & & & & & & & & & & & & & & & & & & & & & $A$ \\
\hline
\end{tabular}


Table 7 (continued).

\begin{tabular}{|c|c|c|c|c|c|c|c|c|c|c|c|c|c|c|c|c|c|c|c|c|c|c|c|c|c|c|c|c|c|c|c|c|c|c|c|c|c|c|}
\hline $\begin{array}{l}\text { Core- } \\
\text { Section } \\
\text { (interval } \\
\text { in } \mathrm{cm} \text { ) }\end{array}$ & $\begin{array}{c}\text { Sub- } \\
\text { bottom } \\
\text { depth } \\
\text { (m) }\end{array}$ & & & & & & & & & & & & & & & & & & & & & & & & & & & & & & & & & & & 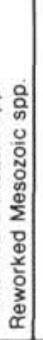 & 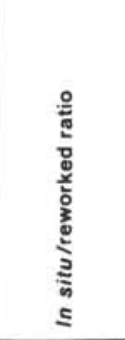 & $\begin{array}{l}\text { Zone or } \\
\text { subzone }\end{array}$ \\
\hline $27-2,42-43$ & 240.13 & & & & & & & & & & & & & & & & & & & & & & & & & & & & & & & & & & & $F$ & 0 & \multirow{27}{*}{$\begin{array}{c}\text { Emiliania } \\
\text { huxieyi } \\
\text { acme }\end{array}$} \\
\hline $28-1, \quad 22-23$ & 247.93 & & & F & & & & & $\mathrm{R}$ & & & $R$ & & & & & & & & & & & & & & & & & & & & & & & & c & os & \\
\hline $29-1, \quad 39-40$ & 25760 & & & R & & & & & $R$ & & & $R$ & $R$ & & & & & & & & & & & & & & & & & & & & & & & $A$ & 01 & \\
\hline $29-3 . \quad 39-40$ & 260.60 & & & F & & & & & R & & & $R$ & & & & & & & & & & & & & & & & & & & & & & & & C & .05 & \\
\hline $29-5 . \quad 39-40$ & 263.60 & & & F & & & & & R & & & $\mathrm{R}$ & & & & & & & & & & & & & & & & & & & & & & & & $F$ & 01 & \\
\hline $30-1, \quad 18-19$ & 266.89 & & & $\mathrm{~F}$ & & & & & $\begin{array}{ll}R \\
R\end{array}$ & $\mathrm{R}$ & & $R$ & $R$ & & & & & & & & & & & & & & & & & $\mathrm{R}$ & & & & $R$ & & $A$ & 01 & \\
\hline $32-1.41-42$ & 286.12 & & & F & & & & & R F & R & & $R$ & & & & & & & & & & & $\mathrm{R}$ & & & & & & & & $R$ & & & & & c & 1 & \\
\hline $32-2, \quad 7-8$ & 287.28 & & $\mathrm{R}$ & $R$ R & & $R$ & & & $R F$ & $\begin{array}{l}\mathrm{R} \\
\mathrm{R}\end{array}$ & & $R$ & $R$ & & & & & & $R$ & & & \begin{tabular}{l|l}
$R$ & $R$ \\
\end{tabular} & $\begin{array}{ll}\mathrm{R} & \mathrm{R} \\
\end{array}$ & & & & & $R$ & & $\mathrm{R}$ & & & & $R$ & & $A$ & $=1$ & \\
\hline $33-2.9-10$ & 306.30 & & & $\mathrm{~F}$ & & $R$ & & & $\begin{array}{l}R \\
R\end{array}$ & k| & & $R$ & $R$ & & & & & & & & & & $R$ & & & & & & & $\mathrm{R}$ & & & & & & c & .1 & \\
\hline $34-1,44-45$ & 314.65 & & & F & & & & & R & $\mathrm{A}$ & & $\mathrm{R}$ & & & & & & & & & & & $\mathrm{R}$ & & & & & & & & & & & & & $A$ & . os & \\
\hline $34-3.49-50$ & 317.70 & & & F & $R$ & & & & R F & 8 & & $R$ & & & & & & & & & & & & & & & & & & $\mathrm{R}$ & $R$ & & & & & $A$ & os & \\
\hline $35 . \mathrm{cc}$ & $323 \quad 80$ & & & F & & & & & R & & & $R$ & & & & & & & & & & & & & & & & & & & & & & & & $A$ & 01 & \\
\hline $36-1,46-47$ & 333.67 & & & R & $R$ & $R$ & & & R & & & $R$ & & & & & & & & & & & & & & & & & & & & & $R$ & & & $A$ & .01 & \\
\hline $38-1 . \quad 59-60$ & 352.80 & & & R & & & & & $R$ & & & $R$ & & & & & & & & & & & & & & & & & & & & & & & & $A$ & .01 & \\
\hline $38-2, \quad 53-54$ & 354.24 & & & F & & $\mathrm{R}$ & & & RF & & & & & & & & & & & & & & & & & & & & & & & & & & & c & 1 & \\
\hline $40-9.82-83$ & 372.03 & & & F & & $R$ & & & R F & $R$ & & $R$ & $\mathrm{R}$ & & & & & & & & & & & & & & & & & & & & & & & C & 1 & \\
\hline $42-1,3-4$ & 399.72 & & & $F$ & & $R$ & $R$ & & c $F_{F}$ & $R$ & & $F$ & $R$ & & $R$ & & & & & & & & \begin{tabular}{l|l}
$R$ & $R$ \\
\end{tabular} & & & & $R$ & & & $R$ & & & & $R$ & & $F$ & 5. & \\
\hline $43-1 . \quad 6-7$ & 409.27 & & & F & & & & & R & & & $R$ & & & & & & & & & & & & & & & & & & & & & & & & $F$ & . o5 & \\
\hline $43-4,70-71$ & 414.41 & & & F & & & & & & & & $R$ & & & & & & & & & & & & & & & & & & & & & & & & $F$ & .05 & \\
\hline $43-6, \quad 75-76$ & 417.46 & & & F & $R$ & & & & $\mathrm{R}$ & & & $R$ & & & & & & & & & & & & & & & & $R$ & & & & & & & & $F$ & 1 & \\
\hline $44-1,8-9$ & 418.79 & & & F & & & & & $\mathrm{R}$ & I & & $R$ & & & & & & & & & & & $\mathrm{R}$ & & & & & & & & & & & & & C & 1 & \\
\hline $44 . c c$ & 41900 & & & F & & & & & $\mathrm{R}$ & $\mathrm{R}$ & & $R$ & & & & & & & & & & & & & & & & & & $R$ & & & & & & C & .05 & \\
\hline $45 . \mathrm{CC}$ & 420.72 & & & & & & & & & & & & & & & & & & & & & & & & & & & & & & & & & & & $R$ & 0 & \\
\hline $46 . \mathrm{CC}$ & 438.05 & & & $F$ & & & & & RF & $R$ & & $R$ & & & $R$ & & & & & & & & R & R & & & & & & & & & & $R$ & & $F$ & 2 & \\
\hline $47-1.86-88$ & $457 \quad 57$ & & & F & & & & & R & & & $R$ & & & 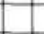 & & & & & & & & R & R & & & & & & 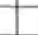 & & $R$ & & & & $F$ & 15 & \\
\hline $47-2 . \quad 9-10$ & 458.30 & & & F & & & & & R & & & $R$ & & & 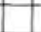 & & & & & & & & $R$ & & & & & & & & $R$ & & & & & C & , & \\
\hline $47 . \mathrm{CC}$ & 466.20 & & & $\mathrm{TF}_{\mathrm{F}}$ & $R$ & $R$ & $\mathrm{R}$ & & c $\mathrm{F}_{\mathrm{P}}$ & $R$ & & $\mathrm{~F}$ & $F$ & & 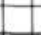 & & & & & & & & R $R$ & & & & $R$ & & F & $\mathrm{R}$ & $R$ & $R$ & $R$ & $R$ & & $F$ & 10. & \\
\hline $48, \mathrm{CC}$ & 485.20 & & $\begin{array}{r}R \\
R\end{array}$ & $\begin{array}{l}R A \\
A\end{array}$ & c & $F$ & c & R & $A$ & $\overline{C C}$ & $R$ & $\mathrm{v}$ & $\mathrm{R} C \mathrm{C}$ & & $F$ & R & $\begin{array}{l}R \\
\end{array}$ & & $R$ & $R$ & $\mathrm{~A}$ & $\mathrm{R} C \mathrm{C}$ & \begin{tabular}{l|l}
$C$ & $F$ \\
\end{tabular} & & $\mathrm{R}$ & $\mathrm{R}$ & $R$ & F & RF & $R$ & $R$ & $F$ & $R$ & $R F$ & & $A$ & 3.600 & \multirow{23}{*}{$\begin{array}{c}\text { Emiliania } \\
\text { huxleyi }\end{array}$} \\
\hline $49-1.42-43$ & 485,63 & & F & $R \mid A$ & c & $F$ & c & R & $A$ & CF & & $\mathrm{v}$ & C & & $F$ & & $F$ & q & & $R$ & R & $R \mid C$ & \begin{tabular}{l|l} 
c & $F$ \\
\end{tabular} & & & & $F$ & $F$ & $\mathrm{FF}$ & $=R$ & $R$ & $R$ & $\mathrm{R}$ & $\mathrm{R} F$ & & $\mathrm{R}$ & 5.400 & \\
\hline $49-2, \quad 42-43$ & 487,13 & & R. & $T_{A}$ & C & $F$ & c & R. & 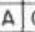 & $C F$ & & v & c & & $F$ & & & & & $R$ & $R$ & \begin{tabular}{l|l}
$R$ & $F$ \\
\end{tabular} & \begin{tabular}{l|l}
$F$ & $F$ \\
\end{tabular} & $F$ F & & $R$ & $F$ & $F$ & R F F & $=R$ & $\mathrm{R}$ & $R$ & $R$ & $\mathrm{R} F$ & C & & 2.900 & \\
\hline $49-3.42-43$ & 488.63 & & $R$ & v & C & $F$ & c & R & A & CF & R & $\mathrm{v}$ & c & & $F$ & & F & $\hat{R} \mathrm{R}$ & & $R$ & R & \begin{tabular}{l|l}
$\mathrm{R}$ & $\mathrm{F}$ \\
\end{tabular} & $\mathrm{F} F$ & $\mathrm{~F} / \mathrm{R}$ & & $R$ & $R$ & $\mathrm{~F}$ & R F & $=R$ & $R$ & $R$ & $R$ & R F & c & & 3.600 . & \\
\hline $49-4, \quad 42-43$ & 490.13 & & $R$ & $t_{v}$ & C & $F$ & c & R & A & \begin{tabular}{c|c}
$c$ & $F$ \\
\end{tabular} & & v & $R \mid C$ & & $F$ & & & & & $R$ & R & $\begin{array}{lll}R & F \\
\end{array}$ & $F$ F & $F R$ & & $R$ & $R$ & $F$ & RF F & $\begin{array}{l}F \\
F\end{array}$ & $R$ & $R$ & $R$ & $R / F$ & & $R$ & 6.000 & \\
\hline $49-5, \quad 42-43$ & 491.63 & & F & $R \mid A$ & C & $F$ & $\mathrm{~F}$ & R. & A & \begin{tabular}{c|c}
$c$ & $F$ \\
\end{tabular} & & $|A|$ & $\mathrm{c}$ & & $F$ & & & & & & R & \begin{tabular}{l|l}
$R$ & $F$ \\
\end{tabular} & $F$ F & & & & $R$ & $F$ & RFF & $=R$ & $R$ & $R$ & & R $P$ & & $A$ & 3. 000 & \\
\hline $49-6 . \quad 42-43$ & 493.13 & \begin{tabular}{|l|ll}
$R$ & \\
\end{tabular} & R & $T_{A}$ & C & $F$ & F & R. & $A$ & \begin{tabular}{c|c}
$C$ & $F$ \\
\end{tabular} & $R$ & $A$ & c & & $F$ & & & & & $R$ & & R F F & \begin{tabular}{l|l}
$R$ & $R$ \\
\end{tabular} & $R$ & & 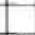 & $R$ & $R$ & RF F & $\begin{array}{l}\text { Q } \\
R\end{array}$ & $R$ & $R$ & & $R / F$ & & $R$ & 4. 000 & \\
\hline $50-1,42-43$ & 495.13 & & R & A & c & $F$ & c & & A F F & $F$ & & A & c & & $F$ & & & R & & & $R$ & \begin{tabular}{l|l}
$R$ & $F$ \\
\end{tabular} & $\mathrm{~F} O \mathrm{C}$ & c & & $R$ & $F$ & $F$ & R F F & $\mathrm{R}$ & $F$ & $R$ & $R$ & tF & & $R$ & 3.600 . & \\
\hline $50-2 . \quad 42-43$ & 496.63 & & R F & $R A$ & C & $F$ & c & R. & A & \begin{tabular}{c|c}
$C F$ \\
C
\end{tabular} & & v & $\mathrm{F}$ & & $F$ & & F & a. & & $\mathrm{R}$ & $R$ & $F$ & $\mathrm{~F} C \mathrm{C}$ & c & & $\mathrm{R}$ & $F$ & $R$ & R F & $\begin{array}{l}\text { R } \\
F\end{array}$ & R & $R$ & & $\mathrm{R}$ & $c \mid c$ & $R$ & 5.400. & \\
\hline $50-3.42-43$ & 498.13 & & & $\begin{array}{ll}R & A \\
\end{array}$ & C & $F$ & c & R & $A$ & \begin{tabular}{c|c}
$c$ & $F$ \\
\end{tabular} & & $\mathrm{v}$ & $\begin{array}{ll}\mathrm{R} & \mathrm{C} \\
\end{array}$ & & $F$ & & & R & & & R & \begin{tabular}{l|l}
$R$ & $F$ \\
\end{tabular} & $F \mid F$ & $\begin{array}{ll}F & R \\
\end{array}$ & $R$ & $\mathrm{R}$ & $F$ & R & RF F & $\begin{array}{l}\mathrm{R} \\
\mathrm{F}\end{array}$ & $F$ & $R$ & $R$ & \begin{tabular}{l|l}
$R$ & \\
\end{tabular} & A & & 4,800 & \\
\hline $50-4,42-43$ & 499.63 & & R & $T_{A}$ & C & $F$ & c & R & A & \begin{tabular}{c|c}
$c$ & $F$ \\
\end{tabular} & $R$ & $\mathrm{v}$ & $\mathrm{R} C \mathrm{C}$ & & $F$ & & $F$ & Q. & $R$ & & $R$ & \begin{tabular}{l|l}
$R$ & $F$ \\
\end{tabular} & F F & $\mathrm{F}$ & $R$ & $R$ & $F$ & \begin{tabular}{l|l}
$R$ & 1 \\
\end{tabular} & RFF & $=F$ & $F$ & $F$ & $R$ & th & & $A$ & 6.000. & \\
\hline $50-5, \quad 42-43$ & 501.13 & & $\begin{array}{ll}R \\
\end{array}$ & $\begin{array}{l}R \\
R\end{array}$ & c & $F$ & c & R. & A & \begin{tabular}{c|c}
$c$ & $F$ \\
\end{tabular} & & $\mathrm{v}$ & c & & $F$ & & & & & $R$ & & $\begin{array}{lll}R & F \\
\end{array}$ & $F \mid F$ & & & $R$ & $R$ & $F$ & $R$ F & $=R$ & $R$ & $F$ & $R$ & RIf & & $A$ & 6.000 & \\
\hline $50-6 . \quad 42-43$ & 502.63 & $R$ & F & $R \mid A$ & C & $F$ & c & R & $A C$ & \begin{tabular}{c|c}
$c$ & $F$ \\
\end{tabular} & $R$ & $\mathrm{~V}$ & c & & $F$ & & & & $R$ & $R$ & $\mathrm{R}$ & \begin{tabular}{l|l}
$R$ & $F$ \\
\end{tabular} & $F \mid F$ & $\mathrm{~F} F$ & $R$ & $\mathrm{R}$ & $F$ & $F$ & R F F & $=R$ & $R$ & $R$ & & $\mathrm{R}$ & C & & 6.000 & \\
\hline $51-1,41-42$ & 504.62 & & & $T_{A}$ & ic & $F$ & c & $\mathrm{R}$. & $A \mid C$ & $c \mid F$ & $R$ & v & c & & $F$ & R & A & & $\mathrm{R}$ & & $R$ & \begin{tabular}{l|l}
$R$ & $F$ \\
\end{tabular} & $\mathrm{~F} F \mathrm{~F}$ & $\mathrm{~F}$ & & R & $F$ & F & R F F & $=F$ & $R$ & $A$ & & ti & C & & 6. 000 . & \\
\hline $51-2,41-42$ & 506.12 & & & R $A$ & C & $F$ & c & & A & \begin{tabular}{c|c|c}
$C$ & $F$ \\
\end{tabular} & & v & c & & $F$ & & & R & $R$ & & $R$ & \begin{tabular}{l|l}
$R$ & $F$ \\
\end{tabular} & $\mathrm{~F} F \mathrm{~F}$ & $\mathrm{R}$ & & R & $R$ & $F$ & RF F & $=F$ & $R$ & $R$ & $R$ & & & $\mathrm{~A}$ & 6.000 & \\
\hline $51-3,41-42$ & 50762 & & R $F$ & $R A$ & C & C & c & $R$ & A & c c & & $\mathrm{v}$ & c & & $F$ & & $F$ & Q & & $R$ & $R$ & R F & FF F & $F$ & $R$ & $R$ & $R$ & F & Ff & $=F$ & $\mathrm{~F}$ & $R$ & $R$ & $\mathrm{R}$ & c & $R$ & 6.000 & \\
\hline $51-4,41-42$ & 509.12 & & & $T_{A}$ & c & $F$ & c & & A & c & $R$ & $\mathrm{v}$ & $\mathrm{R} / \mathrm{C}$ & & $F$ & & & & $\mathrm{R}$ & $R$ & $R$ & \begin{tabular}{l|l}
$R$ & $F$ \\
\end{tabular} & $F F$ & \begin{tabular}{l|l}
$F$ & $R$ \\
\end{tabular} & $R$ & $R$ & $F$ & $F$ & $R F$ & $F$ & $R$ & $R$ & $R$ & ti & C & & 6.000 . & \\
\hline $51-6.41-42$ & 510.62 & & 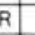 & TA & C & $F$ & c & R & A & \begin{tabular}{c|c}
$C$ & $F$ \\
\end{tabular} & & $\mathrm{v}$ & C & & $F$ & & & R & $R$ & $R$ & $R$ & $\begin{array}{lll}R & F \\
\end{array}$ & $F$ F & \begin{tabular}{l|l}
$F$ & $F$ \\
\end{tabular} & & $R$ & $F$ & $F$ & RF & $=R$ & $F$ & $R$ & & R & $c$ & & 6.000 & \\
\hline $51 . \mathrm{cc}$ & 513.20 & f & R $\mathrm{F}$ & RA & c & $F$ & c & R. & A & \begin{tabular}{c|c|c|}
$c$ & $F$
\end{tabular} & $R$ & v & c & & $F$ & & & $R$ & $R$ & & $\mathrm{R}$ & \begin{tabular}{l|l}
$R$ & $F$ \\
\end{tabular} & $F F$ & $\mathrm{~F}$ & $R$ & $R$ & $F$ & $F$ & $\mathrm{~F} F$ & $=7$ & $\mathrm{~F}$ & R & $R$ & R & C & & 10.000 & \\
\hline $52-1,40-41$ & 514.11 & $\mathrm{R}$ & F & $F$ & $R$ & $F$ & c & $\mathrm{R}$ & c. & $A C$ & & $\mathrm{v}$ & c & & $\mathrm{R}$ & & $F$ & a $\mathrm{R}$ & & & $R$ & R & c & c & & $\mathrm{R}$ & $F$ & $F$ & Ff & $=F$ & $F$ & $\mathrm{R}$ & $F$ & $R$ & $\mathbf{R}$ & & 10.000 & \\
\hline $52-1 . \quad 105-106$ & 514.76 & & $F$ & $F[A$ & $R$ & $F$ & c & $\mathrm{R}$ & c) & $A C$ & & v & c & & $R$ & $R$ & F & a & $R$ & $R$ & $R$ & R & c) & c & & $\mathrm{F}$ & $F$ & $F$ & Ff & $=F$ & $F$ & $R$ & $F$ & $\mathrm{R}$ & $R$ & $R$ & 9.000 & \\
\hline $52-1, \quad 117-118$ & 514.88 & $\mathrm{R}$ & $\mathrm{F}$ & R & $R$ & $F$ & c & R & c. & $A C$ & & $\mathrm{v}$ & c & & $F$ & & $F$ & a $\mathrm{R}$ & & & $R$ & R & c) & c & & $F$ & $F$ & $\begin{array}{ll} \\
\end{array}$ & Ff & $=F$ & $F$ & $R$ & $F$ & $F$ & $\mathrm{~F}$ & $\mathrm{R}$ & 10.000 & \\
\hline $52 . \mathrm{CC}$ & $515 \quad 24$ & & & $\mathrm{~F}$ & & & & & R & $\mathrm{R}$ & & $\mathrm{F}$ & R & & & & & & & & & & & & & & & & & & R & & & & Q & c & 5 & \\
\hline
\end{tabular}

Note: For abundance, $V=$ very abundant, $10-100$ specimens $/$ field; $A=$ abundant, $1-10$ specimens $/$ field: $C=$ common, 1 specimen $/ 2-10$ fields: $F=$ few, 1 specimen/11-100 fields; $R=$ rare, 1 specimen/101-1000 fields (when viewed at a magnification of $\times 1250$ ). The depth of Sample, $48, C C$ has been adjusted to the bottom of the cored interval, as the shipboard scientists think that it came from that depth (Site 615 chapter, this volume): standard DSDP convention would place it at $475.7 \mathrm{~m}$ (Explanatory Notes, this volume).

ficult. Therefore, all samples are placed in the E. huxleyi Zone of Boudreaux and Hay.

A slight increase in Pleistocene species is found in Cores 616-9 through 616-10. This increase corresponds to an increase in planktonic and benthic foraminifers and may represent a period of slower sedimentation.

\section{Intraslope Basin Sites}

Salt diapirism on the Louisiana continental slope is responsible for the formation of a hummocky bottom topography. The depressions between the salt diapirs are often filled with thick sequences of sediments. Two types 
R. E. CONSTANS, M. E. PARKER

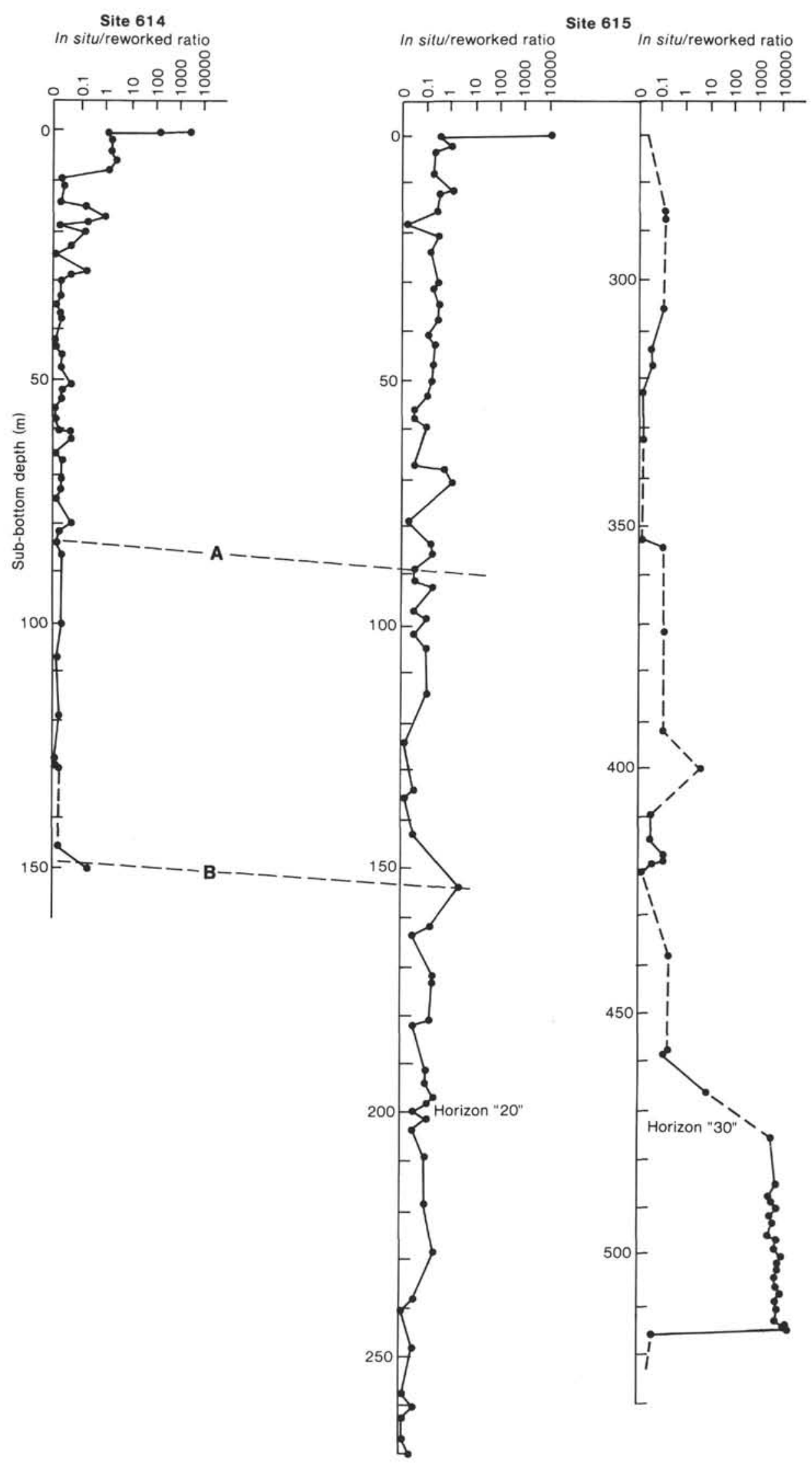

Figure 4. Correlation of the in situ/reworked ratio curves for Sites 614 and 615. Lines A and B indicate probable correlations between sites. The placements of regional seismic Horizons " 20 " and " 30 " (introductory chapter, this volume) are shown for Site 615. 
Table 8. Distribution of calcareous nannofossils, Site 614.

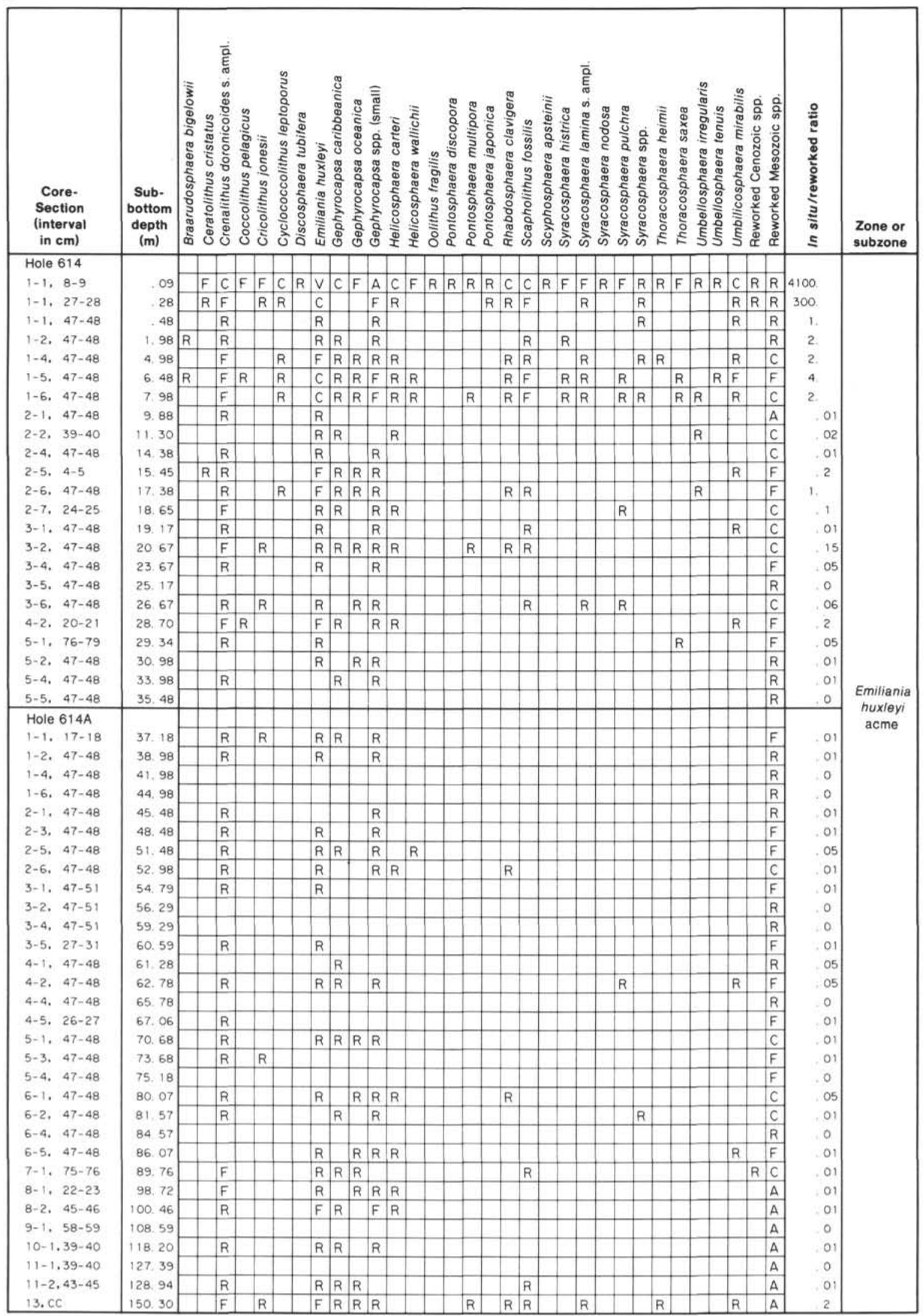

Note: For abundance, $\mathrm{V}=$ very abundant, $10-100$ specimens/field; $\mathrm{A}=$ abundant, $1-10$ specimens/field: $\mathrm{C}=\mathrm{common}, 1$ specimen $/ 2-10$ fields; $F=$ few, 1 specimen/11 - 100 fields: $R=$ rare, 1 specimen $/ 101-1000$ fields (when viewed at a magnification of $\times 1250$ ). 
Table 9. Distribution of calcareous nannofossils, Site 616.

\begin{tabular}{|c|c|c|c|c|c|c|c|c|c|c|c|c|c|c|c|c|c|c|c|c|c|c|c|c|c|c|c|c|}
\hline $\begin{array}{l}\text { Core- } \\
\text { Section } \\
\text { (interval } \\
\text { in cm) }\end{array}$ & $\begin{array}{c}\text { Sub- } \\
\text { bottom } \\
\text { depth } \\
\text { (m) }\end{array}$ & 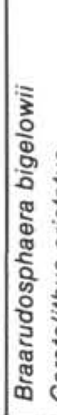 & 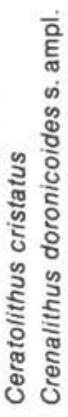 & 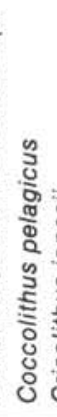 & & 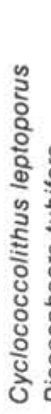 & & 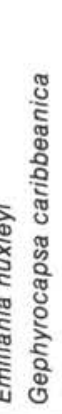 & 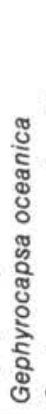 & & 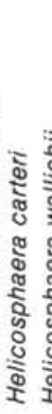 & & 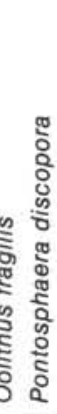 & 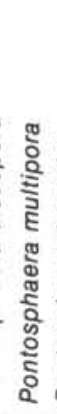 & 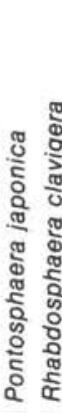 & 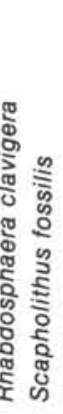 & & 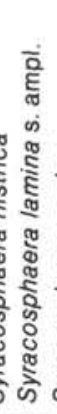 & 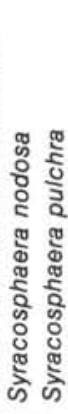 & 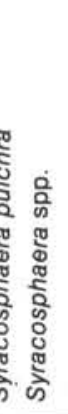 & 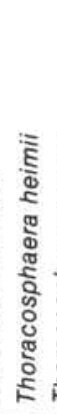 & 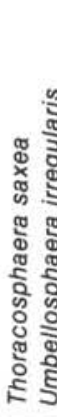 & 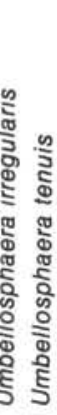 & 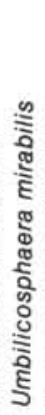 & 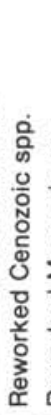 & 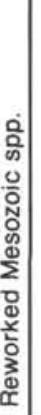 & 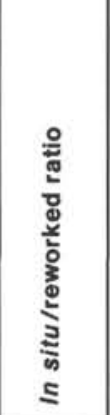 & $\begin{array}{l}\text { Zone or } \\
\text { subzone }\end{array}$ \\
\hline $1-1,0-2$ & .02 & & \begin{tabular}{l|l}
$R$ & $C$ \\
\end{tabular} & $R$ & $R$ & $\mathrm{~F} F$ & \begin{tabular}{l|l}
$R$ & $A$ \\
\end{tabular} & $\begin{array}{ll}A & R \\
\end{array}$ & & $R$ & $\mathrm{~F}$ & $\mathrm{~F} F$ & R & $\mathrm{R}$ & $R$ & $\begin{array}{ll}2 \\
\end{array}$ & $R$ & $R$ & \begin{tabular}{l|l}
$R$ & $R$ \\
\end{tabular} & $R$ & $\mathrm{R} / \mathrm{F}$ & \begin{tabular}{l|l}
$F$ & $R$ \\
\end{tabular} & \begin{tabular}{l|l}
$R$ & $R$ \\
$R$
\end{tabular} & $F$ & $f$ & $R^{2}$ & 500 & \multirow{48}{*}{$\begin{array}{c}\text { Emiliania } \\
\text { huxleyi }\end{array}$} \\
\hline $1-1.15-17$ & .17 & & & & & & & & & & & & & & & & & & & & & & & & & & Barren & \\
\hline $1-1,20-22$ & .22 & & & & & & & & & & & & & & & & & & & & & & & & & & Barren & \\
\hline $1-1.41-42$ & 42 & & \begin{tabular}{l|l}
$R$ & $F$ \\
\end{tabular} & & & $\mathrm{R}$ & $\mathrm{F}$ & $=R$ & $R$ & $\mathrm{R}$ & $R$ & $R$ & & & $R$ & $R$ & & & & $R$ & $R$ & & & $F$ & f & $\mathrm{F}$ & 1. & \\
\hline $1-2 \cdot 41-42$ & 1. 92 & & $F$ & & $R$ & $\mathrm{R}$ & $\mathrm{F}$ & $=R$ & $F$ & $R$ & $R /$ & $R$ & & & $R$ & $\mathrm{R} / \mathrm{F}$ & & & & & 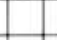 & & R & $R$ & & $F$ & 2. & \\
\hline 1-3. $41-42$ & 3. 42 & & $F$ & & $\mathrm{R}$ & $R$ & $\mathrm{~F}$ & $=R$ & $R$ & $R$ & $R /$ & $R$ & & & $R$ & $\mathrm{R} R$ & $R$ & & & & $\mathrm{R}$ & \begin{tabular}{l|l}
$R$ & $R$ \\
\end{tabular} & \begin{tabular}{l|l}
$\mathrm{R}$ & \\
\end{tabular} & $F$ & & $F$ & 1. & \\
\hline 1-4. $41-42$ & 4. 92 & & $R$ & & & & $R$ & $R$ & & $R$ & $R$ & & & & & $R$ & & & & & $R$ & & & $R$ & f & $\mathrm{F}$ & 2 & \\
\hline $2-1.42-43$ & 6. 53 & & $R$ & & & $R$ & $R$ & $R$ & & $R$ & & $R$ & & & $R$ & \begin{tabular}{l|l}
$R$ & $R$ \\
\end{tabular} & & & & & & & & $R$ & & $F$ & .05 & \\
\hline $2-3.42-43$ & 9. 53 & & $R$ & & $R$ & $\mathrm{R}$ & $\mathrm{F}$ & $=R$ & & $R$ & $R$ & & & & R & $R$ & & & & & & & & $R$ & f & $F$ & .6 & \\
\hline $2-5, \quad 42-43$ & 12. 53 & & $R$ & & & & $R$ & 2 & & $\mathrm{R}$ & & & & & & & & & & $R$ & & & & & & C & .05 & \\
\hline $3-1.42-43$ & 16.03 & & $R$ & & & & $R$ & R & & $R$ & $R$ & & & & & & & & & & & & & & & C & .01 & \\
\hline $3-2, \quad 42-43$ & 17.53 & & $R$ & & & & $R$ & \begin{tabular}{l|l|}
$R$ & $R$ \\
\end{tabular} & & $R$ & & & & & & & & & & & & & & & & C & .01 & \\
\hline $3-4,42-43$ & 20.53 & & $F$ & & & & $F$ & $=R$ & $R$ & $R$ & & & & & & $R$ & & & $R$ & & & & & 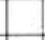 & & C & 1 & \\
\hline $3-6, \quad 42-43$ & 23.53 & & $R$ & & & & $\mathrm{~F}$ & $=R$ & & $\mathrm{R}$ & $R$ & & & & $R$ & $R$ & & & & & & & & $R$ & & $\mathrm{~F}$ & 1. & \\
\hline $4-1,42-43$ & 25.53 & & $\mathrm{~F}$ & & 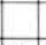 & 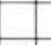 & C & $C R$ & $R$ & $\mathrm{R}$ & $R$ & & & & $R$ & $R$ & & & & & & & & $R$ & f & $\mathrm{F}$ & 1. & \\
\hline $4-3,27-28$ & 28. 38 & & $F$ & & $R$ & \begin{tabular}{l|l}
$R$ & $F$ \\
\end{tabular} & \begin{tabular}{l|l}
$R$ & $C$ \\
\end{tabular} & $C A$ & $R$ & & $R$ & & & & R & $R$ & & & & & & $R$ & \begin{tabular}{l|l}
$R$ & $R$ \\
\end{tabular} & $R$ & $R$ & $F$ & 5. & \\
\hline $4-5, \quad 42-43$ & 31.53 & & $F$ & & & & $F$ & $=R$ & & $R$ & $R /$ & $R$ & & $R$ & $R$ & $R$ & & $R$ & $R$ & R & & & & $R$ & P & $F$ & 3. & \\
\hline $4-6 . \quad 42-43$ & 33,03 & $R$ & F & & $R$ & $\mathrm{R}$ & C & c & & & $R F$ & $\mathrm{R}$ & & & $R$ & $R$ & & & R & 8 & & $R$ & R & $\mathrm{R}$ & $R$ & $F$ & 5. & \\
\hline $5-1, \quad 33-34$ & 34. 93 & & $\mathrm{~F}$ & & & $R$ & $\mathrm{~F}$ & $=R$ & $R$ & $R$ & $R$ & & & & & $R$ & & & & & & & & & & C & 1. & \\
\hline $5-3, \quad 33-34$ & 37.93 & & $R$ & & & & R & $R$ & & & & & & & $R$ & & & & & & & & & & & c & 1 & \\
\hline $5-5 . \quad 33-34$ & 40.93 & & $R$ & & & & $R$ & R & & $R$ & & & & & $R$ & & & & & & & $R$ & \begin{tabular}{l|l}
$R$ & $R$ \\
\end{tabular} & & & A & .01 & \\
\hline $5-6, \quad 33-34$ & 42. 44 & & $\mathrm{~F}$ & & & & $R$ & $R$ & & & $R$ & & & & & $R$ & & & & & & & $R$ & $R$ & & C & 01 & \\
\hline $6-1.69-70$ & 44. 90 & & $R$ & & & & $R$ & \begin{tabular}{ll|}
$R$ & $R$ \\
\end{tabular} & & $R$ & & & & & $R$ & $R$ & & & & & & & & $R$ & & A & 05 & \\
\hline 6-3. $69-70$ & 47.90 & & $R$ & & & & $\mathrm{~F}$ & $=1$ & $R$ & $R$ & & $R$ & & & & $R$ & & & & & & $R$ & $R$ & $R$ & & A & .05 & \\
\hline $6-5,69-70$ & 50.90 & & $R$ & & & & $R$ & R & $R$ & & & & & & & $R$ & & & & & & & & & & A & 01 & \\
\hline $6 . \mathrm{CC}$ & 52. 32 & & $R$ & & & & R & 2 & & $R$ & & & & & & $R$ & & & & & & & & 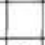 & & A & .01 & \\
\hline $7-1.65-66$ & 54.46 & & $F$ & & & & $R$ & $R$ & & & $R$ & $R$ & & & & $R$ & & & & & & & & $R$ & & A & .01 & \\
\hline $7-3,65-66$ & 57.46 & & & & & & $R$ & \begin{tabular}{l|l} 
\\
\end{tabular} & & $R$ & & & $R$ & & $R$ & $R$ & & & & & $R$ & & & & & A & .01 & \\
\hline $7-5,65-66$ & 60.46 & & $R$ & & $R$ & & $\mathrm{~F}$ & $R$ & $R$ & $R$ & & & & & $R$ & $R$ & & & & & $R$ & & & & & A & 01 & \\
\hline $8-1 \cdot 81-82$ & 64. 22 & & $R$ & $R$ & & & $R$ & $R$ & & $R$ & & & & & & $R$ & & $R$ & & $R$ & $R$ & 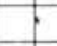 & $R$ & & & A & .01 & \\
\hline $8-3,81-82$ & 67.22 & & $R$ & & $\mathrm{R}$ & $R$ & $F$ & $=R$ & $R$ & & $R$ & & & & & & & & & & & & & & & C & .05 & \\
\hline $9-1,36-37$ & 73. 37 & & $R$ & & $R$ & & $R$ & 2. & & $R$ & & & & & $R$ & $R$ & & & & & & & $R$ & & & A & .01 & \\
\hline $9-3, \quad 36-37$ & 76. 37 & & $F$ & R & $R$ & & $R$ & $R$ & & $R$ & & & & & & & & & & & & & & & & A & .05 & \\
\hline 9. $\mathrm{CC}$ & $78: 20$ & & $\mathrm{~F}$ & $R$ & & $\mathrm{R}$ & C & \begin{tabular}{ll|}
$C$ & $R$ \\
\end{tabular} & $F$ & $R$ & $R$ & & & & $R$ & R F & $R$ & $R$ & $R$ & $R \mid R$ & & \begin{tabular}{l|l}
$R$ & $R$ \\
\end{tabular} & \begin{tabular}{l|l}
$\mathrm{R}$ & $\mathrm{R}$ \\
\end{tabular} & $R$ & & C & 5. & \\
\hline $10-1, \quad 47-48$ & 83. 07 & & $\mathrm{~F}$ & & & & $\mathrm{~F}$ & $=R$ & $R$ & & & & & & $R$ & $R$ & & & & & $\mathrm{R}$ & \begin{tabular}{l|l} 
& $R$ \\
\end{tabular} & \begin{tabular}{l|l}
$R$ & \\
\end{tabular} & $R$ & & C & 2. & \\
\hline $10-3, \quad 47-48$ & 86. 07 & & $R$ & & & & $R$ & $R \mid R$ & $R$ & $R$ & & & & & & $R$ & & & & & & & & & & C & .1 & \\
\hline $11-1,63-64$ & 92. 84 & $R$ & $F$ & & & & $R$ & \begin{tabular}{l|l}
2 \\
\end{tabular} & $R$ & $R$ & & & & & & $R$ & & & & & & & & $R$ & 8 & C & 05 & \\
\hline $11-3.63-64$ & 95.84 & & $R$ & $R$ & & $\mathrm{R}$ & $\mathrm{F}$ & $=R$ & & & & $\mathrm{R}$ & $R$ & & & & & & & & & & & & & A & .05 & \\
\hline $13-1.47-48$ & 114.07 & & $R$ & & & & $R$ & $\begin{array}{ll}2 \\
\end{array}$ & & $R$ & & $R$ & & & & & & & & & & & & $R$ & & C & .05 & \\
\hline $13 . \mathrm{CC}$ & 115.02 & & $F$ & & & & $R$ & $R$ & $R$ & $R$ & $R$ & & & & & & & & & & & & & & & C & .05 & \\
\hline $14 . \mathrm{CC}$ & 123. 22 & & $F$ & & & & $R$ & 2. & $R$ & $\mathrm{R}$ & $R$ & & & & & & & & & & & & & & $R$ & C & .05 & \\
\hline $15 . \mathrm{CC}$ & 132.82 & & $R$ & $R$ & & & $\mathrm{R}$ & R & $R$ & & & & & & & & & & & & & & & $R$ & $\mathrm{R}$ & $F$ & 075 & \\
\hline $16-1,81-82$ & 143.22 & & $R$ & & & & $R$ & \begin{tabular}{l|l} 
R \\
\end{tabular} & & $R$ & $R$ & & & & & & & & & & & & & & & C & .05 & \\
\hline $16-3,81-82$ & 146.22 & & $F$ & & & & $R$ & $R$ & & & & & & & & & & & & & & & & & & C & 01 & \\
\hline $16 . \mathrm{CC}$ & 149.02 & & $R$ & $R$ & & & $\mathrm{~F}$ & $=R$ & R & & & & & & R & & & & & & & & & $R$ & 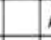 & A & .05 & \\
\hline $17-1,69-70$ & 152.70 & & $F$ & & $R$ & & $\mathrm{R}$ & $R$ & & $R$ & & & & & & & & & & & & & & & $R$ & c & .05 & \\
\hline $17-3, \quad 69-70$ & 155.70 & & $R$ & & & & $R$ & $R$ & $R$ & $R$ & & & & & & & & & & & & & & & & C & 01 & \\
\hline $18-1.98-99$ & 162.59 & & $R$ & & & & & $R$ & & $R$ & $R$ & & & & & & & & & & & & & & & C & 01 & \\
\hline
\end{tabular}


Table 9 (continued).

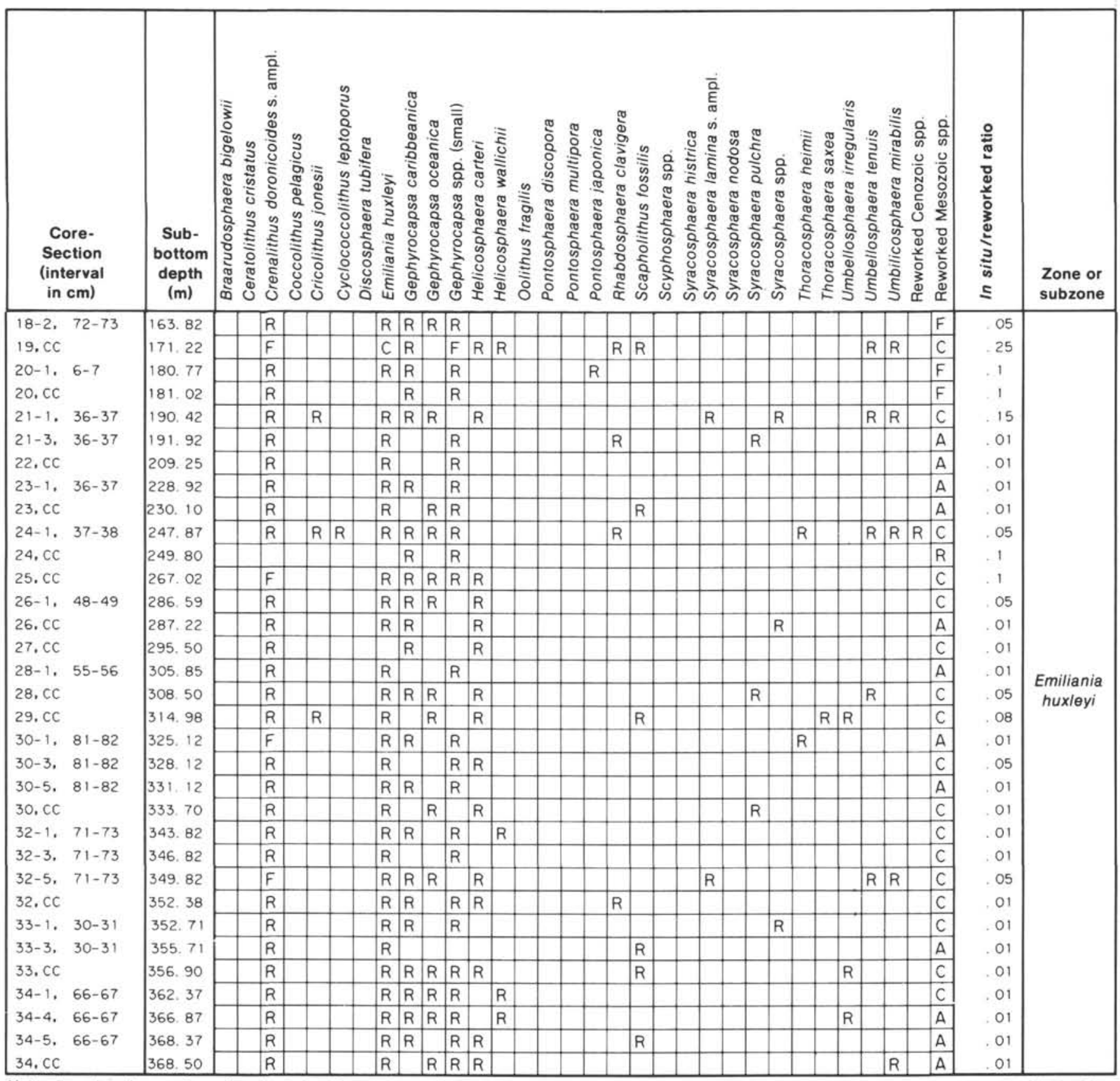

Note: For abundance, $A=$ abundant, $1-10$ specimens/field; $C=$ common, 1 specimen $/ 2-10$ fields; $F=$ few, 1 specimen $/ 11-100$ fields: $R=$ rare, 1 specimen/101-1000 fields (when viewed at a magnification of $\times 1250$ ).

of these intraslope basins were drilled during Leg 96 at Sites 618 and 619 (Intraslope Basin Introduction and Summary, this volume).

\section{Site 618}

Site $618\left(27^{\circ} 00.68^{\prime} \mathrm{N}, 91^{\circ} 15.73^{\prime} \mathrm{W}\right.$; water depth $2422 \mathrm{~m}$ ), located in the Orca Basin, was hydraulic piston cored to a depth of $92.5 \mathrm{~m}$ sub-bottom. The diapiric walls of this interdomal basin rise up to $700 \mathrm{~m}$ above the basin floor, which is covered by a 200 -m-thick layer of anoxic, hypersaline brine. Anoxic, dark-black clays alternate with gray clays to a depth of $41 \mathrm{~m}$ sub-bottom.
Reworked Pliocene nannofossils are common in most samples (Table 10). Discoaster pentaradiatus, D. brouweri, and Sphenolithus abies occur most often. Miocene and Eocene species show sporadic occurrences and include $D$. quinqueramus, $D$. bollii, and $D$. barbadiensis. The presence of Emiliania huxleyi in all samples, however, places all the sediments recovered at this site in the E. huxleyi Zone of Boudreaux and Hay.

Carbonate clasts averaging $0.5 \mathrm{~mm}$ in size are found in washed foraminifer Samples 618-4-4, 120-126 cm, 618-7,CC, and 618A-3,CC (Plate 4, Fig. 1). These clasts contain common to abundant, moderately preserved nan- 
Table 10. Distribution of calcareous nannofossils, Site 618 .

\begin{tabular}{|c|c|c|c|c|c|c|c|c|c|c|c|c|c|c|c|c|c|c|c|c|c|c|c|c|c|c|c|c|c|c|c|c|c|c|c|}
\hline $\begin{array}{l}\text { Core- } \\
\text { Section } \\
\text { (interval } \\
\text { in } \mathrm{cm} \text { ) }\end{array}$ & $\begin{array}{c}\text { Sub- } \\
\text { bottom } \\
\text { depth } \\
\text { (m) }\end{array}$ & 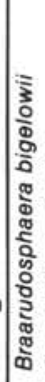 & & & & & & & & & & & & & & & & & & & & & & & & & & & & & & & 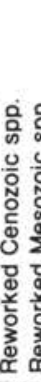 & 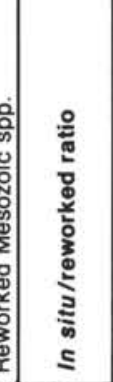 & $\begin{array}{l}\text { Zone or } \\
\text { subzone }\end{array}$ \\
\hline $1-1,0-1$ & .01 & & \begin{tabular}{|l|l}
$R$ \\
\end{tabular} & \begin{tabular}{c|c|}
${ }_{1}$ &
\end{tabular} & $\begin{array}{ll}R \\
R\end{array}$ & $\bar{F} F$ & $F F$ & $\mathrm{R} A$ & $\sqrt{A}$ & C & $A$ & F & $F$ & & $R$ & & & $\mathrm{R}$ & $\mathrm{F}$ & $R$ & & & & $\mathrm{R}$ & $\bar{F} F$ & 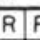 & $\bar{F} F$ & $\begin{array}{l}\mathrm{R} F \\
\mathrm{~F}\end{array}$ & R & $\mathrm{R}$ & $R$ & $F$ & $\mathrm{R}$ & 72. & \multirow{56}{*}{$\begin{array}{c}\text { Emiliania } \\
\text { huxleyi }\end{array}$} \\
\hline $1-2.120-121$ & 2.71 & $R$ & $\mathrm{R}$ & c & 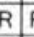 & $\mathrm{F} / \mathrm{F}$ & & A & $A$ & c & A & $\mathrm{F}$ & $\mathrm{F}$ & R & & & $F$ & & C & $F$ & & & $R$ & $R$ & $F$ & \begin{tabular}{l|l}
$R$ & $F$ \\
\end{tabular} & $\mathrm{~F} F$ & R $R$ & $R$ & & $R$ & $\mathrm{~F}$ & & 80. & \\
\hline $1-3, \quad 115-116$ & 4. 16 & $R$ & $R$ & c] & $R \mid F$ & $\mathrm{~F} / \mathrm{F}$ & $=\mathrm{F}$ & R A & $A$ & c & A & $\mathrm{F}$ & $\mathrm{F}$ & & & & & $\mathrm{R}$ & c & $F$ & & & & $R$ & $\mathrm{~F} F \mathrm{~F}$ & $\mathrm{~F} F$ & $F$ P & R $R$ & & & $R$ & $\mathrm{~F}$ & $\mathrm{~F}$ & 40. & \\
\hline $1-4, \quad 105-106$ & 5. 56 & & $R$ & c & RF & $\mathrm{F} / \mathrm{F}$ & & A & Ac & c & c & c) & $\mathrm{F}$ & & F & $R$ & & & C & $\mathrm{F}$ & & & & $R$ & $\mathrm{~F} F \mathrm{~F}$ & $F \mid F$ & & R & & $R$ & & c| & $\bar{F}$ & 35. & \\
\hline $1-5.40-41$ & 6. 41 & $R$ & $R$ & c & $R / F$ & $\mathrm{~F} / \mathrm{F}$ & $=\mathrm{F}$ & a. $v$ & 10 & $c$ & A & $\mathrm{F}$ & $F$ & & $F$ & $\mathrm{R}$ & & R & c & $R$ & & & \begin{tabular}{l|l}
$R$ \\
\end{tabular} & R & \begin{tabular}{c|c|c|}
$F$ \\
$F$
\end{tabular} & $F F$ & & $\mathrm{~F}$ & $\mathrm{R}$ & & $R$ & c & C & 167. & \\
\hline $1 . c c$ & 6. 50 & $\mathrm{R}$ & $R$ & c) & F F & $\mathrm{F} F$ & $=\mathrm{F}$ & a v & $1 \mathrm{c}$ & C & A & $\mathrm{F}$ & R & R & F & $R$ & F & $R$ & C & $F$ & & \begin{tabular}{|l|l|l|l}
$R$ \\
\end{tabular} & $\mathrm{R}$ & F & $\mathrm{R} F \mathrm{~F}$ & \begin{tabular}{l|l}
$\mathrm{R}$ & $\mathrm{F}$ \\
\end{tabular} & $\mathrm{F} F$ & $8 \mathrm{R}$ & $\mathrm{R}$ & & $R$ & c) & A & 96 . & \\
\hline $2-1,119-120$ & 7. 70 & & $R$ & c) 1 & $R F$ & $F / F$ & & A & $\mathrm{c}$ & c & A & $\mathrm{F}$ & $R$ & & $R$ & & F & $F$ & $\mathrm{R}$ & C & & & & R & \begin{tabular}{l|l}
$R$ \\
$R$
\end{tabular} & $\begin{array}{lll}R & F \\
\end{array}$ & $F[F$ & $\begin{array}{l}\mathrm{R} \\
\mathrm{R}\end{array}$ & & & $R$ & c) & $\bar{F}$ & 84. & \\
\hline $2-2.45-46$ & 8. 45 & & \begin{tabular}{|l|l|}
$R$ \\
\end{tabular} & c 1 & R $P$ & $F / F$ & & A & $\mathrm{c}$ & C & A & $F$ & $R$ & & F & $\mathrm{R} / \mathrm{R}$ & F & $\mathrm{F}$ & $\mathrm{R}$ & C & & \begin{tabular}{l|l}
$R$ & $F$ \\
\end{tabular} & $\mathrm{R}$ & $\mathrm{F}$ & R F & $\mathrm{F} F$ & $\begin{array}{ll}\mathrm{R} & \mathrm{B} \\
\mathrm{n}\end{array}$ & $\begin{array}{l}\mathrm{a} \\
\mathrm{R}\end{array}$ & $\mathrm{R}$ & & $R$ & c & $\mathrm{c}$ & 80 & \\
\hline 2-3. $119-120$ & 10. 70 & & \begin{tabular}{|l|l}
$R$ \\
\end{tabular} & c & ts & \begin{tabular}{l|l}
$C$ & $F$ \\
\end{tabular} & & A & $\mathrm{c}$ & c & A & $\mathrm{F}$ & $\mathrm{R}$ & $R$ & $\mathrm{~F}$ & $\mathrm{R}$ & F & $\mathrm{F}$ & $\mathrm{R}$ & c & $R$ & & \begin{tabular}{|l|l}
$R$ \\
\end{tabular} & $R$ & F F & R F & F F & R $R$ & & $\mathrm{R}$ & $R$ & c & $\mathrm{F}$ & $=140$ & \\
\hline $2-4,45-46$ & 11.45 & \begin{tabular}{|l|}
$R$ \\
\end{tabular} & $R$ & c & R & \begin{tabular}{c|c}
$C$ & $F$ \\
\end{tabular} & & A & ac & $c$ & A & $\mathrm{F}$ & $R$ & & & & F & C & $\mathrm{R}$ & c & & & & $\mathrm{R}$ & F F & R F & $F$ F & R $\mathrm{R}$ & $\mathrm{R}$ & & $R$ & $F$ & $\bar{c}$ & 72 . & \\
\hline $2-5.45-46$ & 12. 95 & $R$ & $R$ & $\mathrm{FH}$ & \begin{tabular}{r|r}
$R$ & $F$ \\
$F$
\end{tabular} & $\mathrm{~F} / \mathrm{F}$ & & A & Ac & $F$ & A & c & $F$ & & & & & & $\mathrm{~F}$ & $F$ & & & & $\mathrm{R}$ & $\mathrm{F} F$ & \begin{tabular}{l|l}
$\mathrm{R}$ & $\mathrm{F}$ \\
$\mathrm{n}$
\end{tabular} & $F F$ & $=\mathrm{R}$ & $\mathrm{R}$ & & & $\mathrm{F}$ & $\mathrm{F}$ & 70. & \\
\hline $2-6.45-46$ & 14. 45 & $\mathrm{R}$ & & $\mathrm{F}$ & F & \begin{tabular}{l|l}
$F$ & $F$ \\
\end{tabular} & & A & $F$ & $\mathrm{~F}$ & A & $\mathrm{F}$ & $F$ & $R$ & & & & . & $\mathrm{F}$ & $\mathrm{F}$ & & & & & $F \mathrm{~F}$ & $R \quad F$ & $F / F$ & & $R$ & $R$ & & $F$ & $R$ & 96 & \\
\hline $2 . \mathrm{cc}$ & 16. 10 & $R$ & $R$ & chit & RF & $\mathrm{F} / \mathrm{F}$ & & A & $\mathrm{CC}$ & $c$ & A & $\mathrm{F}$ & R & & $\mathrm{R}$ & & & $\mathrm{R}$ & C & $F$ & & & & $\mathrm{~F}$ & $\mathrm{~F} F$ & \begin{tabular}{l|l}
$R$ & $F$ \\
\end{tabular} & $\mathrm{~F}$ & $\mathrm{~F}$ & $\mathrm{R}$ & & $R$ & c & $\mathrm{F}$ & 72. & \\
\hline $3-1.39-40$ & 16.50 & $R$ & c & $\mathrm{FH}$ & $\mathrm{R} / \mathrm{P}$ & $\mathrm{FCO}$ & $c \mid \mathrm{F}$ & a $v$ & $\mathrm{~F}$ & $F$ & c & c & $F$ & & F & \begin{tabular}{l|l}
$R$ & $R$ \\
\end{tabular} & & $R$ & C & C & & & & $\mathrm{F}$ & \begin{tabular}{l|l} 
C & $F$ \\
\end{tabular} & \begin{tabular}{l|l}
$R$ & $F$ \\
\end{tabular} & $\mathrm{FR}$ & $8 \mid c$ & $F$ & $R$ & $F$ & c & $\mathrm{F}$ & $=200$. & \\
\hline 3-2. $39-40$ & 18.00 & & $F$ & F & $R F$ & $\mathrm{~F} / \mathrm{C}$ & & v & $F$ & $\mathrm{~F}$ & c & c & R & $R$ & & & F & $R$ & C & $F$ & $\mathrm{R}$ & & & $R$ & $F$ F & $F$ F & $F F$ & $=\mathrm{F}$ & $F$ & $\mathrm{~F}$ & $F$ & c & $\mathrm{F}$ & 4667. & \\
\hline $3-3,39-40$ & 19.50 & R. & $F$ & $\mathrm{FF}$ & $\mathrm{R} / \mathrm{F}$ & $\mathrm{F} C \mathrm{C}$ & & $v$ & $1 c$ & $F$ & c & c & $\mathrm{F}$ & $\mathrm{R}$ & & $\mathrm{R}$ & & $\mathrm{R}$ & C & c & $R$ & & & $\mathrm{R}$ & $\mathrm{F} F$ & $\mathrm{~F} F$ & $\mathrm{~F} F$ & $=10$ & $\mathrm{~F}$ & $F$ & $F$ & c & $R$ & c) 300 . & \\
\hline 3-4. $39-40$ & 21.00 & & $\mathrm{R}$ & $\mathrm{F}$ & R F & $\mathrm{F} F$ & & A & $\mathrm{c}$ & C & A & $\mathrm{F}$ & $\mathrm{F}$ & $\mathrm{R}$ & & & & & c & $F$ & & & & $\mathrm{R}$ & $R^{R} F$ & $\begin{array}{lll}R & F \\
\end{array}$ & $\mathrm{~F} F$ & a F & $\mathrm{R}$ & $R$ & $\mathrm{R}$ & c & $\bar{F}$ & 120 & \\
\hline $3-5 . \quad 39-40$ & 22. 50 & & $F$ & Fi & $R / F$ & $F$ F & & A & c & C & c & $F$ & $R$ & & F & $R$ & & & C & $F$ & & & & $\mathrm{R}$ & \begin{tabular}{l|l}
$R$ & $F$ \\
\end{tabular} & R F & $F$ F & $\begin{array}{l}\text { Q } \\
F\end{array}$ & $\mathrm{R}$ & $R$ & $R$ & c) & $\mathrm{F}$ & c) 70. & \\
\hline $3-6.39-40$ & 24.00 & $R$ & $F$ & $\mathrm{FF}$ & $R / F$ & $F / F$ & $=\mathrm{F}$ & R A & C & C & A & c & $F$ & & & F & & R & $F$ & $\mathrm{~F}$ & & & & $\mathrm{R}$ & $\mathrm{F} F$ & $\mathrm{R} F$ & $\mathrm{~F} F$ & Q F & $R$ & & $R$ & $F$ & $\bar{F}$ & 33. & \\
\hline $3 . \mathrm{CC}$ & 25.70 & & c & $\mathrm{F} \mid \mathrm{H}$ & $R / F$ & F C & $C F$ & v & $1 \mathrm{C}$ & A & C & $\mathrm{F}$ & $\mathrm{F}$ & $R$ & & F & F & $R$ & C & C & & & $\mathrm{R}$ & $\mathrm{F}$ & $\mathrm{F} F$ & $F F$ & $\mathrm{~F} F$ & $=F$ & $F$ & $F$ & $\mathrm{~F}$ & $\mathrm{~F}$ & $F$ & c] 533 . & \\
\hline $4-1 \cdot 18-19$ & 25.89 & \begin{tabular}{|l|}
$R$ \\
\end{tabular} & $\mathrm{~F}$ & c|f & 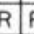 & $\bar{F} F$ & & v & $A$ & A & A & $\mathrm{F}$ & $F$ & $R$ & F & $R$ & & & $\mathrm{~F}$ & $\mathrm{~F}$ & & & & $\mathrm{R}$ & F P & R F & 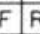 & Q F & R & R & $R$ & $\mathrm{~F}$ & c & c] 100. & \\
\hline $4-2, \quad 18-19$ & 27. 39 & & $R$ & $\mathrm{~F}$ & TF & $\mathrm{F}$ & & A & A & C & A & $\mathrm{F}$ & R & $R$ & & & & & C & F & & & & $R$ & $\mathrm{~F} F$ & \begin{tabular}{l|l}
$R$ & $F$ \\
\end{tabular} & $\mathrm{~F} / \mathrm{R}$ & $\mathrm{R}$ & $R$ & & $R$ & $\mathrm{~F}$ & $F$ & 50 . 50 . & \\
\hline $4-3, \quad 18-19$ & 28.89 & \begin{tabular}{|l|}
$R$ \\
\end{tabular} & $R$ & $\mathrm{~F}$ & $\mathrm{R} F$ & $F F$ & $=R$ & v & C & C & A & c & $R$ & & \begin{tabular}{l|l}
$R$ & $F$ \\
\end{tabular} & $\mathrm{R}$ & & & C & $\mathrm{F}$ & & & & $R$ & $F$ & $F$ & & $F$ & $R$ & R & $R$ & $F$ & C & 200 & \\
\hline $4-4,18-19$ & 30. 39 & & $\mathrm{R}$ & C & $T F$ & $F$ F & & A & A & A & A & c & $R$ & & & $F$ & & & $F$ & $F$ & & & & & $\mathrm{~F}$ & $F$ & $\mathrm{~F} / \mathrm{R}$ & R & $\mathrm{R}$ & & $R$ & $\mathrm{~F}$ & $F$ & 24 & \\
\hline $4-6.125-126$ & 34. 45 & & $R$ & c) & \begin{tabular}{r|r}
$R$ & $F$ \\
$F$
\end{tabular} & $F \mid F$ & & A & A & A & $A$ & $F$ & $R$ & & & & & R & C & $\mathrm{F}$ & & & & $\mathrm{R}$ & \begin{tabular}{l|l}
$R$ & $F$ \\
\end{tabular} & \begin{tabular}{l|l}
$R$ & $F$ \\
\end{tabular} & $\mathrm{~F}$ & $\mathrm{R}$ & & $\mathrm{R}$ & $R$ & $\mathrm{~F}$ & $\mathrm{~F}$ & 35. & \\
\hline 4. $\mathrm{CC}$ & 35. 30 & \begin{tabular}{|l|}
$R$ \\
\end{tabular} & \begin{tabular}{l|l}
$R$ \\
\end{tabular} & c| & $R F$ & $F$ F & & A & $A$ & A & A & $\mathrm{F}$ & $\mathrm{F}$ & & \begin{tabular}{l|l}
$R$ & $F$ \\
\end{tabular} & $R$ & $R$ & & C & $\mathrm{F}$ & & & & $\mathrm{R}$ & $\mathrm{F} F$ & $F F$ & $\mathrm{~F} / \mathrm{R}$ & 2 R & $R$ & $R$ & $F$ & $\mathrm{~F}$ & $\bar{F}$ & 20. & \\
\hline $5-1 \cdot 9-10$ & 35.40 & $\mathrm{R}$ & $R$ & c & $T_{\mathrm{F}}$ & $\bar{F} F$ & & A & $A$ & C & A & $F$ & $\bar{R}$ & & $\mathrm{R}$ & & & & C & $F$ & & & & $\mathrm{R}$ & $\mathrm{F} F$ & R F & \begin{tabular}{l|l}
$R$ & $R$ \\
\end{tabular} & Q. $F$ & $\mathrm{R}$ & & $R$ & c & $\bar{c}$ & 62.5 & \\
\hline $5-2.9-10$ & 36.90 & & $F$ & $\mathrm{~F}$ & \begin{tabular}{l|l}
$R$ & $F$ \\
\end{tabular} & $\mathrm{~F}$ & & v & C & $F$ & A & $F$ & $R$ & & $R$ & & & $R$ & C & C & & & & $\mathrm{F}$ & $\mathrm{F} F$ & $\mathrm{~F} F$ & $F \mid F$ & $=10$ & $F$ & $\mathrm{~F}$ & $F$ & c) & F & 250 & \\
\hline $5-3,9-10$ & 38.40 & & \begin{tabular}{|l|l|}
$R$ \\
\end{tabular} & $\mathrm{~F}$ & F & $C F$ & $=R$ & $\mathrm{v}$ & $1 \mathrm{C}$ & $F$ & A & $\mathrm{F}$ & R & $\mathrm{R} / \mathrm{R}$ & $R$ & F & & & C & c & R & & & $\mathrm{F}$ & $\mathrm{F} F$ & $F \mid F$ & $F$ & $\mathrm{~F}$ & $\mathrm{R}$ & R & $F$ & c| & $=$ & 600. & \\
\hline $5-4,9-10$ & 39. 96 & & $R$ & $F$ & $\mathrm{FF}$ & $F F$ & & A & $c$ & $F$ & $A$ & $F$ & $F$ & & & $F$ & & & C & $\mathrm{F}$ & & & & $\mathrm{R}$ & $\mathrm{F} F$ & $\begin{array}{ll}R & F \\
\end{array}$ & $\mathrm{~F} / \mathrm{R}$ & \begin{tabular}{l|l} 
& $F$ \\
\end{tabular} & & & $R$ & $\mathrm{~F}$ & c & 36 . & \\
\hline $5-6,9-10$ & 42. 90 & \begin{tabular}{|l|}
$R$ \\
\end{tabular} & $R$ & F & F F & $F$ F & & A & $\mathrm{C}$ & $F$ & c & $\mathrm{F}$ & $R$ & & & & $\mathrm{R}$ & & $F$ & $F$ & & & & R & F F & \begin{tabular}{l|l}
$R$ & $F$ \\
\end{tabular} & $\mathrm{~F} / \mathrm{R}$ & Q $\mathrm{F}$ & R & & $R$ & $\mathrm{~F}$ & $\bar{c}$ & 28. & \\
\hline $5 . \mathrm{CC}$ & 44.90 & $R$ & $R$ & $\mathrm{~F}$ & $\mathrm{~F} F$ & $F \mid F$ & & A & C & $F$ & c & $R$ & R & $\mathrm{R}$ & & & & & $F$ & $\mathrm{~F}$ & & & & R & \begin{tabular}{l|l}
$R$ & $F$ \\
\end{tabular} & \begin{tabular}{l|l}
$R$ & $F$ \\
\end{tabular} & $\mathrm{R}$ & $\mathrm{F}$ & $R$ & $R$ & $R$ & $\mathrm{~F}$ & $F$ & 20. & \\
\hline $6-1.9-10$ & 45.00 & & & $\mathrm{~F}$ & $\begin{array}{ll}\mathrm{R} \\
\mathrm{B}\end{array}$ & $\mathrm{F} / \mathrm{F}$ & & A & c & $F$ & $A$ & $\mathrm{~F}$ & R & $R \mid$ & $R$ & & & & $F$ & $\mathrm{~F}$ & & & & R & $\begin{array}{lll}R & F \\
\end{array}$ & \begin{tabular}{l|l}
$R$ & $F$ \\
\end{tabular} & \begin{tabular}{l|l}
$R$ & $R$ \\
\end{tabular} & $R$ & $\mathrm{R}$ & $R$ & $R$ & $\mathrm{~F}$ & c & 32. & \\
\hline $6-2,9-10$ & 46. 50 & $\mathrm{R}$ & $R$ & $\mathrm{~F}$ & \begin{tabular}{l|l} 
\\
$R$
\end{tabular} & $\bar{F} F$ & & A & A & $A$ & $A$ & $R$ & $t i$ & $\begin{array}{lll}R & 1\end{array}$ & $R$ & & & & C & $\mathrm{F}$ & & & & R & $F F$ & $\begin{array}{ll}R & F \\
\end{array}$ & \begin{tabular}{l|l}
$R$ & $R$ \\
\end{tabular} & R $\mathrm{R}$ & & $R$ & $R$ & $\mathrm{~F}$ & c & 20. & \\
\hline $6-3,9-10$ & 48.00 & & & c| & $R$ & $F / F$ & & c & A & c & $A$ & $\mathrm{~F}$ & R & & F & $\mathrm{R}$ & & & $\mathrm{F}$ & $\mathrm{F}$ & & & & R & $\begin{array}{ll}R & F \\
\end{array}$ & $\begin{array}{ll}R & F \\
\end{array}$ & \begin{tabular}{l|l}
$\mathrm{R}$ & $\mathrm{R}$ \\
\end{tabular} & \begin{tabular}{l|l} 
& $R$ \\
\end{tabular} & & $R$ & & $\mathrm{~F}$ & $F$ & 27. & \\
\hline $6-4 \cdot 9-10$ & 49. 50 & $\mathrm{R}$ & $R$ & $F$ & F & $F F$ & $=R$ & A & C & $F$ & A & $F$ & R & R & $R$ & & & $R$ & $\mathrm{~F}$ & $\mathrm{~F}$ & & $R$ & & $R$ & $F$ & $R F$ & $\mathrm{~F} / \mathrm{R}$ & $R$ & $\mathrm{R}$ & $R$ & $R$ & $F$ & c & 32. & \\
\hline $6 . \mathrm{cc}$ & 50.90 & & $R$ & $\mathrm{~F}$ & F & F F & & 0 & C & C & c & $\mathrm{F}$ & & & R & & & & $F$ & $\mathrm{~F}$ & & & & & $F F$ & \begin{tabular}{l|l}
$R$ & $F$ \\
\end{tabular} & & $\mathrm{R}$ & $\mathrm{R}$ & & $R$ & $\mathrm{~F}$ & c & 24. & \\
\hline $7-1.9-10$ & 51,00 & & $F$ & $\mathrm{~F}$ & $F$ & $F$ F & $=R$ & c & C & $F$ & c & $\mathrm{R}$ & $R$ & & & F & & & $F$ & $F$ & & & & R & $\mathrm{F} F$ & \begin{tabular}{l|l}
$R$ & $F$ \\
\end{tabular} & $R$ & R & & & $R$ & $\mathrm{~F}$ & 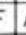 & 18. & \\
\hline $7-2,9-10$ & 52.50 & & $R$ & c & F & \begin{tabular}{l|l}
$F$ & $F$ \\
\end{tabular} & $=R$ & A & C & C & $A$ & $\mathrm{~F}$ & $R$ & & \begin{tabular}{l|l}
$R$ & $F$ \\
\end{tabular} & R & & & $F$ & $\mathrm{~F}$ & & & & R & $\mathrm{F} F$ & $\begin{array}{ll}R & F \\
\end{array}$ & \begin{tabular}{l|l}
$F$ & $R$ \\
\end{tabular} & \begin{tabular}{l|l}
$R$ \\
$R$
\end{tabular} & & & $R$ & $\mathrm{~F}$ & c & 24. & \\
\hline $7-3,9-10$ & 5400 & \begin{tabular}{|l|}
$R$ \\
\end{tabular} & $R$ & c| & $R F$ & $\bar{F} F$ & & $A$ & C & $\mathrm{F}$ & $A$ & $F$ & $R$ & & $R$ & & & $R$ & $\mathrm{~F}$ & $F$ & & & & $\mathrm{R}$ & $F F$ & $R \mid F$ & \begin{tabular}{l|l}
$F$ & $R$ \\
\end{tabular} & & R & & $R$ & $\mathrm{~F}$ & $\bar{F}$ & 27. & \\
\hline $7-4,9-10$ & 55.50 & $\mathrm{R}$ & $R$ & Fi & \begin{tabular}{l|l}
$\mathrm{R}$ & $\mathrm{F}$ \\
\end{tabular} & $\mathrm{F} / \mathrm{F}$ & $=R$ & A & c & $F$ & $A$ & $\mathrm{~F}$ & & & & & & R & $\mathrm{F}$ & $\mathrm{F}$ & & & & $\mathrm{R}$ & $\mathrm{R}$ & F & $\mathrm{R} \mid$ & $\mathrm{R}$ & $\mathrm{R}$ & R & & $\mathrm{F}$ & $\bar{F}$ & 27. & \\
\hline $8-1,30-31$ & 57.21 & & $\mathrm{~F}$ & F & R F & F F & \begin{tabular}{l|l} 
\\
$R$
\end{tabular} & A & C & $\mathrm{F}$ & c & $\mathrm{F}$ & & & & & & & $\mathrm{F}$ & $R$ & & & t & R & $\mathrm{F} F$ & \begin{tabular}{l|l}
$R$ & $F$ \\
\end{tabular} & $\mathrm{~F}$ & $R$ & $\mathrm{R}$ & & $R$ & $\mathrm{~F}$ & 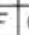 & 30 & \\
\hline $8-2.30-31$ & 5871 & & $R$ & R & R F & F F & $=R$ & A & C & $\mathrm{F}$ & c & $\mathrm{F}$ & & & R & & & & $\mathrm{F}$ & $F$ & & & & $\mathrm{R}$ & $F F$ & $R \mid F$ & $\mathrm{~F}$ R & $\begin{array}{l}Q \\
R\end{array}$ & & & $R$ & $\mathrm{~F}$ & c & 21. & \\
\hline $8-3 \cdot 30-31$ & 60.21 & & \begin{tabular}{|l|l|}
$R$ \\
\end{tabular} & F & $R$ & $F / F$ & & A & C & $F$ & A & $\mathrm{F}$ & $R$ & & & F & & $R$ & $\mathrm{~F}$ & $\mathrm{~F}$ & & & & R & $\mathrm{F} / \mathrm{F}$ & $\mathrm{F} \mid \mathrm{F}$ & $\mathrm{F} / \mathrm{R}$ & R & $R$ & & $R$ & $\mathrm{~F}$ & R & 27. & \\
\hline $8-5,30-31$ & 63.21 & & $R$ & $\mathrm{~F}$ & Te & \begin{tabular}{c|c|c|}
$c$ &
\end{tabular} & $\begin{array}{l}\mathrm{R} \\
\mathrm{s}\end{array}$ & A & C & $F$ & c & $\mathrm{F}$ & R & & & & & & $\mathrm{F}$ & $\mathrm{F}$ & & & & R & $F F$ & \begin{tabular}{l|l}
$\mathrm{R}$ & $\mathrm{F}$ \\
\end{tabular} & $F R$ & $R$ & & $R$ & $R$ & $\mathrm{~F}$ & R & 36. & \\
\hline 8.CC & 63.50 & & & $R$ & & & & $F$ & $R$ & $R$ & $\mathrm{~F}$ & & & & & & & & $R$ & & & & & & & & & & & & & & 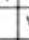 & 04 & \\
\hline $9-1.29-30$ & 70.20 & & $R$ & FII & R. & \begin{tabular}{c|c}
$c$ & $F$ \\
\end{tabular} & & A & C & $F$ & A & $\mathrm{F}$ & R & $R$ & & & & & $F$ & $\mathrm{~F}$ & & & $r$ & $\mathrm{R} \mid$ & $\mathrm{F} F$ & \begin{tabular}{l|l}
$R$ & $F$ \\
\end{tabular} & $\mathrm{~F} R$ & R & & $R$ & $R$ & $\mathrm{~F}$ & F & 48 & \\
\hline $9-2, \quad 29-30$ & 71.70 & $\mathrm{R}$ & $R$ & FI & RF & $\begin{array}{l}F \\
F\end{array}$ & & A & C & $F$ & A & $\mathrm{F}$ & R & $\mathrm{R} / \mathrm{F}$ & $R$ & & & & $\mathrm{~F}$ & $\mathrm{~F}$ & & & $f$ & $\mathrm{R}$ & $F F$ & \begin{tabular}{l|l}
$\mathrm{R}$ & $\mathrm{F}$ \\
\end{tabular} & $\mathrm{F} / \mathrm{R}$ & $\mathrm{R}$ & R & & $R$ & $\mathrm{~F}$ & c & 20 & \\
\hline $9 . c c$ & 74.40 & & $R$ & Fi & $R$ & F F & & A & C & C & $A$ & $\mathrm{~F}$ & R & R & F & $\mathrm{R}$ & & & $F$ & $\mathrm{~F}$ & & & f & R & $F F$ & $\mathrm{R} / \mathrm{F}$ & $\mathrm{F}$ & $R$ & $\mathrm{R}$ & & $R$ & $\mathrm{~F}$ & 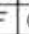 & 40 & \\
\hline $10-1,66-67$ & 75.07 & & & $\mathrm{~F}$ & F & $F$ F & & c & C & $F$ & c| & $\mathrm{F}$ & \begin{tabular}{l|l} 
\\
$R$
\end{tabular} & & R & & F & & F & $\mathrm{F}$ & & & & R & $F$ & \begin{tabular}{l|l}
$R$ & $F$ \\
\end{tabular} & $R F$ & $\mathrm{R}$ & $\mathrm{R}$ & & $R$ & $\mathrm{~F}$ & 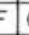 & 40 & \\
\hline $10-2 . \quad 48-49$ & 76. 40 & & $R$ & $\mathrm{~F}$ & RP & $F F$ & & A & C & $\mathrm{F}$ & $A$ & $F$ & R & & & & & & $F$ & $F$ & & & & R & $\mathrm{R} F$ & $\begin{array}{lll}R & F\end{array}$ & $F F$ & R & & R & $R$ & $F$ & $=$ & 40 & \\
\hline $10-4, \quad 66-67$ & 79.57 & & $R$ & $\mathrm{~F}$ & $\mathrm{~F}$ & F & $=R$ & 8 & C & $F$ & c & $\mathrm{F}$ & fi & 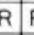 & $\mathrm{R}$ & & & R & $\mathrm{F}$ & $\mathrm{F}$ & & & & R & $\mathrm{R}$ & F & $\mathrm{FF}$ & s. & $\mathrm{R}$ & & $R$ & $\mathrm{~F}$ & $E$ & 30. & \\
\hline $10 . \mathrm{CC}$ & 81, 00 & & \begin{tabular}{|l|l|}
$R$ \\
\end{tabular} & $\mathrm{~F}$ & F & $\mathrm{F} F$ & & $A$ & A & $\mathrm{F}$ & A & $\mathrm{F}$ & R & & & & & & $\mathrm{F}$ & C & & & & R & $\mathrm{F}$ & $\begin{array}{ll}\mathrm{R} & \mathrm{F} \\
\end{array}$ & F $F$ & $R$ & $\mathrm{R}$ & & $R$ & $\mathrm{~F}$ & 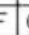 & 40 & \\
\hline $11-1,3-4$ & 89. 14 & & $R$ & $\mathrm{~F}$ & $F$ & $\mathrm{~F} F$ & & c & A & $\mathrm{F}$ & $A$ & $\mathrm{~F}$ & $R$ & F & $R$ & & & & $\mathrm{~F}$ & $\mathrm{~F}$ & & & r & R & $\mathrm{F} F$ & R F & $F$ F & $\mathrm{R}$ & & & $R$ & $\mathrm{~F}$ & $=$ & 36 & \\
\hline $11-2,3-4$ & 90.60 & $\mathrm{R}$ & & $\bar{c}$ & F & $F F$ & & A & $A$ & $\mathrm{~F}$ & $\mathrm{v}$ & $\mathrm{F}$ & & & $R$ & & & $\mathrm{R}$ & $\mathrm{F}$ & $\mathrm{F}$ & & & 1 & R & R F & R $\mathrm{F}$ & R & $\mathrm{R}$ & $\mathrm{R}$ & & & $\mathrm{F}$ & 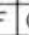 & 30. & \\
\hline $11 . c c$ & 92.50 & $R$ & $R$ & & & $F F$ & & A & & $\mathrm{F}$ & c & $\mathrm{F}$ & $R$ & & F & $R$ & & & & $\mathrm{~F}$ & & & & & $\mathrm{~F} F$ & R F & $F F$ & $R$ & & R & $R$ & $\mathrm{~F}$ & & 24. & \\
\hline
\end{tabular}

Note: For abundance, $V=$ very abundant, $10-100$ specimens/field; $A=$ abundant, $1-10$ specimens/field; $C=$ common, 1 specimen $/ 2-10$ fields; $F=$ few, 1 specimen $/ 11-100$ fields; $R=$ rare, 1 specimen $/ 101-1000$ fields (when viewed at a magnification of $\times 1250$ ). 
nofossils of early Pliocene age. The fairly diverse nannofossil flora includes $D$. pentaradiatus, $D$. brouweri, $S$. abies, Reticulofenestra pseudoumbilica, and $D$. tamalis and is assignable to Okada and Bukry's (1980) $R$. pseudoumbilica Zone (CN11), equivalent to NN15 of Martini (1971). The common co-occurrence of Pseudoemiliania lacunosa, Gephyrocapsa oceanica, and other gephyrocapsids in these clasts suggest that they may be early Pleistocene in age, with a common reworked Pliocene constituent.

The calculated values of the in situ/reworked ratio are considerably higher than in the Mississippi Fan (Fig. 5 ). The sawtooth pattern above $41 \mathrm{~m}$ is a result of the abundance of indigenous calcareous nannofossils in the black clay layers. The ratio in the remainder of the hole is relatively constant resulting from the overall large amount of reworking and mixing of sediments. Active diapirism and instability of the basin slope sediments

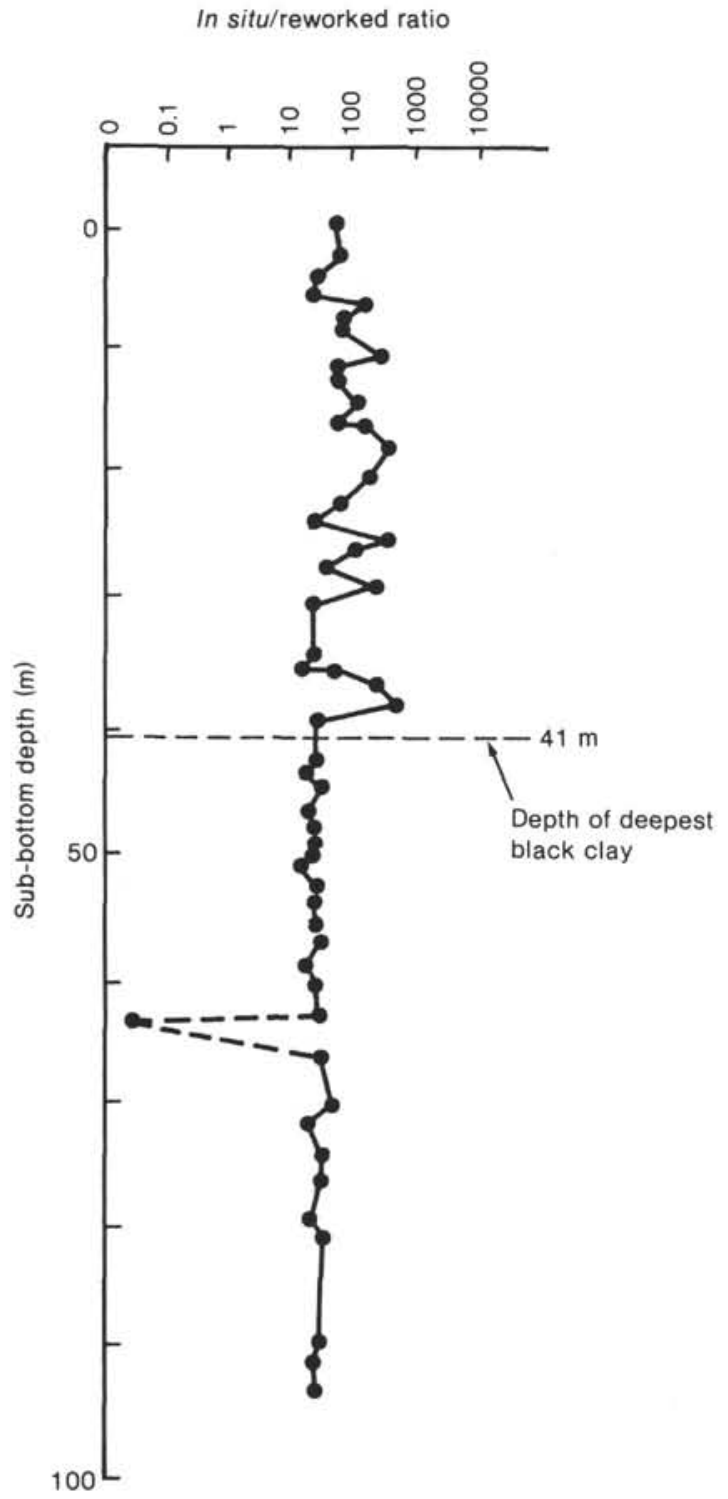

Figure 5. In situ/reworked ratio curve for Site 618. may be responsible for the considerable amount of reworking at this site.

\section{Site 619}

Site $619\left(27^{\circ} 11.61^{\prime} \mathrm{N}, 91^{\circ} 24.54^{\prime} \mathrm{W}\right.$; water depth, $2274 \mathrm{~m}$ ) is located near the axis of Pigmy Basin, a blocked-canyon intraslope basin on the Louisiana middle continental slope. An almost complete clay sequence of Holocene and Wisconsin (Ericson Zones, Z, Y, and $\mathrm{X})$ sediments was recovered to the total penetration depth of $208.7 \mathrm{~m}$ sub-bottom. Early Wisconsin glacial (Ericson Zone W) sediments may have been encountered in Section 619-18-2 through Sample 619-25,CC, but the biostratigraphic placement of this interval is still interpretive at the time of this writing (Kohl, this volume; Williams and Kohl, this volume).

Moderately well-preserved nannofossils are generally abundant throughout all samples at this site (Table 11). They are particularly abundant in the intervals from Sections 619-1-1 through 619-1-3 and from Cores 619-15 through 619-18. Reworked Cretaceous taxa are relatively rare, especially in comparison with their almost total domination of the assemblages in the Mississippi Fan sites. Samples from 619-1-1, 0-1 cm through 619-16-4, 24-25 $\mathrm{cm}$ are placed in the E. huxleyi Acme Zone of Gartner and Emiliani, while the interval from Samples 619-16,CC through $619-25, C C$ is placed in the $E$. huxleyi Zone. The boundary between these zones, marked by the reversal in dominance of E. huxleyi and Gephyrocapsa spp., is placed in Sample 619-16,CC. While Gephyrocapsa spp. is only marginally dominant in this sample, it is clearly dominant in Sample 619-17-1, 30-31 cm. In Sample 619$16-4,24-25 \mathrm{~cm}, E$. huxleyi is dominant.

In tropical and subtropical waters, the reversal in dominance of E. huxleyi and Gephyrocapsa spp. is associated with oxygen-isotope Substage $5 \mathrm{~b}-5 \mathrm{a}$, approximately 85,000 yr. ago (Thierstein et al., 1977). Anderson and Steinmetz (1983) reconfirm this relationship in the late Quaternary Caribbean Core P6304-4. Williams and Kohl (this volume) also place the deepest occurrence of dominant $E$. huxleyi in oxygen-isotope Substages 5b-5a.

A volcanic ash layer, also found in Sample 619-16,CC, has been identified as the Y8 ash of Kennett and Huddleston (1972) and is dated at $84,000 \mathrm{yr}$. ago (Ledbetter, this volume). The deepest dominant occurrence of $E$. huxleyi in Site 619 can therefore be most accurately dated by both stable isotope and tephrochronology as occurring in isotope Stage $5 \mathrm{~b}-5 \mathrm{a}$ at $84,000 \mathrm{yr}$. ago.

\section{DISCUSSION}

The amount of terrigenous detrital material available for deposition in the deep water sites of Leg 96 is controlled by glacio-eustatic fluctuations. During interglacial high stands of sea level, much of the sediment is trapped on the broad continental shelf. The lowering of sea level during glacial periods is responsible for an increase in the amount of detritus because of increased stream gradients, sediment instability, and an overall narrowing of the continental shelf. A detailed discussion of the factors controlling this relationship can be found in 
Table 11. Distribution of calcareous nannofossils, Site 619.

\begin{tabular}{|c|c|c|c|c|c|c|c|c|c|c|c|c|c|c|c|c|c|c|c|c|c|c|c|c|c|c|c|c|c|c|c|c|}
\hline $\begin{array}{l}\text { Core- } \\
\text { Section } \\
\text { (interval } \\
\text { in cm) }\end{array}$ & \begin{tabular}{|c} 
Sub- \\
bottom \\
depth \\
(m)
\end{tabular} & & & & & & & 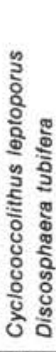 & & & & & & & 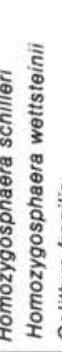 & & 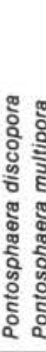 & & & 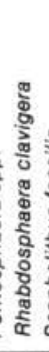 & 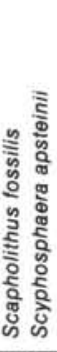 & & & 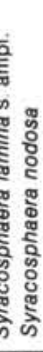 & & 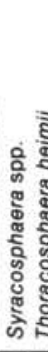 & & & & & 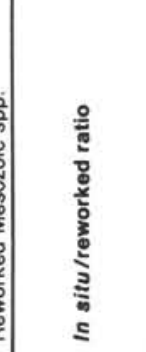 & $\begin{array}{l}\text { Zone or } \\
\text { subzone }\end{array}$ \\
\hline $1-1.0-1$ & .01 & $R$ & \begin{tabular}{|l|l}
$R$ \\
\end{tabular} & $\mathrm{~F} / \mathrm{C}$ & $\begin{array}{ll}C & R \\
\end{array}$ & \begin{tabular}{|l|} 
\\
\end{tabular} & \begin{tabular}{|l|l}
$c$ \\
\end{tabular} & \begin{tabular}{l|l}
$F$ & $F$ \\
\end{tabular} & [H] & $\mathrm{F} / \mathrm{C}$ & $R$ & $F$ & \begin{tabular}{l|l|}
$C$ & $R$ \\
\end{tabular} & $F R$ & \begin{tabular}{l|l} 
a & \\
\end{tabular} & \begin{tabular}{l|l}
$R$ & $F$ \\
\end{tabular} & & \begin{tabular}{l|l|l|}
$R$ & $F$ \\
\end{tabular} & \begin{tabular}{|l|l|}
$R$ & $F$ \\
\end{tabular} & C & \begin{tabular}{l|l|}
$C$ & $R$ \\
\end{tabular} & $\mathrm{R}$ & $F \mid F$ & $F$ & $\mathrm{R}$ & $\mathrm{F}$ & $\mathrm{R}$ & \begin{tabular}{|l|l|}
$F$ & $F$ \\
\end{tabular} & C & $\mathrm{F} C \mathrm{C}$ & 1500. & \multirow{63}{*}{$\begin{array}{c}\text { Emiliania } \\
\text { huxieyi } \\
\text { acme }\end{array}$} \\
\hline $1-1,27-28$ & .27 & & or & $F \mid F$ & \begin{tabular}{l|l}
$F$ & $R$ \\
\end{tabular} & & \begin{tabular}{|l|l}
$F$ \\
\end{tabular} & \begin{tabular}{l|l}
$C$ & $F$ \\
\end{tabular} & $\mathrm{H}$ & $\mathrm{F} C \mathrm{C}$ & $R$ & \begin{tabular}{l|l|l}
$F$ & $F$ \\
\end{tabular} & F & $F$ & $R$ & & R & $F$ F & $\begin{array}{ll}R & R \\
\end{array}$ & c & \begin{tabular}{l|l|}
$c$ & \\
\end{tabular} & $\mathrm{R}$ & $F \mid F$ & $F$ & $F$ & \begin{tabular}{|l|l|}
$F$ & $F$ \\
\end{tabular} & $F$ & \begin{tabular}{|l|l|}
$R$ & \\
\end{tabular} & c & $\mathrm{RCC}$ & 625. & \\
\hline $1-2.27-28$ & 2.77 & & $\begin{array}{ll}R \\
\end{array}$ & $F \mid C$ & c & & $F$ & \begin{tabular}{|l|l|}
$F$ & $F$ \\
\end{tabular} & $\mathrm{v}$ & \begin{tabular}{l|l}
$c$ & $c$ \\
\end{tabular} & & $\begin{array}{lll}C & 0 \\
\end{array}$ & c & \begin{tabular}{l|l}
$F$ & $R$ \\
\end{tabular} & & \begin{tabular}{l|l}
$\mathrm{R}$ & $\mathrm{F}$ \\
$\mathrm{H}$
\end{tabular} & \begin{tabular}{l|l}
$R$ & $R$ \\
\end{tabular} & $R$ & $\mathrm{R}$ & c & c & & $F / F$ & $F$ & c & $\mathrm{F} F$ & $R$ & \begin{tabular}{l|l}
$R$ & $F$ \\
\end{tabular} & C & $\begin{array}{lll}R & A \\
\end{array}$ & 250. & \\
\hline $1-3,27-28$ & 4. 27 & & & $F \mid A$ & A & & \begin{tabular}{|l|l|l|}
$F$ & $F$ \\
\end{tabular} & \begin{tabular}{l|l}
$F$ & $F$ \\
\end{tabular} & H & \begin{tabular}{l|l}
$c$ & $c$ \\
\end{tabular} & & $F \mid C$ & c & $\mathrm{F}$ & & \begin{tabular}{l|l}
$R$ \\
\end{tabular} & R & $R$ & $\mathrm{R}$ & c & C & & $\mathrm{F} \mid \mathrm{C}$ & $F$ & c & $F \mid F$ & $R$ & \begin{tabular}{l|l}
$R$ & $F$ \\
\end{tabular} & C & $R C$ & 500. & \\
\hline 1-4. $27-28$ & 5. 78 & $\mathrm{R}$ & \begin{tabular}{|l|l|l} 
\\
\end{tabular} & $F \mid C$ & c & & $F$ & \begin{tabular}{|l|l|}
$F$ & $F$ \\
\end{tabular} & $\mathrm{v}$ & \begin{tabular}{l|l} 
c & C
\end{tabular} & & \begin{tabular}{|l|l|}
$F$ & $F$ \\
\end{tabular} & & $F$ & & \begin{tabular}{l|l}
$R$ & $F$ \\
$R$
\end{tabular} & \begin{tabular}{l|l} 
R \\
\end{tabular} & $R$ & $R$ & c & c & & $F / F$ & $F$ & c & \begin{tabular}{l|l}
$F$ & $F$ \\
\end{tabular} & $R$ & \begin{tabular}{l|l}
$R$ & $F$ \\
\end{tabular} & C & $\begin{array}{ll}c \\
\end{array}$ & 69. & \\
\hline $1-5.27-28$ & 7. 78 & $\mathrm{R}$ & & $F \mid A$ & A & & \begin{tabular}{l|l}
$F$ \\
\end{tabular} & \begin{tabular}{l|l}
$F$ & $F$ \\
\end{tabular} & $\mathrm{v}$ & \begin{tabular}{l|l}
$c$ & $c$ \\
\end{tabular} & & \begin{tabular}{|l|l|l|}
$F$ & $F$ \\
\end{tabular} & & $F$ & & & $\mathrm{R}$ & & $\mathrm{R}$ & c & c & & \begin{tabular}{l|l}
$R$ & $F$ \\
\end{tabular} & & c & \begin{tabular}{l|l}
$F$ \\
$F$
\end{tabular} & $F$ & \begin{tabular}{l|l}
$R$ & $F$ \\
\end{tabular} & C & $\mathrm{R}$ & 60. & \\
\hline $1-6.27-28$ & 8. 78 & & & $F$ & $F$ & & $R$ & + & $F$ & & & \begin{tabular}{l|l}
$R$ & $F$ \\
\end{tabular} & $R$ & & & & & 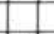 & 1 & D & $R$ & $R$ & & 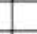 & + & & $R$ & & $R$ & A & 1. & \\
\hline $2 . c c$ & 1.00 & $\mathrm{R}$ & & $F A$ & A & & \begin{tabular}{l|l}
$F$ & $F$ \\
\end{tabular} & \begin{tabular}{|l|l|}
$F$ & $F$ \\
\end{tabular} & $\mathrm{H}$ & \begin{tabular}{l|l}
$c$ & $c$ \\
\end{tabular} & & $F$ & $\mathrm{~F}$ & C & & \begin{tabular}{l|l}
$R$ & $F$ \\
\end{tabular} & $R$ & $\mathrm{R}$ & $\mathrm{R}$ & c & c & & \begin{tabular}{|l|l|l}
$F$ & $F$ \\
\end{tabular} & $F$ & c| & \begin{tabular}{l|l}
$F$ & $F$ \\
\end{tabular} & $F$ & \begin{tabular}{|l|l|}
$R$ & $F$ \\
\end{tabular} & c & $\begin{array}{lll}R & C \\
\end{array}$ & 500. & \\
\hline $3-1.24-25$ & 10.75 & & & 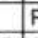 & \begin{tabular}{l|l}
$R$ \\
\end{tabular} & & \begin{tabular}{l|l} 
\\
\end{tabular} & & $F$ & & & F F & $R$ & & & & & & & & & & & & & $R$ & & \begin{tabular}{|l|l|} 
& \\
\end{tabular} & & 10 & 5 & \\
\hline $3-2,24-25$ & 12. 25 & & R & $R \mid C$ & \begin{tabular}{c|c}
$c$ & $F$ \\
\end{tabular} & & F & \begin{tabular}{l|l}
$F$ & $R$ \\
\end{tabular} & $\mathrm{v}$ & $\begin{array}{ll}c & C \\
\end{array}$ & & \begin{tabular}{|l|l}
$C$ & $F$ \\
\end{tabular} & $\mathrm{~F}$ & $R$ & & \begin{tabular}{l|l}
$R$ & $F$ \\
\end{tabular} & $F$ & \begin{tabular}{l|l}
$R$ & $F$ \\
\end{tabular} & \begin{tabular}{l|l|}
$R$ & $F$ \\
\end{tabular} & $F$ & $F$ & & \begin{tabular}{|l|l|}
$F$ & $F$ \\
\end{tabular} & $F$ & c & \begin{tabular}{l|l}
$F$ \\
$F$
\end{tabular} & $R$ & \begin{tabular}{|l|l|}
$F$ & $R$ \\
\end{tabular} & c & $C A$ & 42. & \\
\hline 3-3. $24-25$ & 13.75 & & & $\mathrm{~F} \mid \mathrm{C}$ & \begin{tabular}{l|l}
$c$ & $F$ \\
\end{tabular} & & F & \begin{tabular}{l|l}
$F$ & $R$ \\
\end{tabular} & $\mathrm{v}$ & $\begin{array}{lll}c & C \\
\end{array}$ & & \begin{tabular}{l|l} 
c & ( \\
\end{tabular} & c & $F$ & & \begin{tabular}{l|l}
$R$ & $F$ \\
\end{tabular} & \begin{tabular}{l|l}
$F$ & $R$ \\
\end{tabular} & \begin{tabular}{|l|l|}
$R$ & $F$ \\
\end{tabular} & \begin{tabular}{l|l|}
$R$ & $R$ \\
\end{tabular} & $F$ & c & $\mathrm{R}$ & $F \mid F$ & $R$ & c & $F F$ & $R$ & \begin{tabular}{|l|l|}
$F$ & $F$ \\
\end{tabular} & $c$ & F & 50. & \\
\hline $3-4, \quad 24-25$ & 15. 25 & & & $\mathrm{~F} C \mathrm{C}$ & \begin{tabular}{l|l}
$c$ & $R$ \\
\end{tabular} & & $F \mid$ & \begin{tabular}{l|l}
$F$ & $R$ \\
\end{tabular} & $\mathrm{v}$ & \begin{tabular}{l|l}
$c$ & $c$ \\
\end{tabular} & & \begin{tabular}{l|l} 
C & ( \\
\end{tabular} & c & $R$ & & & \begin{tabular}{l|l} 
R \\
\end{tabular} & $F$ F & \begin{tabular}{l|l|}
$R$ & $R$ \\
\end{tabular} & c & c & & \begin{tabular}{l|l}
$R$ & $F$ \\
\end{tabular} & $F$ & $F$ & \begin{tabular}{l|l}
$c$ & $F$ \\
\end{tabular} & $F$ & \begin{tabular}{|l|l|}
$F$ & $F$ \\
\end{tabular} & $c$ & $R C$ & 100. & \\
\hline $3-5,24-25$ & 16.75 & & & $\mathrm{FCC}$ & \begin{tabular}{c|c} 
c \\
c
\end{tabular} & & $F$ & & v & \begin{tabular}{l|l}
$F$ \\
\end{tabular} & & \begin{tabular}{l|l}
$c$ & $F$ \\
\end{tabular} & $F$ & $F$ & & & $\begin{array}{l}\mathrm{R} \\
\end{array}$ & $R$ & & $F$ & $F$ & & \begin{tabular}{|l|l}
$F$ & $F$ \\
\end{tabular} & $R$ & $F$ & $\mathrm{~F} F$ & $R$ & \begin{tabular}{l|l}
$R$ & $R$ \\
\end{tabular} & $F$ & T & 22. & \\
\hline $3-6.24-25$ & 18.25 & & & $\begin{array}{lll}R & C \\
\end{array}$ & c & & F & $R$ & $\mathrm{v}$ & $\begin{array}{l}\mathrm{F} F \\
\mathrm{~F}\end{array}$ & & \begin{tabular}{l|l}
$F$ & $F$ \\
\end{tabular} & $\mathrm{~F}$ & $F$ & & & \begin{tabular}{l|l}
$\mathrm{R}$ & $\mathrm{R}$ \\
\end{tabular} & $\mathrm{R}$ & $\mathrm{R}$ & $R$ & $\mathrm{~F}$ & & \begin{tabular}{l|l}
$R$ & $F$ \\
\end{tabular} & $R$ & $F$ & \begin{tabular}{l|l}
$F$ & $F$ \\
\end{tabular} & $R$ & \begin{tabular}{l|l}
$R$ & $R$ \\
\end{tabular} & $R$ & 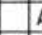 & 6. & \\
\hline 3. $c c$ & 19.33 & & i & $F \mid A$ & A & & F & $\mathrm{R}$ & $\mathrm{v}$ & $\mathrm{F} F$ & & \begin{tabular}{l|l}
$F$ & $F$ \\
\end{tabular} & $\mathrm{~F}$ & $F$ & & & $\mathrm{R}$ & & $\mathrm{A}$ & $F$ & $\mathrm{~F}$ & & \begin{tabular}{l|l}
$R$ & $F$ \\
\end{tabular} & $R$ & $F$ & \begin{tabular}{l|l}
$F$ & $F$ \\
\end{tabular} & $R$ & \begin{tabular}{l|l}
$R$ & $R$ \\
\end{tabular} & $F$ & $\mathrm{R}$ & 12. & \\
\hline $4-1,24-25$ & 20.44 & & & $R \mid A$ & A & & $F$ & & A & 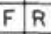 & & \begin{tabular}{l|l}
$F$ & $F$ \\
\end{tabular} & $F$ & $R$ & & & & & $A$ & $R$ & $F$ & & R & & $R$ & \begin{tabular}{l|l}
$R$ & $R$ \\
\end{tabular} & & \begin{tabular}{|l|l|}
$R$ \\
\end{tabular} & $F$ & 18 & 10 & \\
\hline $4-2 . \quad 24-25$ & 21.94 & & 18 & $F \mid A$ & A & & $F \mid$ & $R$ & A & \begin{tabular}{l|l}
$F$ & $R$ \\
\end{tabular} & & \begin{tabular}{|l|l|}
$F$ & $F$ \\
\end{tabular} & $F$ & $R$ & & & R & & $R$ & $F$ & $F$ & & \begin{tabular}{l|l}
$R$ & $R$ \\
\end{tabular} & $R$ & $\mathrm{R}$ & $R$ & $R$ & $R$ & $F$ & 6 & 8. & \\
\hline $4-3,24-25$ & 23.44 & & \begin{tabular}{l|l}
$R$ & I
\end{tabular} & $F \mid A$ & \begin{tabular}{l|l}
$A$ & $R$ \\
\end{tabular} & & F P & $R$ & A & \begin{tabular}{l|l}
$R$ & $F$ \\
\end{tabular} & & \begin{tabular}{|l|l}
$F$ & $F$ \\
\end{tabular} & $F$ & $F$ & & & \begin{tabular}{l|l}
$R$ \\
$R$
\end{tabular} & & $\mathrm{~A}$ & $F$ & $\mathrm{~F}$ & & \begin{tabular}{l|l}
$R$ & $R$ \\
\end{tabular} & & $F$ & \begin{tabular}{l|l}
$R$ & $F$ \\
\end{tabular} & $R$ & $\begin{array}{ll}R & R \\
\end{array}$ & $F$ & 8 & 7.5 & \\
\hline 4-4. $24-25$ & 24.94 & & & $F A$ & A & & $F$ & $R$ & A & \begin{tabular}{l|l}
$R$ & $F$ \\
\end{tabular} & & \begin{tabular}{l|l}
$F$ & $F$ \\
\end{tabular} & $\mathrm{~F}$ & $F$ & & & R & $R$ & $\mathrm{R}$ & $F$ & $\mathrm{~F}$ & & $\begin{array}{ll}R & R \\
R\end{array}$ & $R$ & $F$ & R F & $\mathrm{R}$ & $\begin{array}{ll}R & R \\
\end{array}$ & $F$ & E & 8. & \\
\hline 4-5. $24-25$ & 26.44 & & & \begin{tabular}{c|c}
$\mathrm{F} A$ \\
\end{tabular} & A & & $F / F$ & $R$ & A & \begin{tabular}{l|l}
$F$ & $F$ \\
\end{tabular} & & \begin{tabular}{|l|l}
$F$ & $F$ \\
\end{tabular} & $\mathrm{~F}$ & $F$ & & & R & & $R$ & $F$ & $\mathrm{~F}$ & & \begin{tabular}{l|l}
$R$ & $R$ \\
\end{tabular} & & $\mathrm{R}$ & \begin{tabular}{l|l}
$F R$ \\
\end{tabular} & $R$ & \begin{tabular}{l|l}
$R$ \\
\end{tabular} & $F$ & 6 & 12. & \\
\hline 4. CC & 28. 30 & & & $R A$ & \begin{tabular}{l|l}
$A$ & $R$ \\
\end{tabular} & & F & $R$ & A & \begin{tabular}{l|l}
$R$ & $F$ \\
\end{tabular} & & $F$ & $F$ & $R$ & & & & $\mathrm{R} / \mathrm{R}$ & \begin{tabular}{l|l|}
$R$ & $R$ \\
\end{tabular} & $F$ & $F$ & & \begin{tabular}{l|l}
$R$ & $R$ \\
\end{tabular} & $R$ & $F$ & \begin{tabular}{l|l}
$R$ & $F$ \\
\end{tabular} & $R$ & \begin{tabular}{l|l}
$R$ & $R$ \\
\end{tabular} & $F$ & $R$ & 15. & \\
\hline $5-1.24-25$ & 30. 15 & & & \begin{tabular}{l|l}
$R$ & $A$
\end{tabular} & A & & $F \mid F$ & $R$ & A & \begin{tabular}{l|l}
$R$ & $R$ \\
\end{tabular} & & \begin{tabular}{|l|l|l|}
$R$ & \\
\end{tabular} & $F$ & $R$ & & & \begin{tabular}{l|l}
$R$ & \\
\end{tabular} & & $\mathrm{R}$ & $F$ & $F$ & & \begin{tabular}{l|l}
$R$ & $R$ \\
\end{tabular} & & $F$ & \begin{tabular}{l|l}
$R$ & $F$ \\
\end{tabular} & & \begin{tabular}{l|l}
$R$ & $R$ \\
\end{tabular} & $F$ & A & 10. & \\
\hline 5-2. $24-25$ & 31.65 & & & $R A$ & \begin{tabular}{l|l}
$A$ & $R$ \\
\end{tabular} & & $F \mid F$ & $R$ & A & \begin{tabular}{l|l}
$F$ & $F$ \\
\end{tabular} & & \begin{tabular}{l|l|l|}
$F$ & $F$ \\
\end{tabular} & $F$ & $\mathrm{R}$ & & & $R$ & & $R$ & $F$ & $F$ & & $\begin{array}{ll}R & R \\
\end{array}$ & $R$ & $R$ & \begin{tabular}{l|l}
$R$ & $R$ \\
\end{tabular} & $R$ & \begin{tabular}{l|l}
$R$ & $R$ \\
\end{tabular} & $F$ & ta & 10. & \\
\hline $5-3,24-25$ & 33. 15 & & & F & $\mathrm{F}$ & & $R$ & & $F$ & \begin{tabular}{l|l}
$R$ & \\
\end{tabular} & & $\begin{array}{lll}R & F \\
\end{array}$ & $R$ & & & & & & $R$ & $F$ & $R$ & & & & $\mathrm{R}$ & & $R$ & $R$ & $R$ & 1 & .003 & \\
\hline $5-4,24-25$ & 34.65 & & & \begin{tabular}{l|l}
$R$ & $F$ \\
\end{tabular} & $\mathrm{~F}$ & & & $R$ & $F$ & $R$ & & $R$ & R & 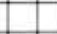 & & & & & $R$ & $F$ & $R$ & & $R$ & & $R$ & $\mathrm{~F}$ & & & $R$ & 1 & 02 & \\
\hline $5-5,24-25$ & 36. 15 & & & $\begin{array}{lll}R & F \\
\end{array}$ & $F \quad R$ & & $\mathrm{~F}$ & & A & \begin{tabular}{l|l}
$R$ & $R$ \\
\end{tabular} & & \begin{tabular}{l|l}
$R$ & $F$ \\
\end{tabular} & $R$ & $R$ & & & & & $R$ & & $R$ & & $R$ & & $R$ & $\begin{array}{ll}R & F \\
\end{array}$ & & \begin{tabular}{l|l} 
& $R$ \\
\end{tabular} & $R$ & t & 3. 2 & \\
\hline 5. CC & 36.73 & & & \begin{tabular}{l|l}
$R$ & $F$ \\
\end{tabular} & $\mathrm{~F}$ & & \begin{tabular}{l|l|l|}
$F$ & \\
\end{tabular} & $R$ & A & $\begin{array}{ll}R & R \\
\end{array}$ & & \begin{tabular}{l|l}
$F$ & $F$ \\
\end{tabular} & $\mathrm{~F}$ & $R$ & & & & $R$ & $R$ & $F$ & $\mathrm{~F}$ & & \begin{tabular}{l|l}
$R$ & $R$ \\
\end{tabular} & $\mathrm{R}$ & $\mathrm{R}$ & \begin{tabular}{l|l}
$R$ & $F$ \\
\end{tabular} & $R$ & \begin{tabular}{l|l}
$R$ & $R$ \\
\end{tabular} & $F$ & I & 10. & \\
\hline $6-1.24-25$ & 39. 85 & & & 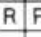 & $F$ & & \begin{tabular}{l|l}
$R$ & $F$
\end{tabular} & $\mathrm{R}$ & c & $\begin{array}{ll}R & R \\
\end{array}$ & & \begin{tabular}{l|l}
$R$ & $P$ \\
\end{tabular} & R. & $R$ & & & & $R$ & $R$ & $F$ & $R$ & & \begin{tabular}{l|l}
$R$ \\
\end{tabular} & $\mathrm{R}$ & $\mathrm{R}$ & $\begin{array}{lll}R & F \\
\end{array}$ & $R$ & \begin{tabular}{l|l}
$R$ & $R$ \\
\end{tabular} & $R$ & fa & 2.5 & \\
\hline $6-2.24-25$ & 41,35 & & & & & & & & $F$ & $R$ & & F & $R$ & & & & & & & & & & & & & & & \begin{tabular}{l|l} 
\\
$R$
\end{tabular} & $R$ & c & 1. & \\
\hline $6-4, \quad 24-25$ & 44. 35 & & & F & R & & $\mathrm{R}$ & & $F$ & \begin{tabular}{l|l}
$R$ \\
\end{tabular} & & & & $R$ & & & & $R$ & 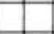 & & $R$ & & & & & $\mathrm{R}$ & $R$ & & $R$ & $A$ & 2. & \\
\hline 6. $c c$ & 37. 82 & & & $\begin{array}{ll}R & F \\
\end{array}$ & $\begin{array}{ll}\mathrm{F} \\
\mathrm{R}\end{array}$ & & $\mathrm{R}$ & & A & $\begin{array}{ll}R & R \\
\end{array}$ & & $F$ F & $\mathrm{R}$ & $R$ & & & R & $R$ & $R$ & $F$ & $\mathrm{~F}$ & & \begin{tabular}{l|l}
$R$ & $R$ \\
\end{tabular} & & $\mathrm{R}$ & $R F$ & $R$ & R & $F$ & $A$ & 48. & \\
\hline $7-1,24-25$ & 49. 55 & & & $\begin{array}{ll}R & F \\
\end{array}$ & F & & $\mathrm{F}$ & & A & $\begin{array}{ll}R & R \\
\end{array}$ & & \begin{tabular}{|l|l}
$F$ & $F$ \\
\end{tabular} & R & $R$ & & & $\mathrm{R}$ & $R$ & $R$ & $F$ & $R$ & & \begin{tabular}{l|l}
$\mathrm{R}$ & $\mathrm{R}$ \\
\end{tabular} & $R$ & & $R F$ & $R$ & \begin{tabular}{l|l}
$R$ & $R$ \\
\end{tabular} & $F$ & A & 10. & \\
\hline $7-3,24-25$ & 52.55 & & & $F$ & F & & R F & $\mathrm{R}$ & A & $\begin{array}{ll}R & F \\
\end{array}$ & & A F F & $\mathrm{R}$ & & & & & & $R$ & $\mathrm{R}$ & $R$ & & $R$ & & & $\begin{array}{lll}R & F \\
\end{array}$ & & \begin{tabular}{l|l}
$R$ \\
\end{tabular} & $\mathrm{~A}$ & c & 4. & \\
\hline $7-4 \cdot 24-25$ & 54.05 & & & F & $R$ & & $R$ & & c & \begin{tabular}{l|l}
$R$ & $R$ \\
\end{tabular} & & $\begin{array}{lll}R & F \\
\end{array}$ & $R$ & H & & & & & $R$ & $\mathrm{R}$ & $R$ & & & & R & $R$ & $R$ & & $R$ & A & 6.6 & \\
\hline $7 . \mathrm{cc}$ & 57.07 & & & \begin{tabular}{l|l}
$R$ & $F$ \\
\end{tabular} & F & & R F & $R$ & $F$ & \begin{tabular}{l|l}
$R$ & $R$ \\
\end{tabular} & & $\begin{array}{lll}A & F \\
\end{array}$ & R & $R$ & & & & & $R$ & $F$ & $R$ & & $R$ & $R$ & $R$ & \begin{tabular}{l|l}
$R$ & $F$ \\
\end{tabular} & & $R$ & $R$ & \begin{tabular}{l|l}
$R$ & $A$ \\
\end{tabular} & 20. & \\
\hline $8-1.24-25$ & 59.25 & & & $\begin{array}{ll}R & F \\
\end{array}$ & $\begin{array}{l}\mathrm{F} \\
\end{array}$ & & $F$ & $R$ & A & \begin{tabular}{l|l}
$F$ & $R$ \\
\end{tabular} & & $R F$ & $R$ & $R$ & & & & & $R$ & $\mathrm{R}$ & $R$ & & $R$ & & & \begin{tabular}{l|l}
$R$ & $F$ \\
\end{tabular} & $R$ & \begin{tabular}{l|l}
$R$ \\
\end{tabular} & $R$ & A & 20. & \\
\hline $8-2.24-25$ & 60.75 & & & 10 & \begin{tabular}{c|c}
$C$ & $R$ \\
\end{tabular} & & $F$ & $\mathrm{~F}$ & A & \begin{tabular}{l|l}
$R$ & $R$ \\
\end{tabular} & & \begin{tabular}{l|l}
$R$ & $F$ \\
\end{tabular} & $\mathrm{~F}$ & $R$ & & & & $R$ & $R$ & $F$ & R & & R & & $\mathrm{R}$ & $\mathrm{R}$ & $R$ & $\mathrm{R}$ & $R$ & R $A$ & 16.6 & \\
\hline 8-4. $24-25$ & 63. 75 & & & A & A & & $F$ & $\mathrm{R}$ & A & \begin{tabular}{l|l}
$F$ & $R$ \\
\end{tabular} & & \begin{tabular}{l|l}
$R$ & $F$ \\
\end{tabular} & $\mathrm{~F}$ & $R$ & & & \begin{tabular}{l|l}
$R$ \\
\end{tabular} & $R$ & $R$ & $F$ & $F$ & & \begin{tabular}{l|l}
$R$ & $R$ \\
\end{tabular} & & $\mathrm{R}$ & $\mathrm{F}$ & $R$ & $R$ & $F$ & $R A$ & 20. & \\
\hline $8 . \mathrm{CC}$ & 65,34 & & & $\begin{array}{ll}R & A \\
\end{array}$ & A & & \begin{tabular}{l|l} 
C \\
\end{tabular} & \begin{tabular}{l|l|}
$R$ & $R$ \\
\end{tabular} & A & \begin{tabular}{l|l}
$R$ & $R$ \\
\end{tabular} & & $R$ R F & R & $R$ & & $R$ & $R$ & & \begin{tabular}{l|l|}
$R$ & $R$ \\
\end{tabular} & $F$ & $R$ & & R & & $R$ & $\mathrm{~F}$ & $R$ & \begin{tabular}{l|l}
$R$ & $R$ \\
\end{tabular} & $R$ & $A$ & 16. 6 & \\
\hline $9-1,24-25$ & 68.95 & & & A & A & & $\mathrm{F} / \mathrm{F}$ & \begin{tabular}{l|l}
$R$ & \\
\end{tabular} & A & \begin{tabular}{l|l}
$R$ & $F$ \\
\end{tabular} & & R F & $\mathrm{F}$ & $\mathrm{R}$ & & & $R$ & & $R$ & $F$ & $R$ & & \begin{tabular}{l|l}
$R$ & $R$ \\
\end{tabular} & & $\mathrm{R}$ & $R$ & & R & $R$ & A & 13.3 & \\
\hline $9-3.24-25$ & 71.95 & & & A & A & & $F$ & & A & $\begin{array}{ll}R & R \\
\end{array}$ & & $R F$ & $R$ & $R$ & & & $R$ & & $R$ & $F$ & $R$ & & $\mathrm{R}$ & & $\mathrm{R}$ & $R$ & & & & c & 9. & \\
\hline 9. cc & 73. 20 & & & c & c & & & $\mathrm{R}$ & c & $R$ & & $R F$ & & & & & & & & $\mathrm{R}$ & $R$ & & $\mathrm{R}$ & R & & $\begin{array}{l} \\
\end{array}$ & & & 1 & A & $\rightarrow 1$ & \\
\hline $10-1,24-25$ & 78.65 & & & c & c & & $F$ & $R$ & c & $R$ & & \begin{tabular}{l|l}
$R$ & $F$ \\
\end{tabular} & $R$ & $\mathrm{R}$ & & & R & & $\mathrm{R}$ & $\mathrm{R}$ & $R$ & & $R$ & & & \begin{tabular}{l|l}
$R$ & $R$ \\
\end{tabular} & $R$ & $R$ & $A$ & $\begin{array}{lll}R & C \\
\end{array}$ & 8. & \\
\hline $10-2, \quad 24-25$ & 80. 15 & & & c & c & & $F$ & $R$ & c & $\mathrm{A}$ & & R F F & & $R$ & & & \begin{tabular}{l|l}
$R$ & \\
\end{tabular} & & $\mathrm{R}$ & $\mathrm{A}$ & $R$ & & $\mathrm{R}$ & & $R$ & & $\mathrm{R}$ & $R$ & $R$ & TA & 2. & \\
\hline $10 . c c$ & 84.50 & & & $\begin{array}{lll}R & C \\
\end{array}$ & c & & $R$ & $R$ & A & \begin{tabular}{l|l}
$R$ & $R$ \\
\end{tabular} & & \begin{tabular}{l|l}
$R$ & $F$ \\
\end{tabular} & $R$ & & & & & & & R & R & & $R$ & & $R$ & $R$ & $t$ & $\mathrm{R}$ & $R$ & A & 2.5 & \\
\hline $11-1,24-25$ & 88.25 & & & $R \mid C$ & c) & & $F \mid F$ & $\mathrm{~F}$ & A & \begin{tabular}{l|l}
$R$ & $F$ \\
\end{tabular} & & F F & $R$ & $R$ & & & & & $\mathrm{R}$ & $F$ & $F$ & & $R$ & & $R$ & $\mathrm{R}$ & $R$ & \begin{tabular}{l|l}
$R$ \\
\end{tabular} & $R$ & c & 10. & \\
\hline $11-2 \cdot 24-25$ & 89.75 & & & \begin{tabular}{l|l}
$R$ & $F$ \\
\end{tabular} & & & \begin{tabular}{|l|l|} 
\\
\end{tabular} & & $F$ & \begin{tabular}{l|l}
$R$ & $R$ \\
\end{tabular} & & $R$ & & $R$ & & & & & & & $R$ & & & & & $R$ & & & & F & 2. & \\
\hline $11-3,24-25$ & 91.25 & & & & & & \begin{tabular}{l|l}
$R$ & $F$ \\
\end{tabular} & $R$ & $F$ & $R$ & & $R$ & & & & & & & & $\mathrm{R}$ & $R$ & & $R$ & & & $R$ & & 1 & 1 & 10 & .72 & \\
\hline $11 . c c$ & 93,04 & & & $\begin{array}{ll}R \\
R\end{array}$ & c & & F F F & $R$ & A & $\begin{array}{lll}\mathrm{F} & \mathrm{C} \\
\end{array}$ & & $F \mid F$ & $\mathrm{~F}$ & $R$ & & & $R$ & $R$ & $R$ & $F$ & $F$ & & $R$ & $R$ & $R$ & $R$ & $R$ & \begin{tabular}{l|l}
$R$ & $R$ \\
\end{tabular} & $F$ & $R \mid C$ & 33.3 & \\
\hline $12-1, \quad 24-25$ & 97.85 & $\mathrm{R}$ & & $\begin{array}{lll} & C \\
\end{array}$ & c & & \begin{tabular}{l|l}
$F$ & $F$ \\
\end{tabular} & $\mathrm{~F}$ & A & \begin{tabular}{l|l}
$F$ & $C$ \\
\end{tabular} & & $F$ & $R$ & $R$ & & $\mathrm{R}$ & & $R$ & $\mathrm{R}$ & $F$ & $F$ & & $\mathrm{R}$ & $R$ & $F$ & $R$ & & \begin{tabular}{l|l}
$R$ & $R$ \\
\end{tabular} & $F$ & $1 c$ & 40. & \\
\hline $12-3,24-25$ & 100.85 & & & $\mathrm{~F} C \mathrm{C}$ & c & & $F \mid F$ & $\mathrm{~F}$ & A & \begin{tabular}{l|l}
$F$ & $C$ \\
\end{tabular} & & $F$ F & $F$ & $R$ & & & & $R$ & $\mathrm{R}$ & $F$ & $F$ & & $\mathrm{R}$ & $\mathrm{R}$ & $R$ & $\mathrm{~F}$ & $R$ & \begin{tabular}{l|l}
$R$ & $R$ \\
\end{tabular} & $F$ & s & 31.25 & \\
\hline $12 . \mathrm{cc}$ & 102.10 & & t & $R \mid C$ & c & & $F \mid F$ & $\mathrm{~F} \mid \mathrm{R}$ & A & $\begin{array}{ll}\mathrm{F} & \mathrm{C} \\
\end{array}$ & & $F$ & $R$ & $F$ & & & & & $R$ & F & $F$ & & $\begin{array}{ll}R \\
\end{array}$ & & & \begin{tabular}{l|l}
$R$ & $R$ \\
\end{tabular} & $R$ & \begin{tabular}{l|l}
$F$ & $R$ \\
\end{tabular} & $F$ & te & 37.5 & \\
\hline $13-1.24-25$ & 107.45 & & & $R \mid C$ & c & & \begin{tabular}{|l|l|l|}
$F$ & $F$ \\
\end{tabular} & $\mathrm{~F}$ & v & $\begin{array}{lll}R & C \\
\end{array}$ & & \begin{tabular}{l|l}
$F$ & $F$ \\
\end{tabular} & $R$ & $F$ & & & & & $\mathrm{R}$ & $F$ & $\mathrm{~F}$ & $\mathrm{R}$ & $R$ & & F & $F \mid R$ & & \begin{tabular}{l|l}
$R$ & $R$ \\
\end{tabular} & $F$ & \begin{tabular}{l|l}
$R$ & $A$ \\
$R$
\end{tabular} & 40. & \\
\hline $13-2, \quad 24-25$ & 108.95 & & & $\begin{array}{lll}R & C \\
\end{array}$ & C & & \begin{tabular}{|l|l}
$F$ & \\
\end{tabular} & \begin{tabular}{l|l|}
$F$ & $R$ \\
\end{tabular} & vif & $\begin{array}{ll}F & C \\
\end{array}$ & & $\mathrm{~F} / \mathrm{F}$ & $R$ & $F$ & & & & $R$ & & $F$ & $\begin{array}{ll}F & R \\
\end{array}$ & $\mathrm{R}$ & $R$ & & $R$ & $\mathrm{~F}$ & & $F \mid R$ & $F$ & $R \mid C$ & 60. & \\
\hline 13-3. $24-25$ & 110.45 & & & $\begin{array}{lll}R & C \\
\end{array}$ & c & & $F$ & $\begin{array}{ll}F & R \\
\end{array}$ & A & \begin{tabular}{l|l}
$R$ & $C$ \\
\end{tabular} & & \begin{tabular}{l|l}
$F$ & $F$ \\
\end{tabular} & $R$ & $F$ & & & & & & $F$ & $F$ & & $R$ & & $R$ & $\begin{array}{ll}R & R \\
\end{array}$ & & $\begin{array}{ll}R & R \\
\end{array}$ & $F$ & R & 62.5 & \\
\hline $13-4, \quad 24-25$ & 111.95 & & & $\mathrm{~F} / \mathrm{C}$ & C & & \begin{tabular}{|l|l|l|}
$F$ & $F$ \\
\end{tabular} & \begin{tabular}{l|l|}
$F$ & $R$ \\
\end{tabular} & v & $\begin{array}{lll}R & C \\
\end{array}$ & & $F$ & $R$ & $\mathrm{~F}$ & & & & $R$ & $R$ & $F$ & $\mathrm{~F}$ & $\mathrm{R}$ & $R$ & & $\mathrm{R}$ & \begin{tabular}{l|l}
$R$ & $R$ \\
\end{tabular} & & $\mathrm{~F} / \mathrm{R}$ & $F$ & $\mathrm{R} / \mathrm{A}$ & 50. & \\
\hline $13 . \mathrm{CC}$ & 113.61 & & & $\mathrm{R} \mid \mathrm{C}$ & c & & $F$ & \begin{tabular}{l|l|}
$F$ & $R$ \\
\end{tabular} & A & $\begin{array}{rl}R & C \\
\end{array}$ & & \begin{tabular}{|l|l|l|}
$F$ & $F$ \\
\end{tabular} & R & $F$ & & & & & $R$ & $F$ & $\mathrm{~F}$ & & \begin{tabular}{l|l}
$R$ & $R$ \\
\end{tabular} & $R$ & $\mathrm{R}$ & $\mathrm{F} / \mathrm{R}$ & $R$ & $F \mid R$ & $F$ & $R / \mathrm{G}$ & 12. & \\
\hline $14-1,24-25$ & 117.05 & & & $R / F$ & $\mathrm{~F}$ & & $\mathrm{~F} / \mathrm{F}$ & $\mathrm{F}$ & A & \begin{tabular}{l|l}
$R$ & $F$ \\
\end{tabular} & & \begin{tabular}{l|l|l}
$F$ & $F$ \\
\end{tabular} & R & $R$ & & $\mathrm{~F}$ & $R$ & & R & $F$ & $F$ & & R F & $R$ & $F$ & $\mathrm{~F} / \mathrm{F}$ & $R$ & $\begin{array}{l}R \\
R\end{array}$ & $R$ & tc & 12. 5 & \\
\hline $14-2, \quad 24-25$ & 118.45 & & & \begin{tabular}{l|l}
$R$ & $F$ \\
\end{tabular} & $F$ & & $\mathrm{R} F \mathrm{~F}$ & $\mathrm{R}$ & A & \begin{tabular}{l|l}
$R$ & $F$
\end{tabular} & & R & $\mathrm{R}$ & $R$ & & & & & $R$ & $R$ & $\mathrm{~F}$ & & $R$ & & R & \begin{tabular}{l|l}
$R$ & $R$ \\
\end{tabular} & & \begin{tabular}{l|l}
$R$ & $R$ \\
\end{tabular} & $R$ & s & 6. & \\
\hline $14 . C C$ & 119.17 & & & & & & & & C P & $F \mid R$ & & R F F & $R$ & & & & & & & & $\mathrm{R}$ & & & & R & $\mathrm{R}$ & & & & c & 1. & \\
\hline $15-1,24-25$ & 126.65 & & R F & $\mathrm{R} C$ & C & & $\mathrm{F} F$ & $\mathrm{~F}$ & A & $F / F$ & & $F F$ & & $\mathrm{R}$ & & & & $R$ & $R$ & $F$ & c & & \begin{tabular}{l|l}
$R$ & $F$ \\
\end{tabular} & $R$ & $F$ & $\mathrm{~F}[\mathrm{R}$ & $R$ & $F \mid R$ & $\mathrm{~F}$ & c & 375. & \\
\hline $15-2 \cdot \quad 24-25$ & 128.15 & & R F & $R C$ & c. & & \begin{tabular}{l|l}
$F$ & $F$ \\
\end{tabular} & $F R$ & VFf & \begin{tabular}{ll|}
$F$ & $C$ \\
\end{tabular} & & $F F$ & $\mathrm{~F}$ & \begin{tabular}{l|l}
$F$ & $R$ \\
\end{tabular} & R. & $R$ & & & $\mathrm{R}$ & c| & c & & \begin{tabular}{l|l}
$R$ & $F$ \\
\end{tabular} & $\mathrm{~F}$ & $\mathrm{~F}$ & $F \mid F$ & $R$ & $F \mid R$ & c & R C & 200. & \\
\hline $15-3 . \quad 24-25$ & 129.65 & & \begin{tabular}{l|l}
$R$ & $R$ \\
\end{tabular} & & & & & $F R$ & V P F & & & & & & & & & $R$ & & & & & & & $F$ & & & & & & 1250. & \\
\hline
\end{tabular}


Table 11 (continued).

\begin{tabular}{|c|c|c|c|c|c|c|c|c|c|c|c|c|c|c|c|c|c|c|c|c|c|c|c|c|c|c|c|c|c|c|c|c|c|c|c|c|c|}
\hline $\begin{array}{l}\text { Core- } \\
\text { Section } \\
\text { (interval } \\
\text { in } \mathrm{cm} \text { ) }\end{array}$ & $\begin{array}{c}\text { Sub- } \\
\text { bottom } \\
\text { depth } \\
(\mathrm{m})\end{array}$ & 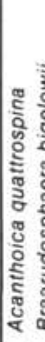 & & & & & & & & & & & & & & & & & & & & & & & & $\begin{array}{l}\text { के } \\
\text { के } \\
\text { के }\end{array}$ & 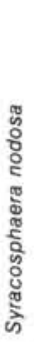 & & 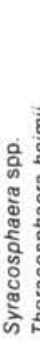 & & : & & & & & 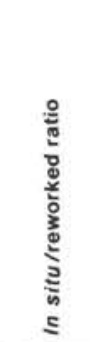 & $\begin{array}{l}\text { Zone or } \\
\text { subzone }\end{array}$ \\
\hline $15 . \mathrm{CC}$ & 130.46 & & $\mathrm{R}$ & C & $\mathrm{R}$ & & $\bar{F}$ IF & F & $\mathrm{v}$ & $F$ & $\mathrm{~F}$ & $F$ & $F$ & If & $R$ & & $\mathrm{R} F$ & R & $R$ & & & $\bar{F}$ & C & & $\mathrm{F}$ & $F$ & $\mathrm{R}$ & $F \mid$ & $\bar{F} F$ & $R$ & $\mathrm{~F}$ & $\mathrm{~F}$ & $F$ & $R$ & & 300. & \multirow{4}{*}{$\begin{array}{c}\text { Emiliania } \\
\text { huxleyi } \\
\text { acme }\end{array}$} \\
\hline $16-1, \quad 24-25$ & 136.25 & & & $F$ & & & F F r & R & $\mathrm{F}$ & R & & $F$ & $R$ & & & & & & & & & $R$ & $R$ & & & & & $R$ & $R$ & & & & $R$ & $F$ & & 25. & \\
\hline $16-2, \quad 24-25$ & 137.25 & & $\mathrm{R}$ & C & $R$ & & $\mathrm{FFF}$ & F & A & \begin{tabular}{l|l}
$c$ & \\
\end{tabular} & E & C & $F$ & R & & & & \begin{tabular}{l|l}
$R$ & $F$ \\
\end{tabular} & & $R$ & & $F$ & $F$ & & $\mathrm{R}$ & $R$ & & $F$ & $\mathrm{~F}$ & $R$ & $R$ & R & $F$ & $R$ & & 750. & \\
\hline $16-4, \quad 24-25$ & 140.75 & \begin{tabular}{l|l}
$R$ & $F$ \\
\end{tabular} & $\mathrm{R}$ & $\mathrm{C}$ & $\mathrm{R}$ & & $F$ & f & $\mathrm{v}$ & $\begin{array}{ll}\text { C } \\
\end{array}$ & A & C & $F$ & ti & & & $R$ & $F$ & & & & c & c & R & $\mathrm{R}$ & $\mathrm{F}$ & $R$ & c & $R F$ & $=$ & $\mathrm{R}$ & $\mathrm{F}$ & $F$ & $\mathrm{R}$ & & 3000. & \\
\hline $16 . \mathrm{CC}$ & 141.74 & $\begin{array}{ll}R & F \\
\end{array}$ & $2 \pi$ & C & $R$ & & c/f & $F$ & A & A & $\sqrt{R}$ & A & $F$ & ti & & & $t_{F}$ & R & & & R & c & C & & $\mathrm{F}$ & $F$ & $R$ & \begin{tabular}{l|l} 
C & \\
\end{tabular} & $\begin{array}{l}R \\
R\end{array}$ & R & $R$ & $\bar{R}$ & $F$ & & & 250. & \multirow{19}{*}{$\begin{array}{c}\text { Emiliania } \\
\text { huxleyi }\end{array}$} \\
\hline $17-1.30-31$ & 146.01 & $F_{F}$ & $F$ & C & $\mathrm{R}$ & $R$ & c] & Ef & A & A & & v & $F$ & ti & & $R$ & & $\begin{array}{l}R \\
R\end{array}$ & R & $\mathrm{R}$ & $R$ & c & C & & $F$ & $F$ & $F$ & F F & $\begin{array}{ll}R & F \\
\end{array}$ & R $F$ & $F$ & $\mathrm{~F}$ & $F$ & $R$ & & 900 & \\
\hline $17-2, \quad 30-31$ & 147.51 & $F$ & 2. $\mathrm{F}$ & C & $\mathrm{R}$ & $R$ & c) & F & $A$ & A & & v & $\mathrm{F}$ & & र $\mathrm{R}$ & & & F & & & & c & C & & $F$ & $R$ & $R$ & \begin{tabular}{|l|l}
$F$ \\
\end{tabular} & R F & F & $F$ & $\mathrm{~F}$ & c & $\mathrm{F}$ & & 400. & \\
\hline $17-3,30-31$ & 149.01 & & $F$ & C & 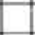 & & F F & Ff & C & \begin{tabular}{l|l} 
C \\
\end{tabular} & F & $\mathrm{v}$ & $F$ & ti & र $\mathrm{A}$ & & & $F$ & & & & c & C & & $F$ & $\mathrm{~F}$ & $R$ & $F$ & R F & $\mathrm{F}$ & $F$ & $\mathrm{~F}$ & $F$ & R & & 300. & \\
\hline $17 . c c$ & 150.71 & & $R$ & C & $R$ & & Fif & Fif & C & A & & v & $F$ & ti & & & & R F & & & & $\mathrm{F}$ & c & & $\mathrm{F}$ & $F$ & $R$ & $F$ & F & ? & $F$ & $\mathrm{~F}$ & $\mathrm{~F}$ & & & 100. & \\
\hline $18 . \mathrm{CC}$ & 158.40 & & & $F$ & & $R$ & $\mathrm{~F} F \mathrm{~F}$ & a & c & \begin{tabular}{l|l} 
C \\
\end{tabular} & & A & $R$ & & R & & & & & & & $\mathrm{F}$ & $F$ & & & & & $F$ & $R$ & $\mathrm{R}$ & & $R$ & $R$ & & & 24. & \\
\hline $19-1 . \quad 36-39$ & 165.47 & & & $\mathrm{~F}$ & & $R$ & tif & R & $F$ & F & & $F$ & $R$ & & & & & & & & & $F$ & $R$ & & & & & $R$ & F & & & $R$ & $R$ & & & 2.5 & \\
\hline $19-3, \quad 36-39$ & 168.47 & & R & $F$ & & & & & $\mathrm{~F}$ & F & & $\mathrm{F}$ & $R$ & & & & & & & & & $\mathrm{R}$ & $R$ & & $\mathrm{R}$ & & & $\begin{array}{lll} \\
R\end{array}$ & \begin{tabular}{l|l}
$R$ & $F$ \\
\end{tabular} & & & & $R$ & & & 5. & \\
\hline 19. cc & 169.10 & & & $\mathrm{R}$ & & & f & $R$ & $R$ & Ot & & $\mathrm{R}$ & $R$ & & & & & & & & & $R$ & & & & & & & $R F$ & ? & & & $R$ & & & 1. & \\
\hline $20-2, \quad 36-37$ & 176.57 & & & $F$ & & & & & $F$ & F & & c & & & & & & & & & & $R$ & $R$ & & & & & 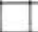 & & & & & R & $R$ & & 3. 3 & \\
\hline $20 . c c$ & 178.02 & & & $\mathrm{~F}$ & & & & & C & \begin{tabular}{|l|l}
$F$ \\
\end{tabular} & & c & $R$ & & & & & & & & & $R$ & $R$ & & & & & $\mathrm{R}$ & $\begin{array}{lll}R & F \\
\end{array}$ & & R & & $R$ & & c & 4. & \\
\hline $21 . c c$ & 184.30 & & $\mathrm{R}$ & C & $R$ & & $\mathrm{~F} F$ & & C & \begin{tabular}{l|l} 
\\
\end{tabular} & & $A$ & $F$ & & $=R$ & & & & & $\mathrm{R}$ & $R$ & c & $\mathrm{F}$ & & & $R$ & & $R$ & F F & & $R$ & $R$ & $F$ & $\mathrm{R}$ & & 24. & \\
\hline $22-1, \quad 39-40$ & 187.69 & F & R $\mathrm{R}$ & $A$ & $\mathrm{R}$ & & c) & $c$ & C & A & & A & $\mathrm{F}$ & ti & & & & f & R & & $R$ & C & $\mathrm{R}$ & $R$ & $R$ & R & & $F$ & $F \mid F$ & F & & R & c & $R$ & & 23. 7 & \\
\hline $22-2, \quad 30-31$ & 189.10 & $F$ & a $\mathrm{A}$ & $A$ & $R$ & & c) & c|f & C & A & & A & $F$ & ti & & & & F & F & & $R$ & c & C & & $\mathrm{R}$ & $R$ & $R$ & $F$ & $\mathrm{~F}$ & t & $R$ & $\mathrm{~F}$ & C & $\mathrm{R}$ & & 82.1 & \\
\hline $22-2 . \quad 61-62$ & 189.42 & F & a & A & $R$ & & c) & c) & C & A & & v & $F$ & tr & & & & & F & $R$ & $R$ & c & C & & R & $R$ & $R$ & $F$ & $\mathrm{~F}$ & F & $R$ & F & C & $R$ & & 90. & \\
\hline $22 . \mathrm{CC}$ & 189.75 & F & $2 \mathrm{R}$ & $A$ & & & $F$ & c. & C & A & & $\mathrm{v}$ & $F$ & & & & & & & $R$ & & c & $R$ & & $R$ & R & $\mathrm{R}$ & $F$ & Fi & F & R & $R$ & A & $R$ & & 78.2 & \\
\hline 23. cC & 203.72 & & $\mathrm{R}$ & $\mathrm{C}$ & & & $R F$ & R & $R$ & \begin{tabular}{l|l} 
c & ( \\
\end{tabular} & & C & $\mathrm{R}$ & & & & & & & & & $R$ & $R$ & & & & & $\mathrm{R}$ & Rf & & & & R & & $c$ & 2.4 & \\
\hline $24 . \mathrm{CC}$ & 206.72 & $\mathrm{~F}$ & a. & $F$ & & & $\mathrm{~F} F$ & $\mathrm{R}$ & $R$ & \begin{tabular}{l|l} 
c \\
\end{tabular} & & A & $R$ & & & & & & & & & $\mathrm{R}$ & $R$ & & & & $\mathrm{R}$ & $\mathrm{R}$ & $\mathrm{F}$ & & & & R & & A & 1.8 & \\
\hline $25, \mathrm{cc}$ & 208.70 & F & 2 & $F$ & & & Fif & R. & $R$ & $F$ & & c & $\mathrm{R}$ & & & & & & & & & $R$ & & & & $R$ & $\mathrm{R}$ & $\mathrm{R}$ & R & ] & & & $\mathrm{R}$ & & c] & 1.9 & \\
\hline
\end{tabular}

Note: For abundance, $\mathrm{H}=$ highly abundant, more than 100 specimens/field $\mathrm{V}=$ very abundant, $10-100$ specimens/field $\mathrm{A}=\mathrm{abundant} 1-10$ specimens/field $\mathrm{C}=$ common, 1 specimen $/ 2-10$ fields, $F=$ few. 1 specimen $/ 11-100$ fields, $R=$ rare, 1 specimen $/ 101-1000$ fields (when viewed at a magnification of $x 1250$ ).

the study of Holes E67-134 and E67-135 on the northwest Florida shelf by Chen (1978) and Gartner et al. (1983). These authors correlated the amount of detrital sedimentation (by means of percent carbonate) with global oxygen-isotope curves. They also noted an increase in the abundance of reworked nannofossils during the glacial intervals.

A similar correlation can be made between the in situ/ reworked ratio curve of Site 619 and the stable isotope curves of Emiliani (1972) and Anderson and Steinmetz $(1981,1983)$ (Fig. 6). The high peaks of this curve found in Core 619-1 (0-9 m sub-bottom) and Cores 619-15 through 619-18 (127-158 m sub-bottom) generally correlate with isotope Stages 1 and 5, respectively. The low values immediately below and above these peaks represent the cool Stages 2 and 4.

The in situ/reworked ratio curve of Site 619 can be related to the percentage curves of planktonic and benthic foraminifers (Kohl, this volume). In general, the higher percentages of warm planktonic foraminifers coincide with the higher values of the in situ/reworked ratio, indicating a relative decrease in the terrigenous component in the sediment during interglacial times.

The patterns of occurrences of reworked Cretaceous nannofossils differ from those of the reworked Cretaceous foraminifers. Whereas the Cretaceous foraminifers are restricted primarily to zones of coarser clastic deposition, the small reworked nannofossils can be abundant in any depositional facies with a fine textural component.

\section{CONCLUSIONS}

This preliminary study shows that the relative abundance of indigenous versus reworked taxa in transitional marine environments is a potential tool for both local and global correlations. More detailed quantitative investigation is needed to substantiate and refine these initial results:

1. The horizon marked by the first evolutionary occurrence of Emiliania huxleyi was not encountered at any of the Leg 96 sites.

2 . The datum level represented by the lowest stratigraphic occurrence of dominant $E$. huxleyi at Site 619 is dated by tephrochronology at 84,000 yr. ago.

3. The relative abundances of indigenous Quaternary taxa and reworked taxa is controlled by glacio-eustatic fluctuations during the late Pleistocene. This relationship can be documented by use of calculated in situ/reworked ratios.

4. Glacial coolings and interstadial warmings are responsible for the four-unit floral sequence observed in Leg 96 sediments. During cool glacial periods, large influxes of detrital, reworked Cretaceous nannofossils result in low in situ/reworked ratios. Reduced sedimentation rates during the warmer intervals yield a relative increase in the abundance of indigenous Quaternary taxa and higher in situ/reworked ratios.

5. The depositional facies has no significant effect on the pattern of occurrences of reworked Cretaceous 
Site 619

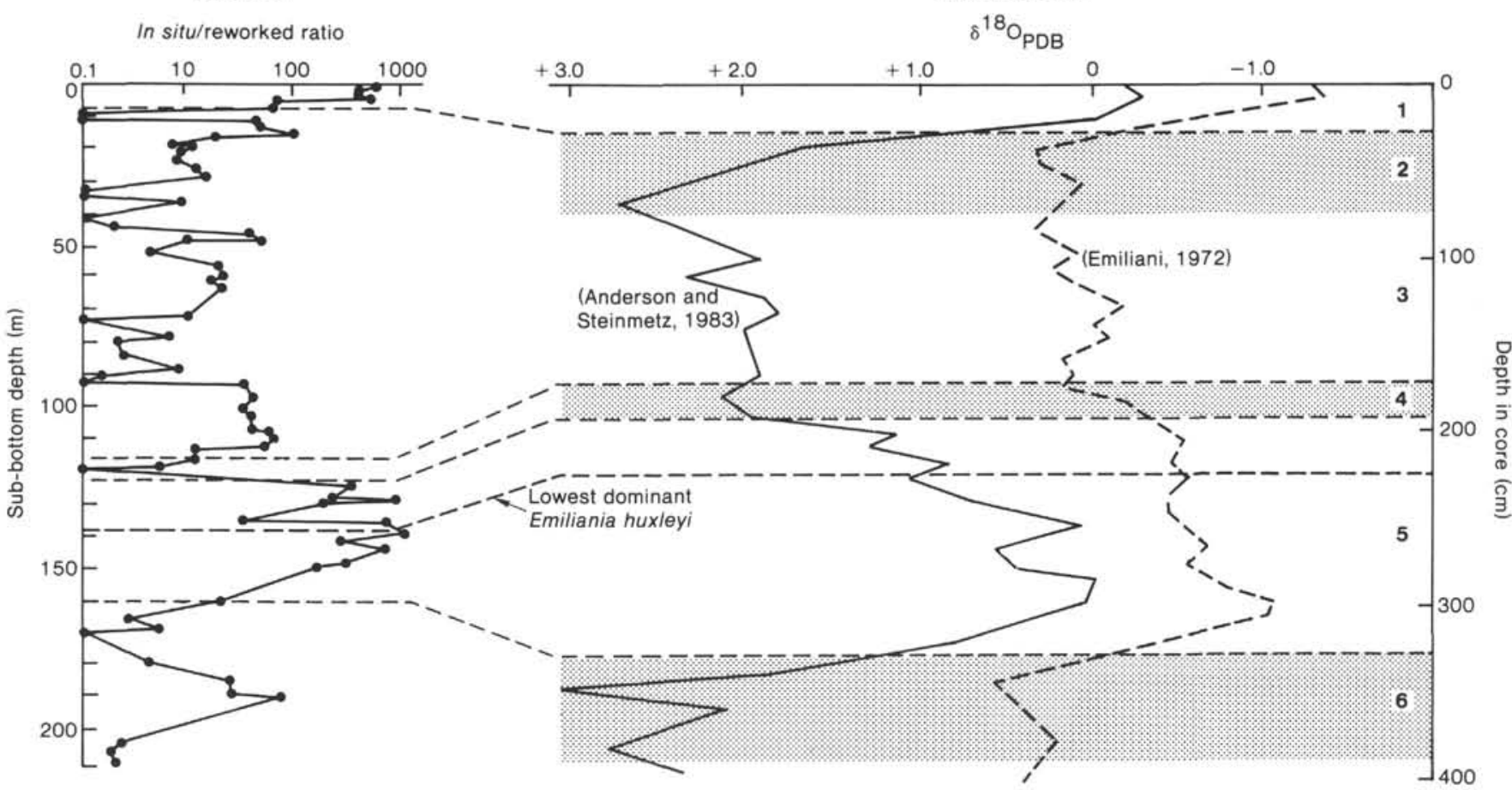

Figure 6. Comparison of the in situ/reworked ratio curve of Site 619 with stable isotope curves from the Caribbean Core P6304-4 (from Emiliani, 1972; Anderson and Steinmetz, 1983). Shaded areas represent the cool, even-numbered isotopic stages (shown on right-hand side of figure).

nannofossils. Reworked nannofossils can be abundant in any sediment with a fine textural component.

\section{REMARKS ON SELECTED CALCAREOUS NANNOFOSSIL TAXA}

Bibliographic references for these taxa are presented by Loeblich and Tappan (1966, 1968, 1969, 1970a, b, 1971, 1973), Heck (1979a, b, 1980 a, b, 1981a, b, 1982a, b) or by Steinmetz (1983a, b, 1984). Most of the calcareous nannofossil species considered in this study are documented elsewhere and need no further discussion. A broad, utilitarian species concept, however, is used for some of the taxa listed below.

Acanthoica quattrospina Lohmann, 1903. This taxon may be related to Rhabdosphaera stylifera Lohmann, 1902, which is classified with $R$. clavigera Murray and Blackman in this study.

Braarudosphaera bigelowii (Gran and Braarud) Deflandre, 1947.

Calyptrosphaera oblonga Lohmann, 1902, s. ampl., This taxon includes Calyptrosphaera catillifera (Kamptner) Gaarder, 1962 and may include poorly preserved specimens of Anthosphaera quatricornu (Schiller) Halldal and Markali, 1955. C. caltillifera is known only as a living species.

Ceratolithus cristatus Kamptner, 1950. Includes Ceratolithus telesmus Norris, 1965.

Crenalithus doronicoides (Black and Barnes) Roth, 1973, s. ampl. This taxon is used broadly and includes Coccolithus productus (Kamptner) Sachs and Skinner, 1973 and Crenalithus productellus Bukry, 1975. Many small coccoliths with no discernible bridge are included in $C$. doronicoides.

Coccolithus pelagicus (Wallich) Schiller, 1930.

Corisphaera gracilis Kamptner, 1937.

Cricolithus jonesii Cohen, 1965.

Cyclococcolithus leptoporus (Murray and Blackman) Kamptner, 1954.

Discosphaera tubifera (Murray and Blackman) Ostenfeld, 1900.

Emiliania huxleyi (Lohmann) Hay and Mohler, 1967.

Gephyrocapsa caribbeanica Boudreaux and Hay, 1967.

Gephyrocapsa oceanica Kamptner, 1943. G. omega Bukry, 1973 and G. lumina Bukry, 1973 were included in this taxon.

Gephyrocapsa protohuxleyi McIntyre, 1970. This taxon can only be identified with the scanning electron microscope.
Gephyrocapsa spp. (small). Among others, this grouping includes: $G$. aperta Kamptner, 1963; G. ericsonii McIntyre and Bé, 1967; G. kamptneri Deflandre and Fert, 1954; $G$. sinuosa Hay and Beaudry, 1973; and G. protohuxleyi McIntyre, 1970. With the light microscope $G$. protohuxleyi cannot be separated and is included with Gephyrocapsa spp. (small).

Hayaster perplexus (Bramlette and Riedel) Bukry, 1973.

Helicosphaera carteri (Wallich) Kamptner, 1954. Includes Helicopontosphaera kamptneri Hay and Mohler, 1967.

Helicosphaera hyalina Gaarder, 1970.

Helicosphaera wallichii (Lohmann) Okada and McIntyre, 1977.

Homozygosphaera schilleri (Kamptner) Okada and McIntyre, 1977. Same as Holodiscolithus macroporus (Deflandre) Roth, 1970.

Homozygosphaera wettsteinii (Kamptner) Halldal and Markali, 1955.

Neosphaera coccolithomorpha Lecal-Schlauder, 1950, s. ampl. This taxon includes Craspedolithus declivus (Kamptner) Nishida, 1970 and Cyclolithella annula McIntyre and Bé, 1967.

Oolithus fragilis (Lohmann) Okada and McIntyre, 1977.

Pontosphaera discopora Schiller, 1925 emed. Burns, 1973. This taxon includes forms with three rows of perforations.

Pontosphaera multipora (Kamptner), Roth, 1970. This taxon includes forms with two rows of perforations.

Pontosphaera scutellum Kamptner, 1952

Pontosphaera japonica (Takayma) Nishida, 1971.

Pontosphaera spp. Any species that cannot readily be placed in one of the four Pontosphaera species listed above is included in Pontosphaera spp.

Rhabdosphaera clavigera Murray and Blackman, 1898. Includes Rhabdosphaera stylifera Lohmann, 1902.

Scapholithus fossilis Deflandre, 1954.

Scyphosphaera apsteinii Lohmann, 1902.

Scyphosphaera pulcherrima Deflandre, 1942. Occurrences of this taxon may be reworked.

Scyphosphaera spp. Various forms of Scyphosphaera that do not fit the descriptions of $S$. apsteinii and $S$. pulcherrima are placed in this category. Many species of Scyphosphaera are described in the literature (Deflandre, 1942; Kamptner 1955).

Syracosphaera histrica Kamptner, 1941.

Syracosphaera lamina Lecal-Schlauder, 1951, s. ampl. This taxon includes species with longitudinal central bar: S. ribosa (Kamptner) 
Borsetti and Cati, 1972; S. lactaria (Lecal) Loeblich and Tappan, 1968; S. nana (Kamptner) Okada and McIntyre, 1977; S. ossa (Lecal) Loeblich and Tappan, 1968; S. tuberculata Kamptner, 1937; S. variabilis (Halldal and Markali) Okada and McIntyre, 1977.

Syracosphaera nodosa Kamptner, 1941.

Syracosphaera pulchra Lohmann, 1902.

Syracosphaera spp. Taxa not readily placed in the four Syracosphaera species listed above are placed in Syracosphaera spp.

Thoracosphaera heimii (Lohmann) Kamptner, 1941.

Thoracosphaera saxea Stradner, 1961.

Umbellosphaera irregularis Paasche, 1955.

Umbellosphaera tenuis (Kamptner), Paasche, 1955.

Umbilicosphaera mirabilis Lohmann, 1902. This taxon is not considered to be a junior synonym of Umbilicosphaera sibogae (WebervanBosse) Gartner, 1970. The original description and illustration of two concentric circles by Weber-vanBosse (1901) is not adequate to determine that $U$. mirabilis is equivalent to $U$. sibogae.

\section{Reworked Cenozoic Species}

Reworked Cenozoic calcareous nannofossils occur sporadically throughout the Leg 96 sites and include the following significant biostratigraphic taxa.

Cyclococcolithus tropicus (Kamptner) Gartner, Chen and Stanton, 1983. This may not be the same as C. macintyrei Bukry and Bramlette, 1969. Since the classification of these taxa is still questionable, we tabulated them together.

Discoaster asymmetricus Gartner, 1969.

Discoaster barbadiensis Tan, 1927.

Discoaster bollii Martini and Bramlette, 1963.

Discoaster berggrenii Bukry, 1971.

Discoaster brouweri Tan, 1927.

Discoaster deflandrei Bramlette and Riedel, 1954.

Discoaster pentaradiatus Tan, 1927.

Discoaster quinqueramus Gartner, 1969.

Discoaster surculus Martini and Bramlette, 1963.

Discoaster variabilis Martini and Bramlette, 1963.

Helicosphaera sellii (Bukry and Bramlette) Jafar and Martini, 1975.

Lithostromation perdurum Deflandre, 1942.

Pseudoemiliania lacunosa (Kamptner) Gartner, 1969

Reticulofenestra pseudoumbilica (Gartner) Gartner, 1969.

Sphenolithus abies Deflandre, 1954, s. ampl. This taxon also includes S. neoabies Bukry and Bramlette, 1969.

Sphenolithus heteromorphus Deflandre, 1953.

\section{Reworked Mesozoic Species}

Although Jurassic and Early Cretaceous species can be found, this assemblage is dominated by solution-resistant Late Cretaceous species. The most common of these are listed below.

Arkhangelskiella cymbiformis Vekshina, 1959.

Broinsonia parca (Stradner) Bukry, 1969.

Eiffellithus turriseiffeli (Deflandre and Fert) Reinhardt, 1965.

Lithastrinus floralis Stradner, 1962.

Micula decussata Vekshina, 1959.

Prediscosphaera cretacea (Arkhangelsky) Gartner, 1969.

Prediscosphaera spinosa (Bramlette and Martini) Gartner, 1968.

Watznaueria barnesae (Black) Perch-Nielsen, 1968.

\section{ACKNOWLEDGMENTS}

The authors would like to thank the following people: Drs. D. Bukry and S. W. Wise for reviewing this manuscript and offering many constructive comments; Dennis Greig, whose operation of the scanning electron microscope and knowledge of nannoplanktonology aided in the preparation of the plates; Bill Ventress and Chevron USA Inc., who provided the time and resources for this study; and Gisela Galjour for typewriting the manuscript.

\section{REFERENCES}

Anderson, T. F., and Steinmetz, J. C., 1981. Isotopic and biostratigraphic records of calcareous nannofossils in a Pleistocene core. Nature, 294 (5843):741-744.

1983. Stable isotopes in calcareous nannofossils; potential application to deep-sea paleoenvironmental reconstructions during the Quaternary. Utrecht Micropaleontol. Bull., 30:189-204.
Beard, J. H., Sangree, J. B., and Smith, L. A., 1982. Quaternary chronology, paleoclimate, depositional sequence, and eustatic cycles. Am. Assoc. Pet. Geol. Bull., 66:158-169.

Blow, W. H., 1969. Late middle Eocene to Recent planktonic foraminiferal biostratigraphy. In Brönniman, P., and Renz, H. H. (Eds.), Proc. Int. Conf. Planktonic Microfossils Ist.: Leiden (Brill), 6:199421.

Boudreaux, J. E., and Hay, W. W., 1967. Zonation of the latest Pliocene-Recent interval. Gulf Coast Assoc. Geol. Soc. Trans., 17:443445.

Bukry, D., 1973. Coccolith stratigraphy, Leg 10, DSDP In Worzel, J. L., Bryant W., et al., Init. Repts. DSDP, 10:Washington (U.S. Govt. Printing Office), 385-406.

1974. Coccoliths as paleosalinity indicators-Evidence from Black Sea. Am. Assoc. Pet. Geol. Mem., 20:353-363.

Bukry, D., and Bramlette, M. N., 1969. Coccolith age determination Leg 1, Deep Sea Drilling Project. In Ewing, M., Worzel, J. L., et al., Init. Repts. DSDP, 1:Washington (U.S. Govt. Printing Office), 369-387.

Chen, M. P., 1978. Calcareous nannoplankton biostratigraphy and paleoclimatic history of the Late Neogene sediments of the Northwest Florida Shelf [Ph.D. dissert.]. Texas A\&M University, College Station.

Ellis, C. H., and Lohman, W. H., 1979. Neogene calcareous nannoplankton biostratigraphy in eastern Mediterranean deep-sea sediments (DSDP Leg 42A, Sites 375 and 376). Mar. Micropaleontol., 4:61-84.

Ellis, C. H., Lohman, W. H., and Wray, J. L., 1972. Upper Cenozoic calcareous nannofossils from the Gulf of Mexico (Deep Sea Drilling Project, Leg 1, Site 3). Colo. Sch, Mines Quat., 67(3):1-103.

Emiliani, C., 1966. Paleotemperature analysis of Caribbean cores P6304-8 and P6304-9 and a generalized temperature curve for the past 425,000 years. J. Geol., 74(2):109-126.

1971. The last interglacial: Paleotemperatures and chronology. Science, 171:571-573.

1972. Quaternary paleotemperatures and the duration of the high-temperature intervals. Science, 178(4059):398-401.

Ericson, D. B., and Wollin, G., 1968. Pleistocene climates and chronology in deep-sea sediments. Science, 162:1227-1234.

Everett, R. W., 1982. Using nannofossil counts in the interpretation of subsurface deltas, Gulf Coast Assoc. Geol. Soc. Trans., 22:579-591.

Gartner, S., Chen, M. P., and Stanton, R. J., 1983. Late Neogene nannofossil biostratigraphy and paleoceanography of the northeastern Gulf of Mexico and adjacent areas. Mar. Micropaleontol., 8:17-50.

Gartner, S., and Emiliani, C., 1976. Nannofossil biostratigraphy and climatic stages of the Pleistocene. Am. Assoc. Pet. Geol. Bull., 60: $1562-1564$.

Hay, W. W., 1969. Preliminary dating by fossil calcareous nannoplankton, Deep Sea Drilling Project, Leg 1. In Ewing, M., Worzel, J. L., et al., Init. Repts. DSDP, 1:Washington (U.S. Govt. Printing Office), 388-391.

1970. Calcareous nannofossils from cores recovered on Leg 4. In Bader, R. G., Gerard, R. D., et al., Init. Repts. DSDP, 4: Washington (U.S. Govt. Printing Office), 455-501.

1973. Preliminary dating by fossil calcareous nannoplankton: In Worzel, J. L., Bryant, W., et al., Init. Repts. DSDP, 10: Washington (U.S. Govt. Printing Office), 375-383.

Heck, S. E., van, 1979a. Bibliography and taxa of calcareous nannoplankton. Int. Nannoplankton Assoc. Newsl., 1:AB I-V, A1-12, B1-27.

1979b. Bibliography and taxa of calcareous nannoplankton. Int. Nannoplankton Assoc. Newsl., 1:AB VI, A13-28, B2842.

1980a. Bibliography and taxa of calcareous nannoplankton. Int. Nannoplankton Assoc. Newsl., 2:5-34.

$1980 \mathrm{~b}$. Bibliography and taxa of calcareous nannoplankton. Int. Nannoplankton Assoc. Newsl., 2:43-81.

1981a. Bibliography and taxa of calcareous nannoplankton. Int. Nannoplankton Assoc. Newsl., 3:4-41.

1981b. Bibliography and taxa of calcareous nannoplankton. Int. Nannoplankton Assoc. Newsl., 3:51-86.

1982a. Bibliography and taxa of calcareous nannoplankton. Int. Nannoplankton Assoc. Newsl., 4:7-50. 
, 1982b. Bibliography and taxa of calcareous nannoplankton Int. Nannoplankton Assoc. Newsl., 4:65-96.

Kennett, J. P., and Huddleston, P., 1972. Late Pleistocene paleoclimatology, western Gulf of Mexico. Quat. Res., 2:38-69.

Ledbetter, M., 1984. Late Pleistocene tephrachronology in the Gulf of Mexico region. In Healy-Williams, N.(Ed.), Principles of Pleistocene Stratigraphy Applied to the Gulf of Mexico: Boston (IHRDC Press), pp. 119-148.

Loeblich, A. R., Jr., and Tappan, H., 1966. Annotated index and bibliography of the calcareous nannoplankton. Phycologia, 5:81-216.

1968. Annotated index and bibliography of the calcareous nannoplankton II. J. Paleontol., 42:584-598.

1969. Annotated index and bibliography of the calcareous nannoplankton III. J. Paleontol., 43:568-588.

1970a. Annotated index and bibliography of the calcareous nannoplankton IV. J. Paleontol., 44:558-574.

1970b. Annotated index and bibliography of the calcareous nannoplankton V. Phycologia, 9:157-174.

1971. Annotated index and bibliography of the calcareous nannoplankton VI. Phycologia, 10:315-339.

, 1973. Annotated index and bibliography of the calcareous nannoplankton VII. J. Paleontol., 47:715-759.

Martini, E., 1971. Standard Tertiary and Quaternary calcareous nannoplankton zonation. In Farinacci, A. (Ed), Proc. II Planktonic Conf. Roma: Rome (Edizioni Tecnoscienza), 2:739-785.

Okada, H., and Bukry, D., 1980. Supplementary modification and introduction of code numbers to the "Low-latitude coccolith biostratigraphic zonation" (Bukry, 1973; 1975). Mar. Micropaleontol., 5:321-325.

Pierce, R. W., and Hart, G. F., 1979. Phytoplankton of the Gulf of Mexico, taxonomy of calcareous nannoplankton. Geoscience and Man, 20:1-62.

Rögl, F., and Bolli, H. M., 1973. Holocene to Pleistocene planktonic foraminifera of Leg 15, Site 147 (Cariaco Basin (Trench), Caribbe- an Sea) and their climatic interpretation. In Edgar, N. T., Saunders, J. B., et al., Init. Repts. DSDP, 15:Washington (U.S. Govt. Printing Office), 553-615.

Sachs, J. B., 1970. Calcareous nannofossils of the Aftonian shale, Louisiana continental shelf [Ph.D. dissert.]. Tulane University, New Orleans.

Sachs, J. B., and Skinner, H. C., 1973. Calcareous nannofossils and late Pliocene-early Pleistocene biostratigraphy, Louisiana continental shelf. Tulane Stud. Geol. Paleontol., 10(3):113-162.

Smith, L. A., and Beard, J. H., 1973. The Late Neogene of the Gulf of Mexico. In Worzel, J. L., Bryant, W., et al., Init. Repts. DSDP, 10:Washington (U.S. Govt. Printing Office), 643-677.

Steinmetz, J. C., 1983a. Bibliography and taxa of calcareous nannoplankton. Int. Nannoplankton Assoc. Newsl., 5:4-13.

1983b. Bibliography and taxa of calcareous nannoplankton. Int. Nannoplankton Assoc. Newsl., 5:29-49. 1984. Bibliography and taxa of calcareous nannoplankton. Int. Nannoplankton Assoc. Newsl., 6:6-37.

Thierstein, H. R., Geitzenauer, K., Molfino, B., and Shackleton. N. J., 1977. Global synchroneity of Late Quaternary coccolith datums: validation by oxygen isotopes. Geology, 5:400-404.

Walker, J. R., and Massingill, J. V., 1970. Slump features on the Mississippi Fan, northeastern Gulf of Mexico. Geol. Soc. Am. Bull., 81:3101-3108.

Williams, D., 1984. Correlation of Pleistocene marine sediments of the Gulf of Mexico and other basins using oxygen isotope stratigraphy. In Healy-Williams, N. (Ed.), Principles of Pleistocene Stratigraphy Applied to the Gulf of Mexico: Boston (IHRDC Press), pp. 65-118.

Date of Initial Receipt: 5 December 1984 Date of Acceptance: 1 July 1985 

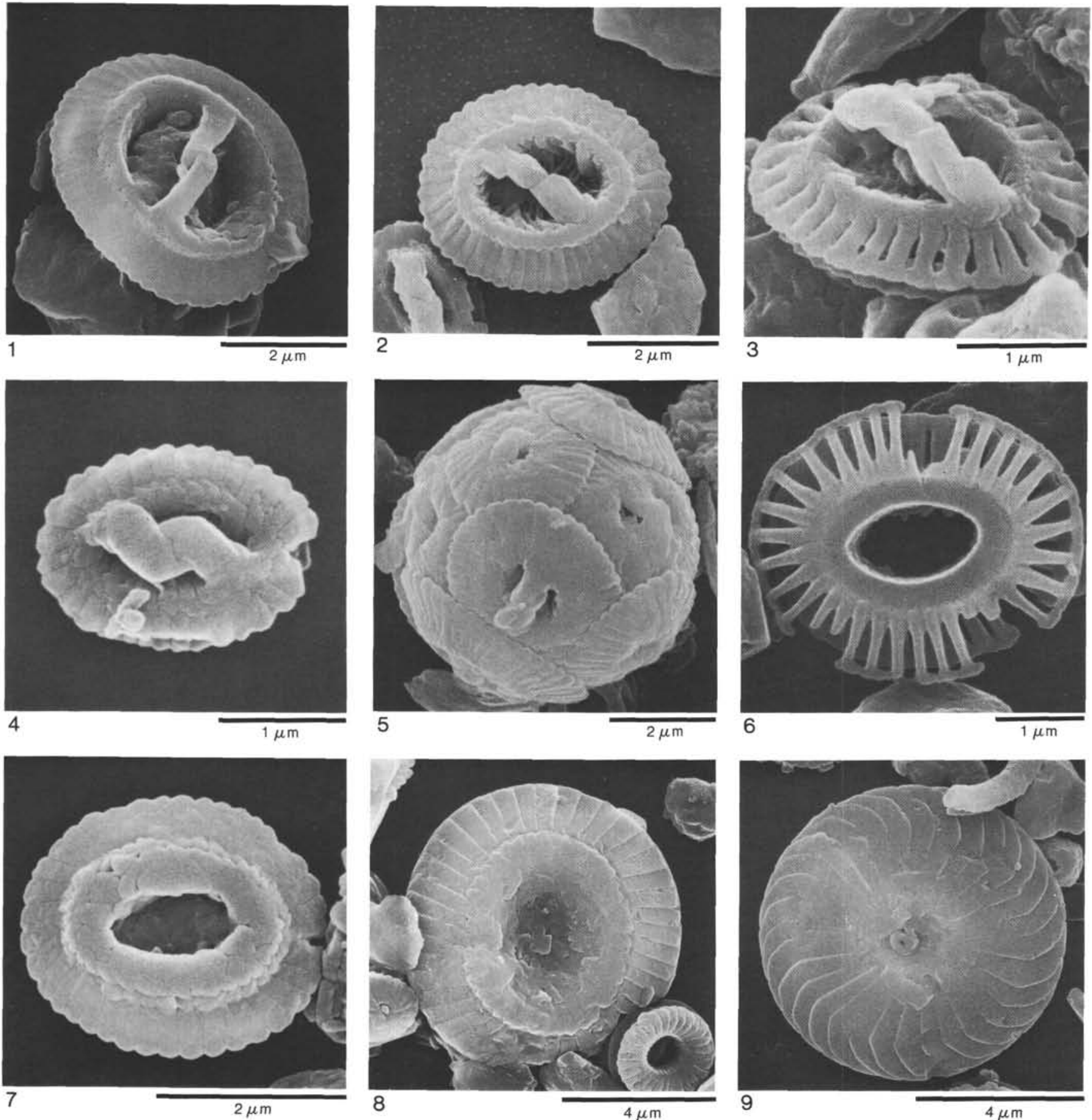

Plate 1. 1. Gephyrocapsa oceanica Kamptner, D, Sample 619-16,CC. 2. Gephyrocapsa caribbeanica Boudreaux and Hay, D, Sample 615-48,CC. 3. Gephyrocapsa protohuxleyi McIntyre, D, Sample 615-51,CC. 4. Gephyrocapsa sinuosa Hay and Beaudry, D, Sample 615-48,CC. 5. Gephyrocapsa spp. (small), coccosphere, Sample 615-48,CC. 6. Emiliania huxleyi (Lohmann) Hay and Mohler, D, Sample 615-51,CC. 7. Crenalithus doronicoides (Black and Barnes) Roth, D, Sample 615-48,CC. 8. Coccolithus pelagicus (Wallich) Schiller, D, Sample 615-51,CC. 9. Cyclococcolithus leptoporus (Murray and Blackman) Kamptner, D, Sample 615-51,CC. All figures are scanning electron micrographs. P = proximal view, $\mathrm{D}$ = distal view, $\mathrm{L}=$ lateral view. 


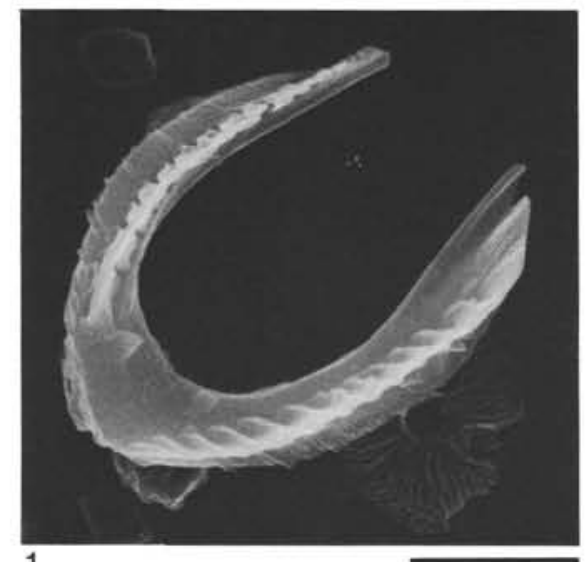

1
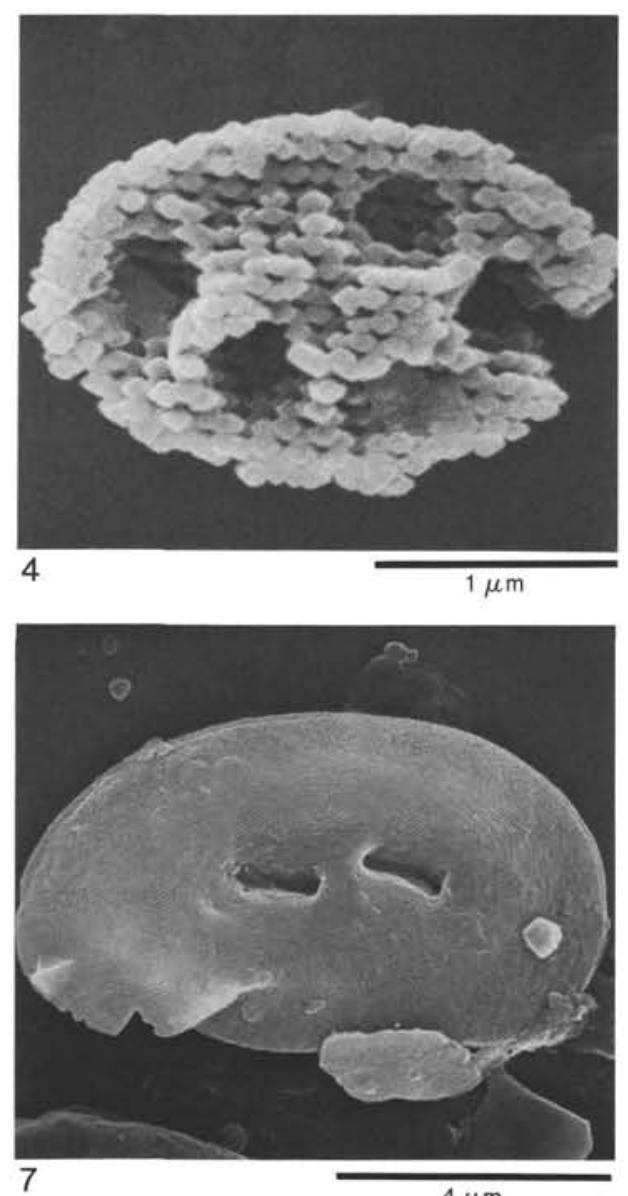

$4 \mu \mathrm{m}$

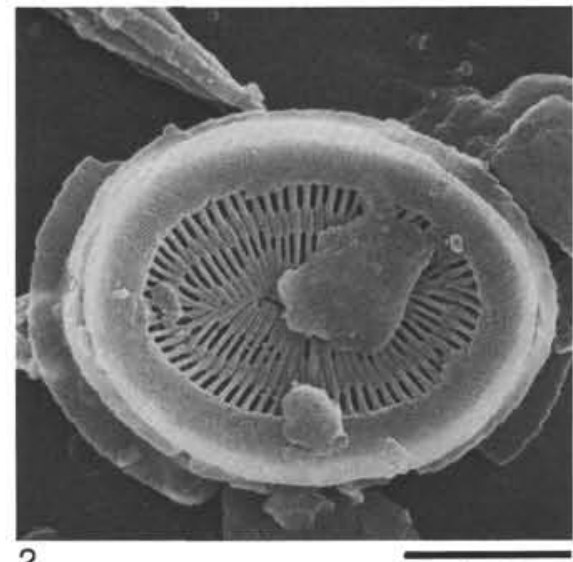

$2 \mu \mathrm{m}$

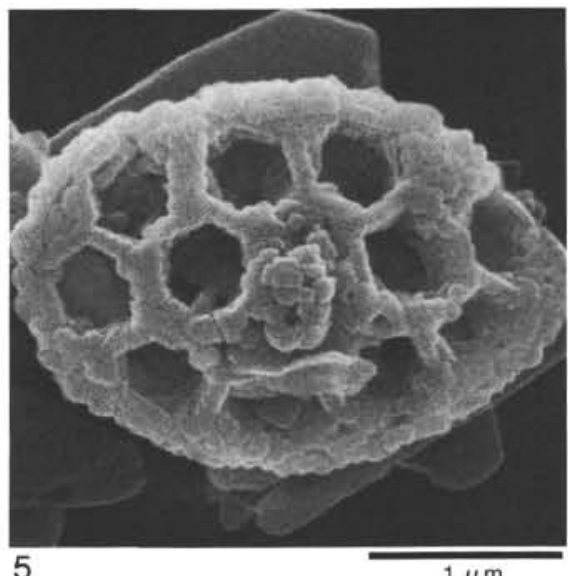

5

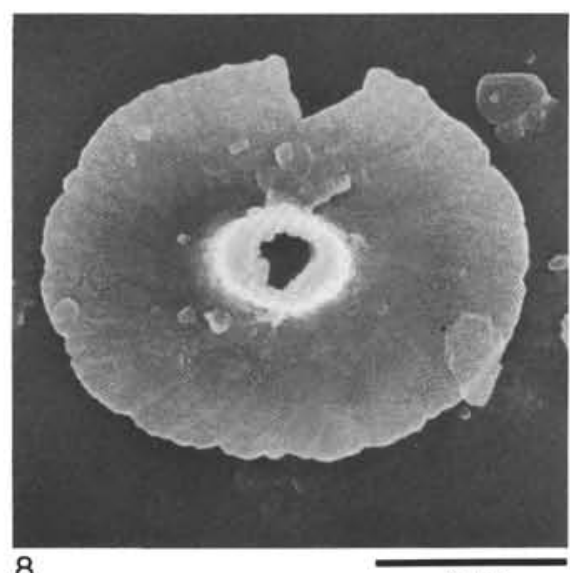

$2 \mu \mathrm{m}$
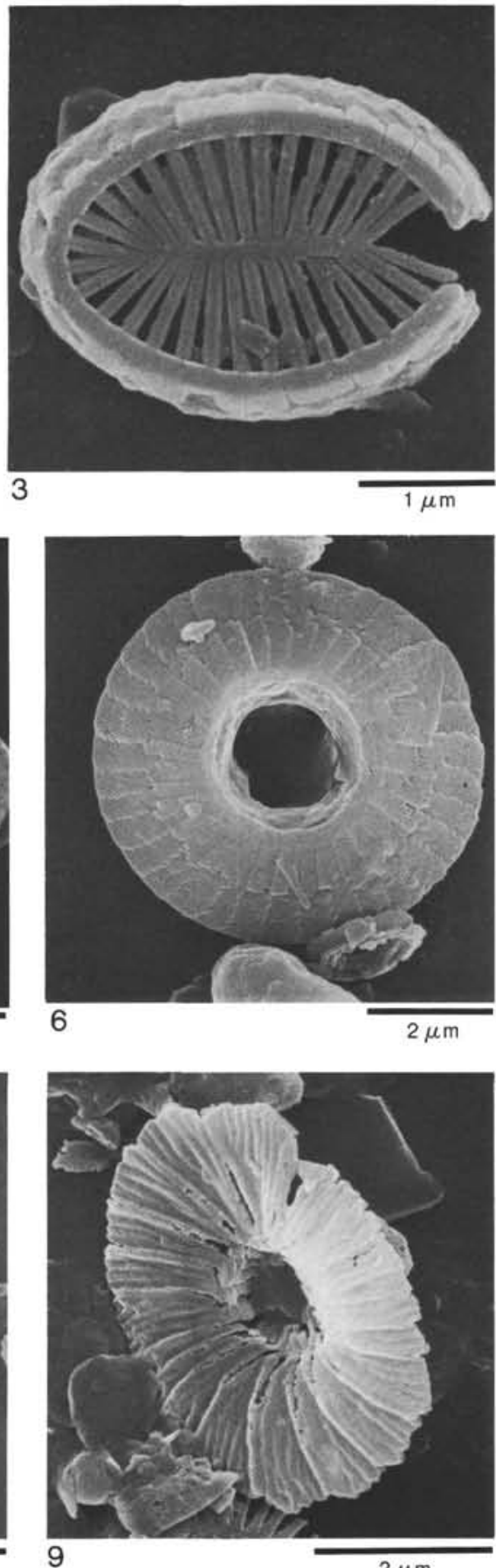

$2 \mu \mathrm{m}$

Plate 2. 1. Ceratolithus cristatus Kamptner, L, Sample 617A-1-1, 0-2 cm. 2. Syracosphaera pulchra Lohmann, P, Sample 619-16,CC. 3. Syracosphaera lamina Lecal-Schlauder s. ampl., P, Sample 619-16,CC. 4. Homozygosphaera wettsteinii (Kamptner) Halldal and Markali, P, Sample 619-16,CC. 5. Homozygosphaera schilleri (Kamptner) Okada and McIntyre, D, Sample 615-51-1, 41-42 cm. 6. Umbilicosphaera mirabilis Lohmann, D, Sample 619-16,CC. 7. Helicosphaera wallichii (Lohmann) Okada and McIntyre, D, Sample 619-16,CC. 8. Umbellosphaera sp. distal plate, P, Sample 619-16,CC. 9. Umbellosphaera tenuis (Kamptner) Paasche, D, Sample 617A-1-1, 0-2 cm. All figures are scanning electron micrographs. $\mathrm{P}=$ proximal view $\mathrm{D}=$ distal view, $\mathrm{L}=$ lateral view. 

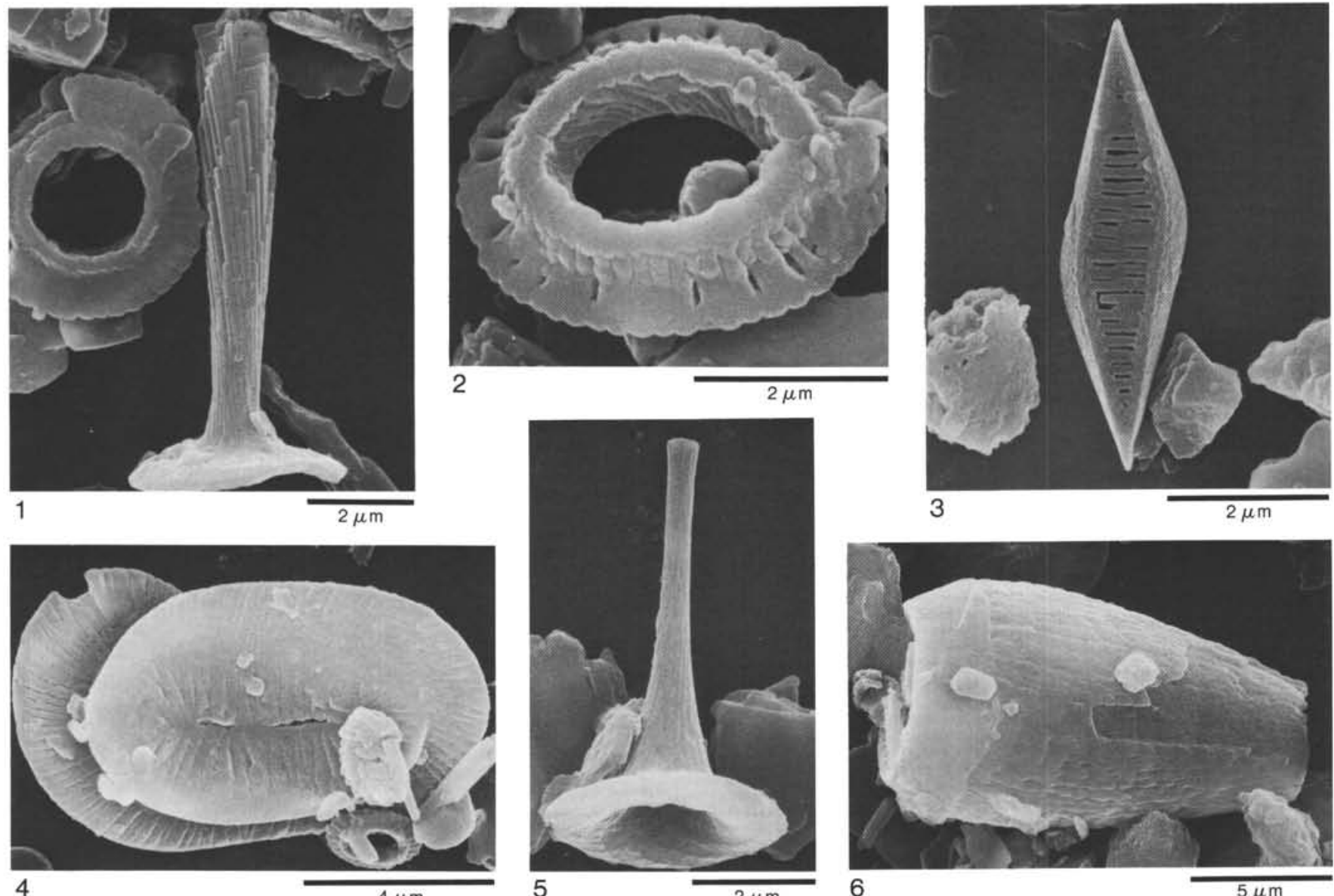

$4 \mu \mathrm{m}$

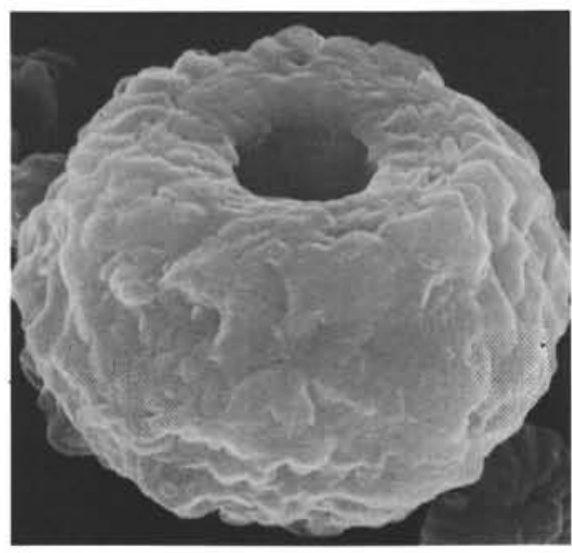

8

$2 \mu \mathrm{m}$

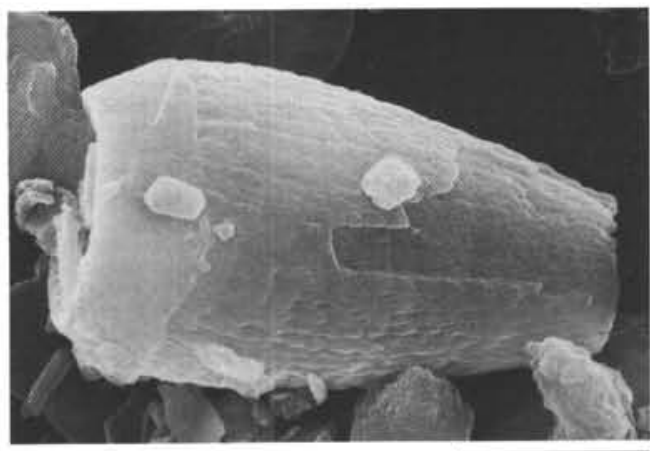

6

$5 \mu \mathrm{m}$

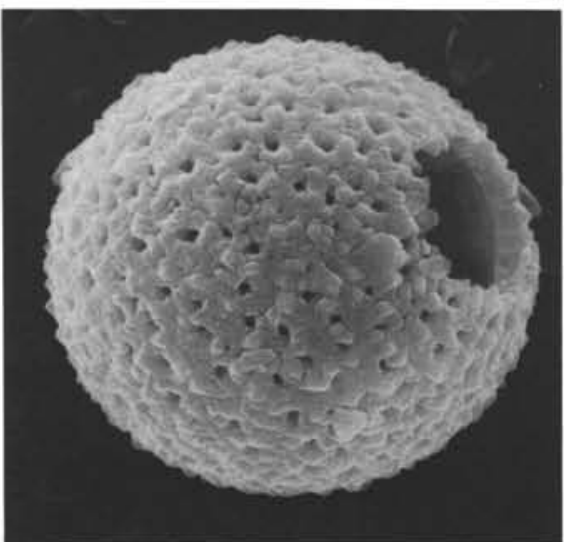

7

$4 \mu \mathrm{m}$

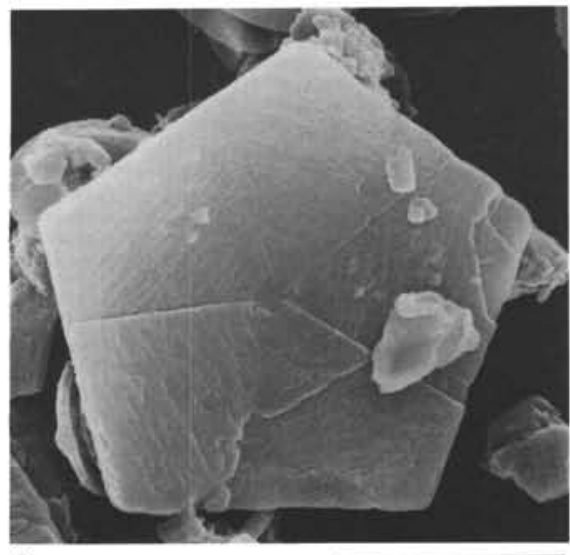

9

$4 \mu \mathrm{m}$

Plate 3. 1. Rhabdosphaera clavigera Murray and Blackman, L, Sample 615-48,CC. 2. Pseudoemiliania lacunosa (Kamptner) Gartner, D, Sample 615-48,CC. 3. Scapholithus fossilis Deflandre, P, Sample 619-16,CC. 4. Helicosphaera carteri (Wallich) Kamptner, P, Sample 615-48,CC. 5. Discosphaera tubifera (Murray and Blackman) Kamptner, L, Sample 619-16,CC. 6. Scyphosphaera sp., L, Sample 615-49-4, 117-118 cm. 7. Thoracosphaera heimii (Lohmann) Kamptner, L, Sample 619-16,CC. 8. Thoracosphaera sp., L, Sample 615-48,CC. 9. Braarudosphaera bigelowii (Gran and Braarud) Deflandre, P, Sample 615-48,CC. All figures are scanning electron micrographs. $\mathrm{P}=$ proximal view, D $=$ distal view, $\mathrm{L}=$ lateral view. 

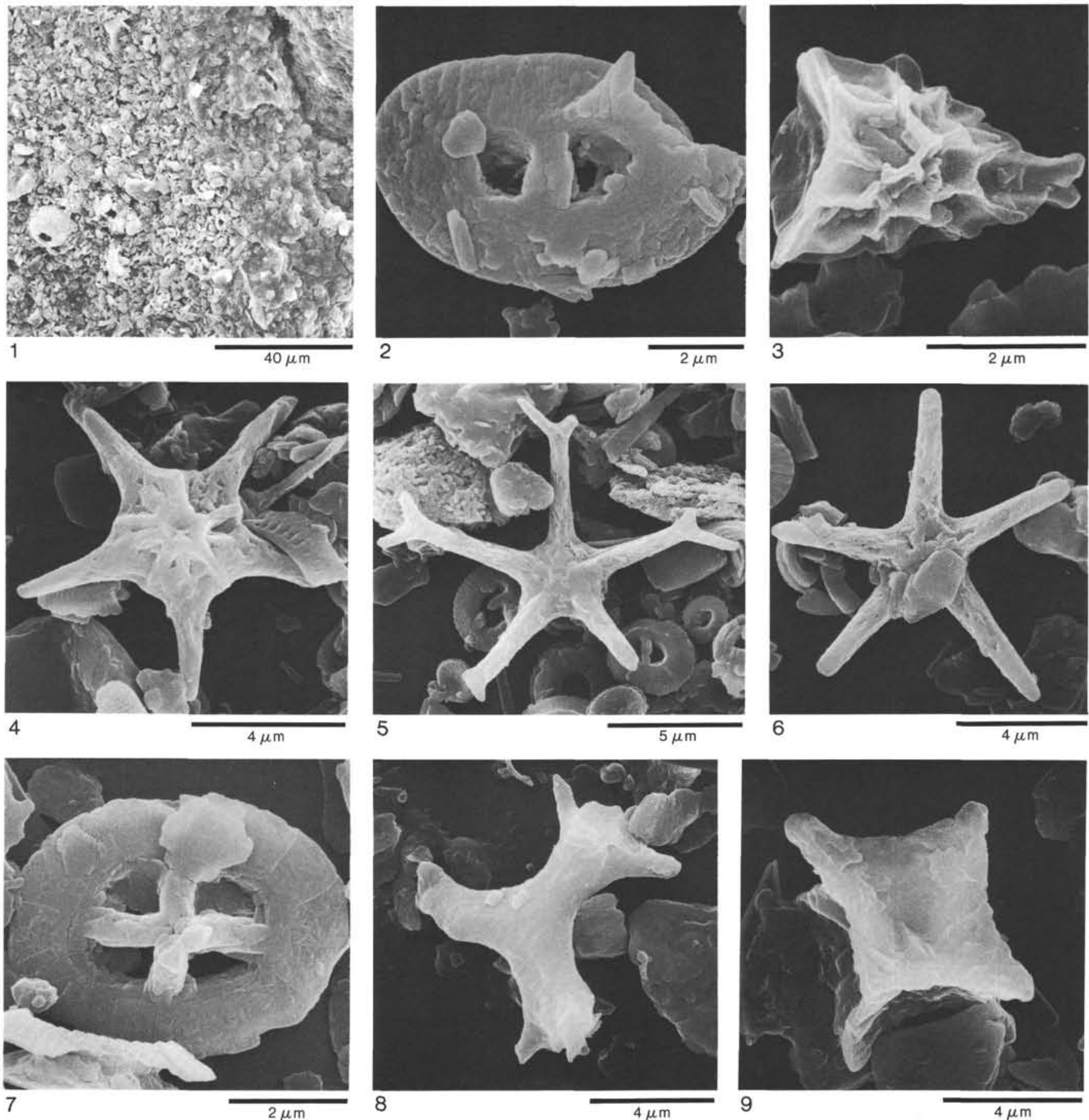

Plate 4. 1. Boundary between reworked calcareous clast with abundant calcareous nannofossils (left side) and mud matrix (right side), Sample 6184-4, 120-126 cm. 2. Helicosphaera sellii (Bukry and Bramlette) Jafar and Martini, D, Sample 615-48,CC. 3. Sphenolithus abies Deflandre, L, Sample 615-51,CC. 4. Discoaster berggrenii Bukry, D, Sample 615-48,CC. 5. Discoaster pentaradiatus Tan, P, Sample 615-48,CC. 6. Discoaster asymmetricus Gartner, P, Sample 615-48,CC. 7. Prediscosphaera spinosa (Bramlette and Martini) Gartner, D, Sample 619-16,CC. 8. Marthasterites furcatus (Deflandre and Fert) Deflandre, L, Sample 619-16,CC. 9. Micula decussata concava (Stradner) Bukry, L, Sample 619$16, \mathrm{CC}$. All figures are scanning electron micrographs. $\mathrm{P}=$ proximal view, $\mathrm{D}=$ distal view, $\mathrm{L}=$ lateral view. 